University of Louisville

ThinkIR: The University of Louisville's Institutional Repository

Electronic Theses and Dissertations

8-2004

\title{
Modeling real gases and liquids using a modified van der Waals equation of state.
}

Andrew James Sexton 1981-

University of Louisville

Follow this and additional works at: https://ir.library.louisville.edu/etd

\section{Recommended Citation}

Sexton, Andrew James 1981-, "Modeling real gases and liquids using a modified van der Waals equation of state." (2004). Electronic Theses and Dissertations. Paper 1301.

https://doi.org/10.18297/etd/1301

This Master's Thesis is brought to you for free and open access by ThinkIR: The University of Louisville's Institutional Repository. It has been accepted for inclusion in Electronic Theses and Dissertations by an authorized administrator of ThinkIR: The University of Louisville's Institutional Repository. This title appears here courtesy of the author, who has retained all other copyrights. For more information, please contact thinkir@louisville.edu. 
MODELING REAL GASES AND LIQUIDS USING A MODIFIED VAN DER WAALS EQUATION OF STATE

By

Andrew James Sexton

B.S., University of Louisville, 2003

\author{
A Thesis \\ Submitted to the Faculty of the \\ University of Louisville \\ Speed Scientific School \\ as Partial Fulfillment of the Requirements \\ for the Professional Degree
}

MASTER OF ENGINEERING

Department of Chemical Engineering

August 2004 



\section{MODELING REAL GASES AND LIQUIDS USING A MODIFIED VAN DER WAALS EQUATION OF STATE}

Sumitted by:

Andrew James Sexton

A Thesis Approved on

$\underline{\text { June } 10,2004}$

(Date)

by the Following Reading and Examination Committee:

Dermot J. Collins, Thesis Director

Patricia A. Ralston

John F. Naber 


\section{ACKNOWLEDGEMENTS}

First and foremost, I want to thank my parents, Joe and Carol Sexton, for their endless love and support throughout my tenure at the University of Louisville. Their guidance, which included weekly letters of encouragement, has had an immeasurable influence in every aspect of my life.

Next, I want to recognize all of the friendships I have established at the University of Louisville. I thank my classmates at Speed School for driving me to achieve my full potential and never settle. I wish them all the best in their future endeavors. I also thank friends outside of Speed School, who kept me modest and helped me discover my true identity.

Lastly, I would like to thank those who facilitated the completion of this thesis. Thanks to Dr. Ralston and Dr. Naber for serving on my thesis committee. Special thanks are reserved for Dr. Collins for serving as my thesis advisor. His candidness and semiunique wit provided me a refreshing and much-needed outlook on life's travails over the past five years. I consider him a colleague and a very good friend; a simple thanks doesn't do his effect on my life justice. 


\begin{abstract}
Equations of state attempt to describe the relationship between temperature $(\mathrm{T})$, pressure $(\mathrm{P})$, and molar volume $(\mathrm{v})$ for a given substance or mixture of substances. The ideal gas law is the simplest form of an equation of state. An ideal gas can be considered as a large quantity of small molecules that have no friction, no attractive or repulsive forces. The ideal gas law is a reasonable approximation at low pressures and high temperatures, but not at higher pressures and lower temperatures. Thus, better methods for predicting real gas behavior have been continuously introduced over the past 200 years.
\end{abstract}

Another approximation is to assume that gas atoms and molecules behave as hard spheres. These spheres are incompressible and only repulsive forces are significant at the moment of collision. A recent modification made to the van der Waals equation of state (VDW) incorporates the hard sphere model, giving better representation of the van der Waals parameters over a broader temperature and pressure range.

The efficacy of this modified van der Waals equation of state was assessed for six previously researched compounds -ethane, propane, $n$-butane, $n$-pentane, argon and water. Physical property charts (specifically molar volume and molar enthalpy charts) were developed for these substances using the original VDW and modified VDW, as well as the Redlich-Kwong (RK) and Redlich-Kwong-Soave (RKS) equations of state. Results for molar volume revealed that for the four hydrocarbons, the modified VDW compared best with the given experimental data, but not for argon and water. Results for molar enthalpy showed the original VDW compared more favorably with experimental data than the original VDW, but not as well as the RK equation of state. 


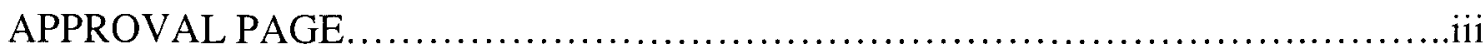

ACKNOWLEDGEMENTS .......................................................

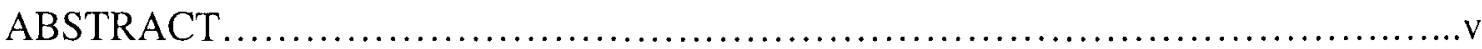

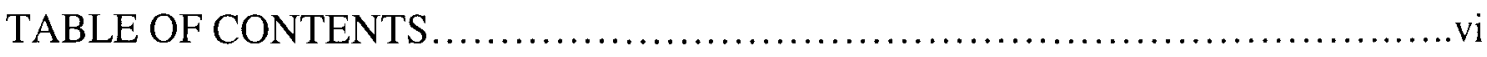

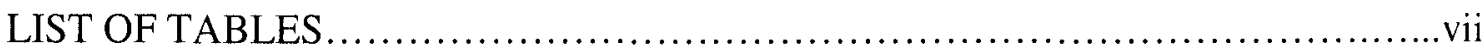

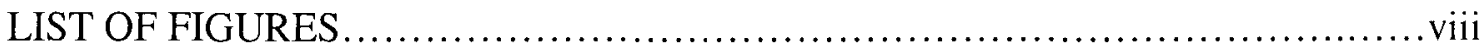

NOMENCLATURE ............................................................

I. INTRODUCTION ................................................

II. LITERATURE REVIEW ...........................................

A. Evolution of Equations of State.............................5

B. The van der Waals Equation of State and Some Simple Modifications..................................................9

C. Thermodynamic Properties from Equations of State.............16

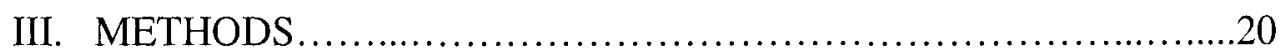

IV. RESULTS AND DISCUSSION ..................................30

V. CONCLUSIONS .............................................. 43

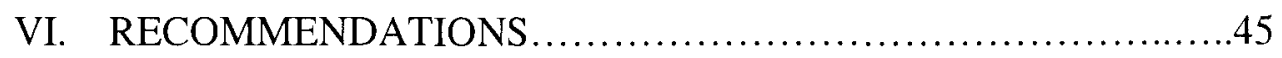

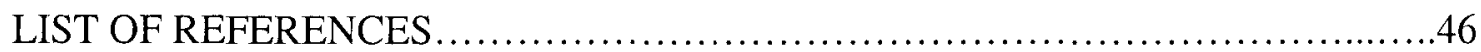

APPENDIX I. PRESSURE-MOLAR VOLUME GRAPHS .......................47

APPENDIX II. PRESSURE-MOLAR ENTHALPY GRAPHS ......................60

APPENDIX III. RESIDUAL ENTHALPY CALCULATIONS ........................70

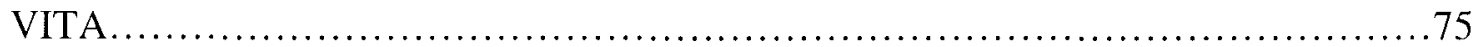




\section{LIST OF TABLES}

Table I - Equations of State.

Table II - Modifications to van der Waals Parameters 10

Table III - Comparison of van der Waals $b$-Parameters.............................14

Table IV - Modified van der Waals $a$-Parameters......................................16

Table V - Residual Enthalpy for Equations of State...............................19

Table VI - Equilibrium Vapor and Liquid Molar Volumes Using the van der Waals Equation of State

Table VII - Equations of State in Vapor Molar Volume Explicit Form.................23

Table VIII - Equations of State in Liquid Molar Volume Explicit Form ................24

Table IX - Equilibrium Vapor and Liquid Molar Enthalpies for Ethane Using the Original van der Waals Equation of State..................................26

Table X - Effect of $a$-Parameter on Ethane Residual Enthalpy........................40

Table XI - Effect of $a$-Parameter on Ethane Residual Enthalpy ......................41 


\section{LIST OF FIGURES}

Figure 1 - The Phase Diagram .....................................................

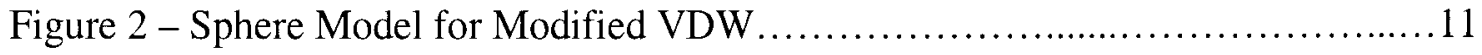

Figure 3 - Theoretical Pressure-Volume Diagram.....................................21

Figure 4 - Theoretical Log Pressure-Enthalpy Diagram ................................21

Figure 5 - Vapor and Liquid Molar Volumes of Argon Using the Reidlich-Kwong-Soave

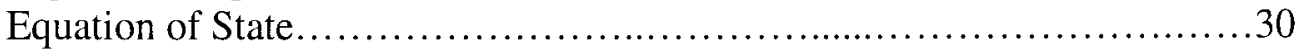

Figure 6 - Vapor and Liquid Molar Enthalpies of Argon Using the Reidlich-Kwong Equation of State ................................................... 31

Figure 7 - Effect of Pressure on Argon Molar Volume ..................................33

Figure 8 - Effect of Pressure on Ethane Molar Volume...............................33

Figure 9 - Effect of Pressure on Propane Molar Volume ................................34

Figure 10 - Effect of Pressure on $n$-Butane Molar Volume .............................34

Figure 11 - Effect of Pressure on $n$-Pentane Molar Volume ...........................35

Figure 12 - Effect of Pressure on Water Molar Volume ..............................35

Figure 13 - Effect of Pressure on Argon Molar Enthalpy ...........................37

Figure 14 - Effect of Pressure on Ethane Molar Enthalpy ............................37

Figure 15 - Effect of Pressure on Propane Molar Enthalpy ...........................38

Figure 16 - Effect of Pressure on n-Butane Molar Enthalpy ..........................38

Figure 17 - Effect of Pressure on n-Pentane Molar Enthalpy ..........................39

Figure 18 - Effect of Pressure on Water Molar Enthalpy ...........................39

Figure 19 - Vapor and Liquid Molar Volumes of Argon Using the Reidlich-Kwong Equation of State .................................................48

Figure 20 - Vapor and Liquid Molar Volumes of Argon Using the Original van der Waals Equation of State 
Figure 21- Vapor and Liquid Molar Volumes of Argon Using the Modified van der Waals Equation of State.....

Figure 22 - Vapor and Liquid Molar Volumes of Ethane Using the Reidlich-KwongSoave Equation of State.

Figure 23 - Vapor and Liquid Molar Volumes of Ethane Using the Reidlich-Kwong Equation of State

Figure 24 - Vapor and Liquid Molar Volumes of Ethane Using the Original van der Waals Equation of State

Figure 25 - Vapor and Liquid Molar Volumes of Ethane Using the Modified van der Waals Equation of State

Figure 26 - Vapor and Liquid Molar Volumes of Propane Using the Reidlich-KwongSoave Equation of State

Figure 27 - Vapor and Liquid Molar Volumes of Propane Using the Reidlich-Kwong Equation of State

Figure 28 - Vapor and Liquid Molar Volumes of Propane Using the Original van der Waals Equation of State

Figure 29 - Vapor and Liquid Molar Volumes of Propane Using the Modified van der Waals Equation of State

Figure 30 - Vapor and Liquid Molar Volumes of $n$-Butane Using the Reidlich-KwongSoave Equation of State

Figure 31 - Vapor and Liquid Molar Volumes of $n$-Butane Using the Reidlich-Kwong Equation of State .54

Figure 32 - Vapor and Liquid Molar Volumes of $n$-Butane Using the Original van der Waals Equation of State

Figure 33 - Vapor and Liquid Molar Volumes of $n$-Butane Using the Modified van der Waals Equation of State.

Figure 34 - Vapor and Liquid Molar Volumes of $n$-Pentane Using the Reidlich-KwongSoave Equation of State

Figure 35 - Vapor and Liquid Molar Volumes of $n$-Pentane Using the Reidlich-Kwong Equation of State 
Figure 36 - Vapor and Liquid Molar Volumes of $n$-Pentane Using the Original van der Waals Equation of State

Figure 37 - Vapor and Liquid Molar Volumes of $n$-Pentane Using the Modified van der Waals Equation of State.

Figure 38 - Vapor and Liquid Molar Volumes of Water Using the Reidlich-KwongSoave Equation of State .58

Figure 39 - Vapor and Liquid Molar Volumes of Water Using the Reidlich-Kwong Equation of State .58

Figure 40 - Vapor and Liquid Molar Volumes of Water Using the Original van der Waals Equation of State

Figure 41 - Vapor and Liquid Molar Volumes of Water Using the Modified van der Waals Equation of State

Figure 42 - Vapor and Liquid Molar Enthalpies of Argon Using the Original van der

Waals Equation of State

Figure 43 - Vapor and Liquid Molar Enthalpies of Argon Using the Modified van der Waals Equation of State

Figure 44 - Vapor and Liquid Molar Enthalpies of Ethane Using the Reidlich-Kwong Equation of State

Figure 45 - Vapor and Liquid Molar Enthalpies of Ethane Using the Original van der Waals Equation of State .63

Figure 46 - Vapor and Liquid Molar Enthalpies of Ethane Using the Modified van der Waals Equation of State .63

Figure 47 - Vapor and Liquid Molar Enthalpies of Propane Using the Reidlich-Kwong Equation of State .64

Figure 48 - Vapor and Liquid Molar Enthalpies of Propane Using the Original van der Waals Equation of State .64

Figure 49 - Vapor and Liquid Molar Enthalpies of Propane Using the Modified van der Waals Equation of State

Figure 50 - Vapor and Liquid Molar Enthalpies of $n$-Butane Using the Reidlich-Kwong Equation of State 
Figure 51 - Vapor and Liquid Molar Enthalpies of $n$-Butane Using the Original van der Waals Equation of State

Figure 52 - Vapor and Liquid Molar Enthalpies of $n$-Butane Using the Modified van der Waals Equation of State

Figure 53 - Vapor and Liquid Molar Enthalpies of $n$-Pentane Using the Reidlich-Kwong Equation of State

Figure 54 - Vapor and Liquid Molar Enthalpies of $n$-Pentane Using the Original van der Waals Equation of State

Figure 55 - Vapor and Liquid Molar Enthalpies of $n$-Pentane Using the Modified van der Waals Equation of State

Figure 56 - Vapor and Liquid Molar Enthalpies of Water Using the Reidlich-Kwong Equation of State ....................................................68

Figure 57 - Vapor and Liquid Molar Enthalpies of Water Using the Original van der Waals Equation of State

Figure 58 - Vapor and Liquid Molar Enthalpies of Water Using the Modified van der Waals Equation of State 

$\mathrm{A}=\quad$ Antoine equation coefficient
$a=$ original van der Waals $a$-parameter
$a_{0}=$ modified van der Waals $a$-parameter at critical conditions
$a_{H S}=$ modified van der Waals $a$-parameter
$a_{I} \quad=\quad$ modified van der Waals constant
$a_{2}=$ modified van der Waals constant
$\mathrm{B}=$ Antoine equation coefficient
$b \quad=\quad$ original van der Waals $b$-parameter
$b_{a v}=$ actual atomic molar volume
$b_{H S}=$ modified van der Waals $b$-parameter
$b_{0}=$ modified van der Waals constant
$\mathrm{C}=$ Antoine equation coefficient
$\mathrm{C}_{\mathrm{p}}^{\mathrm{ig}}=\quad$ ideal gas heat capacity
$\mathrm{G}=$ Gibbs energy
$\mathrm{G}^{\mathrm{ig}}=$ Gibbs energy for ideal gas
$\mathrm{G}^{\mathrm{R}} \quad=\quad$ residual Gibbs energy
$\mathrm{H}_{0}{ }^{\mathrm{ig}}=$ standard-state enthalpy for ideal gas
$\mathrm{H}^{\mathrm{R}} \quad=\quad$ residual enthalpy
$\mathrm{N}_{\mathrm{A}}=$ Avagadro's number
$\sigma=$ Lennard-Jones parameter
$\mathrm{P}=$ pressure 


$$
\begin{array}{lll}
\mathrm{P}_{\mathrm{c}} & = & \text { critical pressure } \\
\mathrm{P}_{\mathrm{o}} & = & \text { standard-state pressure } \\
\mathrm{P}^{\mathrm{sat}} & = & \text { saturation pressure } \\
\mathrm{R} & = & \text { universal gas constant } \\
\mathrm{S} & = & \text { entropy } \\
\mathrm{T} & = & \text { temperature } \\
\mathrm{T}_{\mathrm{c}} & = & \text { critical temperature } \\
\mathrm{T}_{\mathrm{o}} & = & \text { standard-state temperature } \\
\mathrm{T}_{\mathrm{r}} & = & \text { reduced temperature } \\
\mathrm{U} & = & \text { internal energy } \\
\mathrm{v} & = & \text { molar volume } \\
\mathrm{v}_{\mathrm{a}} & = & \text { atomic molar volume } \\
v_{\mathrm{eff}} & = & \text { effective molar volume } \\
v_{\mathrm{g}} & = & \text { vapor molar volume } \\
v_{1} & = & \text { liquid molar volume } \\
\omega & = & \text { acentric factor } \\
\mathrm{Z} & &
\end{array}
$$




\section{INTRODUCTION}

All current specialized studies pertaining to the behavior of gaseous compounds can be traced back to one individual, Jacques Charles. In 1787, Charles, a French physicist and chemist, demonstrated the volume of a dry gas varies directly with temperature in a constant pressure environment. Other early innovators, whose work followed Charles', performed experiments at temperatures and pressures such that distance between molecules was great enough to neglect the effect of intermolecular forces and friction. This type of gas, composed of a large quantity of small molecules that have no friction, no attractive or repulsive forces, was defined to be an ideal gas. The equation that describes the relationship between temperature, pressure, and volume under these conditions is known as the ideal gas law (Himmelblau, 2000).

The ideal gas law is the simplest form of an equation of state. Equations of state attempt to describe the relationship between temperature $(\mathrm{T})$, pressure $(\mathrm{P})$, and molar volume $(v)$ for a given substance or mixture of substances. The ideal gas law states the following relationship (Smith et al., 2002)

$$
P v=R T
$$

where $\mathrm{R}$ is the universal gas constant. The compressibility factor $(\mathrm{Z})$ is a dimensionless quantity relating the behavior of a real gas to that of an ideal gas expressed by (Smith et al., 2002): 
Specifically, the compressibility factor of an ideal gas is one. Although the ideal gas law is a reasonable model for predicting behavior of gases at low pressures and high temperatures (when atoms are spaced far apart and intermolecular forces are negligible), it becomes increasingly inaccurate at higher pressures and lower temperatures (when intermolecular forces are appreciable). Thus, better methods for predicting the behavior of real gases were needed. One early model was the van der Waals model for a fluid composed of particles of non-zero size and a pairwise interparticle force. The van der Waals equation was attractive because it is a relatively simple expression that predicts the formation of a liquid phase where applicable. Over the years, improved equations of state have followed the van der Waals equation. They include the Virial equation, Reidlich-Kwong equation of state, Reidlich-Kwong-Soave equation of state, and PengRobinson equation of state. While each of these equations may predict results more accurately, they also become increasingly more complex to analyze. They involve the computation of reduced parameters and compressibility factors (Himmelblau, 2000).

The original van der Waals equation of state is represented by the expression (Smith et al., 2002):

$$
P=R T /(v-b)-a / v^{2}
$$

Parameters $b$ and $a$ are constants whose values are determined from the measured critical pressure value $\mathrm{P}_{\mathrm{c}}$ and the critical temperature value $\mathrm{T}_{\mathrm{c}}$. The constant $b$ accounts for the atomic volume of the gaseous molecules, while parameter $a$ is a molar enhancement factor that accounts for attractive interactions between the molecules. Since the critical 
isotherm exhibits a horizontal inflection at the critical point, we may impose the mathematical conditions (Smith et al., 2002)

$$
(\partial P / \partial v)_{T c}=\left(\partial^{2} P / \partial v^{2}\right)_{T C}=0
$$

where the subscript "c" denotes the critical point. Differentiation of the pressure-explicit form of the VDW equation of state yields expressions for the above derivatives, which may be set equal to zero. Using algebraic manipulation, it can be shown that (Smith et al., 2002):

$$
\begin{gathered}
a=(27 / 64) * R^{2} T_{c}^{2} / P_{c} \\
b=(1 / 8) * R T_{c} / P_{c}
\end{gathered}
$$

According to these equations, the parameters $a$ and $b$ are constant values. However, it is well known that these parameters are not constant, varying somewhat with temperature and pressure (McNeill and Collins, 2004).

The purpose of this research is to investigate the efficacy of a modified van der Waals equation of state, based on the assumption that the behavior of gas atoms can be approximated by hard spheres. That is, the value of $b$ is derived from hard sphere behavior. Also, an empirical algebraic equation is incorporated to account for variations in $a$ with respect to temperature. Thus, physical property charts (specifically molar volume and molar enthalpy charts) will be developed by calculating the residual properties for six compounds (argon, ethane, propane, $n$-butane, $n$-pentane, and water). 
These residual property values will then be compared and contrasted with values obtained using the Reidlich-Kwong-Soave, Reidlich-Kwong, and original van der Waals equations of state. The goal of this thesis is to revise an existing simplified model and achieve a precision normally attained by more complex equations of state. 


\section{LITERATURE REVIEW}

\section{A. Evolution of Equations of State}

As described in Chapter I, equations of state relate the specific volume ( $v$ ) of a substance as a function of temperature $(\mathrm{T})$ and pressure $(\mathrm{P})$ via empirical relationships. TABLE I lists some of the more commonly used equations of state in addition to the Van der Waals equation of state (Himmelblau, 2000):

\section{TABLE I}

EQUATIONS OF STATE (Himmelblau, 2000)

\begin{tabular}{|c|c|}
\hline van der Waals & $\left(P+a / v^{2}\right)^{*}(v-b)=R T$ \\
\hline & $a=(27 / 64) *\left(R^{2 *} T_{c}^{2} / P_{c}\right)$ \\
\hline & $\mathrm{b}=(1 / 8)^{*}\left(\mathrm{R}^{*} \mathrm{~T}_{\mathrm{c}} / \mathrm{P}_{\mathrm{c}}\right)$ \\
\hline \multirow[t]{3}{*}{ Reidlich-Kwong } & {$\left[P+a / T^{1 / 2 *} v^{*}(v+b)\right]^{*}(v-b)=R T$} \\
\hline & $a=0.42748 *\left(R^{2 *} T_{c}^{2.5} / P_{c}\right)$ \\
\hline & $\mathrm{b}=0.08664 *\left(\mathrm{R}^{\star} \mathrm{T}_{\mathrm{c}} / \mathrm{P}_{\mathrm{c}}\right)$ \\
\hline \multirow[t]{6}{*}{ Reidlich-Kwong-Soave } & $P=(R T / v-b)-\left(a \alpha / v^{*}(v+b)\right)$ \\
\hline & $a=0.42748^{*}\left(R^{2} T_{c}^{2} / P_{c}\right)$ \\
\hline & $\mathrm{b}=0.08664{ }^{*}\left(\mathrm{R}^{*} \mathrm{~T}_{\mathrm{c}} / \mathrm{P}_{\mathrm{c}}\right)$ \\
\hline & $a=\left[1+K^{*}\left(1-T_{r}^{1 / 2}\right)\right]^{2}$ \\
\hline & $\kappa=0.480+1.574^{*} \omega-0.176^{*} \omega^{2}$ \\
\hline & $\omega=$ acentric factor \\
\hline \multirow[t]{6}{*}{ Peng-Robinson } & $P=(R T / v-b)-\left(a \alpha / v^{*}(v-b)+b^{*}(v-b)\right)$ \\
\hline & $a=0.45724 *\left(R^{2} T_{c}^{2} / P_{c}\right)$ \\
\hline & $\mathrm{b}=0.07780^{*}\left(\mathrm{R}^{*} \mathrm{~T}_{\mathrm{c}} / \mathrm{P}_{\mathrm{c}}\right)$ \\
\hline & $a=\left[1+K^{*}\left(1-T_{r}^{1 / 2}\right)\right]^{2}$ \\
\hline & $\kappa=0.37464+1.54226^{*} \omega-0.26992^{*} \omega \omega^{2}$ \\
\hline & $\omega=$ acentric factor \\
\hline Kammerlingh-Onnes & $\mathrm{PV}=\mathrm{RT}{ }^{*}\left(1+\mathrm{B} / \mathrm{N}+\mathrm{C} / \mathrm{V}^{2}+\ldots\right)$ \\
\hline Holborn & $P V=R T^{*}\left(1+B^{\prime} P+C^{\prime} P^{2}+\ldots\right)$ \\
\hline
\end{tabular}


All of the equations in TABLE I are listed in general form. Each of the equations of state can be rearranged and presented in a volume-explicit form. Two-constant forms of the equation of state include the Reidlich-Kwong, Reidlich-Kwong-Soave, and PengRobinson equations of state. These equations often do a better job of predicting the P-vT properties of particular substances, but the calculations involve more intensive methods of determining the constants; additionally, they involve the input of extra constants (acentric factor, for example). The equations illustrated by Kammerlingh-Onnes and Holborn are known as virial forms of the equation of state. They are essentially infinite power series based on either $(1 / \mathrm{v})$, the reciprocal of the molar volume, or P, total pressure. The variables $\mathrm{B}, \mathrm{C}$, and $\mathrm{D}$, etc., are temperature-dependent quantities known as virial coefficients. The coefficients $\mathrm{B}$ and $\mathrm{C}$ are calculated from statistical thermodynamics (Prausnitz et al., 1999). It is important to note that all of these equations reduce to the ideal gas law (equation 1) at low pressures (Himmelblau, 2000).

Despite their shortcomings, equations of state are important for a number of reasons. They allow for an ordered summary of an intense amount of experimental data and permit for accurate interpolation between the experimental data points. Furthermore, expressing the P-v-T relationships as continuous functions facilitates the calculation of physical properties using differentiation and integration. Most important, equations of state provide a starting point in the determination of the thermodynamic properties of mixtures (Himmelblau, 2000).

With the exception of the virial forms of the equation of state, the law of the corresponding states provides an explanation for the above equations. Early experimenters found that at the critical point all substances are in approximately the same 
state of molecular dispersion (Himmelblau, 2000). In other words, the compressibility factor (Z), for different fluids, exhibits similar behavior when related to its critical state, which is defined for the gas-liquid transition as the set of physical conditions at which the density and other properties of the liquid and vapor become identical. For pure components, the critical state is the highest temperature at which liquid and vapor can exist in equilibrium (Smith et al., 2002). The physical meaning of the critical temperature and critical pressure of a real gas is exhibited in FIGURE 1.

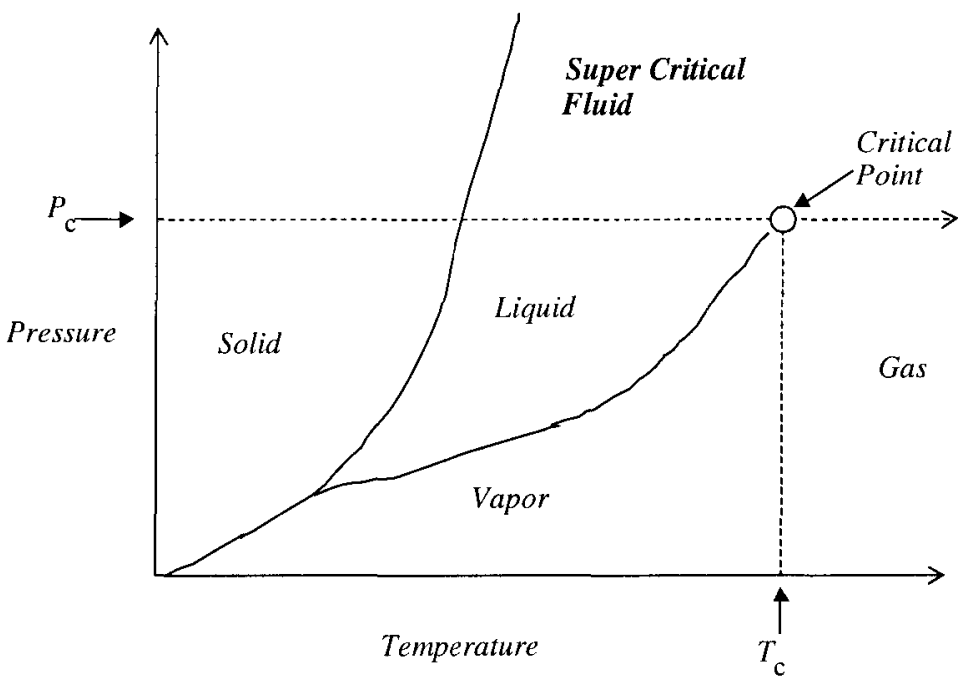

FIGURE 1 - The Phase Diagram

The critical state is determined using a set of terms known as the reduced conditions. These are conditions of temperature, pressure, and molar volume normalized to the values at the critical point. This led to the development of the two-parameter theorem of corresponding states as suggested by van der Waals. It states that all fluids, when 
compared at the same reduced temperature and reduced pressure, have approximately the same compressibility factor, and all deviate from ideal-gas behavior to about the same degree (Smith et al., 2002). Simply put, any substance would have the same reduced molar volume at the same reduced temperature and pressure. The compressibility factor is a dimensionless quantity relating the behavior of a real gas to that of an ideal gas. As noted in Chapter I, the compressibility factor of an ideal gas is one.

While the two-parameter theory of corresponding states provided early theoreticians with an explanation for the behavior of simple fluids, deviations were observed for more complex fluids. Subsequent research, coupled with advent of computers and other advanced machines, allowed for a more comprehensive explanation of fluid behavior. Appreciable improvement was brought about by the introduction of a third parameter, a parameter based on molecular structure. The most commonly used parameter that represents this quantity is known as the acentric factor $(\omega)$. The acentric factor can be determined for any fluid from the critical temperature, critical pressure, and a single vapor-pressure measurement made at $T_{\mathrm{r}}=0.7$ using the following equation (Smith et al., 2002):

$$
\omega \equiv-1.0-\log \left(P_{r}^{s a t}\right)_{T_{r}=0.7}
$$

However, values for the acentric factor for particular substances are listed in numerous technical sources. With the introduction of the acentric factor, the threeparameter theorem of corresponding states reads as follows: All fluids having the same value of $\omega$, when compared at the same reduced temperature and pressure, have about the 
same value of $Z$ (compressibility factor), and all deviate from ideal-gas behavior to about the same degree. This theorem validates the Peng-Robinson and Reidlich-Kwong-Soave equations of state (Smith et al., 2002).

\section{B. The van der Waals Equation of State and Some Simple Modifications}

The pressure-explicit form of the original van der Waals equation of state, in terms of temperature and gas molar volume, is (TABLE I):

$$
P=R T /(v-b)-a / v^{2}
$$

Parameters $a$ and $b$ are constants whose values are determined from the measured critical pressure value $\mathrm{P}_{\mathrm{c}}$ and the critical temperature value $\mathrm{T}_{\mathrm{c}}$. The parameter $a$ is a molar enhancement factor that accounts for attractive interactions between the molecules while the parameter $b$ accounts for the atomic volume of the gaseous molecules. While these parameters improve upon thermodynamic computation from the ideal gas law, they are only really valid for data at the critical conditions. It turns out that the parameters $a$ and $b$ are not constant. Both are dependent upon the temperature and the gas concentration, or gas molar density $(\rho=1 / v)($ McNeill et al., 2004). TABLE II below summarizes the changes made to the $a$ and $b$-parameters from the original van der Waals equation of state to generate the modified van der Waals equation. 
TABLE II

\section{MODIFICATIONS TO VAN DER WAALS PARAMETERS}

\begin{tabular}{|c|c|}
\hline Original van der Waals & Modified van der Waals \\
\hline $\mathrm{a}=(27 / 64)^{*}\left(\mathrm{R}^{*} \mathrm{~T}_{\mathrm{c}}{ }^{2} / \mathrm{P}_{\mathrm{c}}\right)$ & $\mathrm{a}_{\mathrm{HS}}=\mathrm{a}_{1}-\mathrm{a}_{\mathrm{c}}{ }^{*}\left(T / \mathrm{T}_{\mathrm{c}}\right)$ \\
\hline $\mathrm{b}=(1 / 8)^{*}\left(\mathrm{R}^{*} \mathrm{~T}_{\mathrm{c}} / \mathrm{P}_{\mathrm{c}}\right)$ & $b_{H S}=\left(b_{b} / 4\right) *\left[1+\sqrt{1+2 b_{0}{ }^{2}\left(\rho-2 / b_{0}\right)^{2}}\right]$ \\
\hline & $\mathrm{b}_{\mathrm{b}}=0.18^{*}\left(\mathrm{R}^{\star} \mathrm{T}_{\mathrm{c}} / \mathrm{P}_{\mathrm{c}}\right)$ \\
\hline
\end{tabular}

Parameter $a$ accounts for attractive forces at a certain distance between real gas

atoms. Furthermore, the $a$-parameter will be essentially temperature dependent (because the dependence upon gas molar density is negligible compared to the temperature dependence) (McNeill et al., 2004). Conversely, the $b$-parameter involves the repulsive forces taking place between gaseous atoms during the moment of collision. As a result, this parameter will be mostly dependent upon the gas concentration $(1 / v)$, which is given is units of $\mathrm{mol} / \mathrm{cm}^{3}$ (McNeill and Collins, 2004). In order to determine the dependence of the $b$-parameter upon the molar gas density, $\rho=1 / \nu$, the hard-sphere model for gases was implemented. The hard-sphere model is based upon the assumption that atoms and molecules behave like bouncing hard spheres as illustrated in FIGURE 2. 


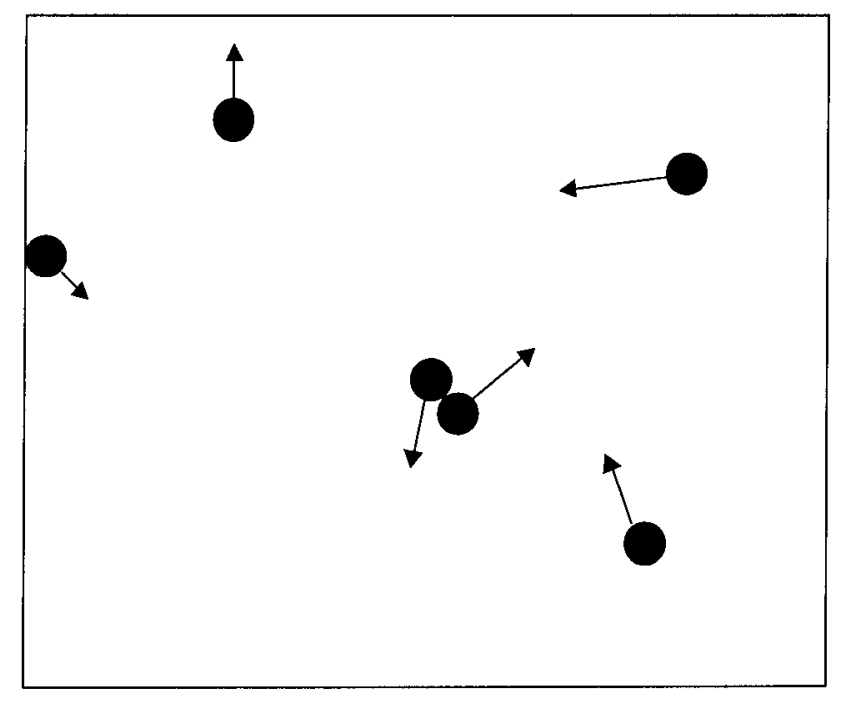

FIGURE 2 - Hard Sphere Model for Modified VDW

The spheres are incompressible and there are no attractive, frictional, and gravitational forces between the spheres. When applying the hard-sphere model to the van der Waals equation of state, only repulsive forces exist at the moment of collision. After a collision, momentum and energy are conserved. The equation of state for the hard-sphere model is similar to the van der Waals expression except that there is no negative term relative to attractive interactions (McNeill and Collins, 2004):

$$
P=R T /\left(v-b_{H S}\right)
$$

Molecular dynamics computer simulations were run using noble gases to determine the $b$-parameter dependence upon the molar gas density. Noble gases are a special case because they are inert; noble gases are nonpolar atoms that have no atomic forces. As stated previously, the hard-sphere model assumes that gas atoms are bouncing like 
billiard balls in space with no attractive interactions at a distance. In order to compute the atomic volume $\left(v_{a}\right)$ of the gas, the diameter of the hard-sphere atom is set equal to the Lennard-Jones potential $\sigma$-parameter obtained from the viscosity data (Prausnitz et al., 1999):

$$
v_{a}=4 \pi(\sigma / 2)^{3} / 3
$$

Gas atoms are initially arranged within the container by the simple cubic unit cell structure. A Gaussian random number generator is utilized to achieve the Maxwellian velocity distributions. By performing simulations using the hard-sphere model for large numbers of gas atoms, the numerical value of the total atomic volume, $b_{H S}$, can be evaluated at different values of molar volume from the observed gas pressure P. During the computer simulation, the gas pressure $\mathrm{P}$ is determined from the collision rate of hardsphere gas atoms with the six cubic walls of the "control volume". Once the pressure is measured, the effective atomic molar volume is calculated by the following (McNeill and Collins, 2004)

$$
b_{H S}=N_{A} v_{e f f}
$$

where $\mathrm{N}_{\mathrm{A}}$ is Avogadro's number and $v_{\mathrm{eff}}$ is the effective atomic volume based on the above experimentation at pressure $\mathrm{P}$.

By analyzing the changing value of parameter $b_{H S}$ with respect to changing gas molar volume, it is possible to derive an expression that approximately models the 
dependence of van der Waals parameter $b$ with the molar gas volume $v$. To obtain a fit for the effective molar volumes $b_{H S}$ observed in these simulations as the molar gas density was increased, the following equation of a hyperbola was found to be the most appropriate when utilizing the simple cubic unit cell arrangement in the initial positioning of gas atoms (McNeill and Collins, 2004):

$$
b_{H S}=\left(b_{0} / 4\right) *\left[1+\sqrt{1+2 b_{0}^{2}\left(\rho-2 / b_{0}\right)^{2}}\right]
$$

The parameter $b_{0}$ is set equal to four times the actual atomic molar volume $b_{A V}$, or the total sum of atomic volumes in one mole of a monatomic gas (McNeill and Collins, 2004):

$$
b_{0}=4 b_{A V}=4 N_{A} v_{a}
$$

The maximum value for the expression of $b_{H S}$ is $b_{0}$, which occurs at zero pressures when the gas molar density $(\rho)$ is zero. The minimum value of $b_{H S}$ is $b_{0} / 2$ and occurs at infinite pressures when the minimum gas molar volume is also $b_{0} / 2$ (McNeill and Collins, 2004).

Similarly, for all other pure substances, if one substitutes the preceding expression for $b_{H S}$ into the original van der Waals equation of state, the parameters to be determined from the measured critical pressure and the measured critical temperature are $a$ and $b_{0}$. Using differential calculus, it can be shown that for the hard sphere model the values of parameters $b_{0}$ and $a_{0}$ upon the measured critical data are (McNeill and Collins, 2004): 


$$
\begin{gathered}
P=R T /\left(v-b_{H S}\right)-a_{0} / v^{2} \\
b_{0}=\left(R T_{c} / P_{c}\right) / 5.557586863 \\
a_{0}=\left(R^{2} T_{c}^{2} / P_{c}\right) / 2.04504859
\end{gathered}
$$

Six compounds were studied during the course of the analysis: argon, ethane, propane, $n$ butane, $n$-pentane, and water. The $b$ values for these substances calculated by equation 14, equation 2 , and from simulations at $298 \mathrm{~K}$ and gas molar density of one mole per liter (which simulates zero pressure conditions) are shown in TABLE III.

\section{TABLE III}

\section{COMPARISON OF VAN DER WAALS $b$-PARAMETERS}

\begin{tabular}{|c|c|c|c|}
\hline Compound & $\mathbf{b}_{\circ}\left(\mathbf{c m}^{3} / \mathbf{~ m o l}\right)$ & $\mathbf{b}\left(\mathbf{c m}^{3} / \mathbf{m o l}\right)$ & $\mathbf{b}_{\mathrm{HS}}\left(\mathbf{c m}^{3} / \mathbf{m o l}\right)$ \\
\hline Argon & 46.06 & 32.00 & 51.00 \\
\hline Ethane & 93.74 & 65.12 & \\
\hline Propane & 130.23 & 90.47 & \\
\hline$n$-Butane & 167.53 & 116.38 & \\
\hline$n$-Pentane & 208.50 & 144.85 & \\
\hline Water & 43.89 & 30.49 & \\
\hline
\end{tabular}

Once this variation in the $b$-parameter was verified, the temperature dependence of the parameter $a$ in the modified van der Waals gas equation was determined. First, values of the parameter $a$ were obtained at different temperatures using the measured equilibrium liquid vapor pressure data listed in Perry's Chemical Engineers' Handbook, $7^{\text {th }}$ Edition (1997). At the critical point, parameters $a_{0}$ and $b_{0}$ were determined using the condition of criticality. While $b_{0}$ is kept constant, the $a$-parameter was re-evaluated using 
the integral expression below for each different measured liquid vapor pressure value $\mathrm{P}_{\text {equilibrium }}$ for specified temperatures $\mathrm{T}$ such that $\mathrm{T}<\mathrm{T}_{\mathrm{c}}(\mathrm{McNeill}$ and Collins, 2004):

$$
\int_{v_{l}}^{v_{s}} P(T, v) d v=\int_{v_{l}}^{v_{g}} R T /\left(v-b_{H S}\right) d v-\int_{v_{l}}^{v_{k}} a_{H S} / v^{2} d v=P_{\text {equil }}\left(v_{g}-v_{l}\right)
$$

The values for the universal gas constant (R), temperature (T), and equilibrium gas pressure $\left(\mathrm{P}_{\text {equil }}\right)$ are all specified. The values for the vapor and liquid molar volumes $\left(v_{\mathrm{g}}\right.$ and $v_{1}$ ) are obtained from Perry's Chemical Engineers' Handbook, $7^{\text {th }}$ Edition (1997). The value for $b_{H S}$ is calculated from $b_{0}$ and the specified $v_{\mathrm{g}}$. Using differential calculus, the value of $a_{H S}$ can be determined. By observing plots of the $a$-parameter versus temperature, the dependence of the $a$-parameter upon temperature is essentially linear with a negative slope (McNeill and Collins, 2004):

$$
a_{H S}=a_{1}-a_{2}\left(T / T_{c}\right)
$$

Note that at the critical temperature, $\mathrm{T}=\mathrm{T}_{\mathrm{c}}$ and $a_{H S}=a_{l}-a_{2}=a_{0}$. The numerical values for parameters $a_{1}$ and $a_{2}$ can be computed utilizing a linear least squares fit. The experimentally determined values of $a_{1}$ and $a_{2}$ for the six previously mentioned compounds are as follows in TABLE IV (McNeill and Collins, 2004): 
TABLE IV

MODIFIED VAN DER WAALS $a$-PARAMETERS

\begin{tabular}{|c|c|c|}
\hline Compound & $\mathbf{a}_{\mathbf{1}}\left(\mathbf{b a r}^{*} \mathbf{c m}^{\mathbf{6}} / \mathbf{m o l}^{\mathbf{2}}\right)$ & $\mathbf{a}_{\mathbf{2}}\left(\mathbf{b a r}^{*} \mathbf{c m}^{\mathbf{6}} / \mathbf{m o l}^{2}\right)$ \\
\hline Argon & $1.35 \mathrm{E}+06$ & 0 \\
\hline Ethane & $7.69 \mathrm{E}+06$ & $1.18 \mathrm{E}+06$ \\
\hline Propane & $1.39 \mathrm{E}+07$ & $2.97 \mathrm{E}+06$ \\
\hline n-Butane & $2.22 \mathrm{E}+07$ & $5.35 \mathrm{E}+06$ \\
\hline n-Pentane & $3.09 \mathrm{E}+07$ & $8.75 \mathrm{E}+06$ \\
\hline Water & $6.68 \mathrm{E}+06$ & $6.07 \mathrm{E}+06$ \\
\hline
\end{tabular}

\section{Thermodynamic Properties from Equations of State}

As stated before, equations of state are important because they provide a starting point for the evaluation of thermodynamic properties of substances. These thermodynamic properties include, but are not limited to, internal energy, enthalpy, and entropy. Furthermore, the cornerstone thermodynamic property from which all other are derived is known as the Gibbs Energy (G). Unfortunately, there is no known experimental method for determining numerical values of Gibbs energy for a particular substance. However, the concept that the Gibbs energy can be used to generate all other thermodynamic properties leads to the definition of a closely related property for which numerical values are readily obtained. This property is known as a residual property. The residual Gibbs energy, $G^{R}$, is defined by (Smith et al., 1996):

$$
G^{R} \equiv G-G^{i g}
$$


$\mathrm{G}$ and $\mathrm{G}^{\mathrm{ig}}$ are the actual and the ideal-gas values of the Gibbs energy at the same temperature and pressure. In fact, the general definition for a residual property is (Smith et al., 1996):

$$
M^{R} \equiv M-M^{i g}
$$

$M$ denotes the molar value of any extensive thermodynamic property $-v, U, S, G$, etc. The residual property quantifies the difference in thermodynamic properties of a real gas and that gas at ideal conditions (for the same temperature and pressure). In other words, the residual property accounts for the non-ideality of a gas. Using fundamental property relationships and Maxwell's relations, the residual enthalpy of a gas is (note that the derivative of the compressibility is taken at constant total molar volume) (Smith et al., 1996):

$$
H^{R} / R T=(Z-1)+T \int_{\infty}^{v}(\partial Z / \partial T)_{v} * d v / v
$$

Once the residual enthalpy has been calculated, the total enthalpy is determined by (Smith et al., 1996):

$$
H=H_{0}{ }^{i g}+\int_{T_{0}}^{T} C_{p}{ }^{i g}(d T / T)+H^{R}
$$


$\mathrm{H}_{0}{ }^{\mathrm{ig}}$ is a standard-state enthalpy for an ideal gas taken at a reference condition of $\mathrm{T}_{0}$ and $\mathrm{P}_{0} . \mathrm{C}_{\mathrm{p}}{ }^{\mathrm{ig}}$ is determined from the following equation in Reklaitis (1983):

$$
c_{p}=a+b T+c T^{2}+d T^{3}+e T^{4}
$$

Constants a through e come from fitting polynomial regressions of $\mathrm{C}_{\mathrm{p}}^{\mathrm{ig}} \mathrm{vs}$. $\mathrm{T}$ and are listed in Reklaitis (1983).

The application of residual properties is most advantageous for gases because residual properties are quite small in comparison to the ideal-gas thermodynamic properties. It is not as effective when applied to liquids due to the large enthalpy and entropy changes due to vaporization. The fundamental theory behind residual properties can be applied to any equation of state - because they are continuous functions that can be integrated and differentiated. For example, for the Reidlich-Kwong equation of state (TABLE I):

$$
P=R T /(v-b)-a /\left[T^{1 / 2} * v(v+b)\right]
$$

Rearrangement provides a form explicit in terms of compressibility (Smith et al., 1996):

$$
\begin{gathered}
Z=1 /(1-h)-\left(1 / b R T^{1.5}\right) *[h /(1+h)] \\
\text { where } h \equiv b P / Z R T \equiv b / v
\end{gathered}
$$


By algebraic manipulation and the use of differential calculus, it can be shown that the residual enthalpy for a gas at a particular pressure and temperature is given by (Smith et al., 1996):

$$
H^{R}=(Z-1)-\left(3 a / 2 b R T^{1.5}\right) * \ln (1+h)
$$

TABLE $V$ lists the equations for the residual enthalpy for different equations of state; details of these derivations are listed in Appendix III.

\section{TABLE V}

RESIDUAL ENTHALPIES FOR EQUATIONS OF STATE

\begin{tabular}{|c|c|}
\hline Original van der Waals & $H^{R} / R T=[b /(v-b)]-(2 a / V R T)$ \\
\hline & $a=(27 / 64) *\left(R^{2 *} T_{c}^{2} / P_{c}\right)$ \\
\hline & $\mathrm{b}=(1 / 8)^{*}\left(\mathrm{R}^{*} \mathrm{~T}_{\mathrm{c}} / \mathrm{P}_{\mathrm{c}}\right)$ \\
\hline \multirow[t]{4}{*}{ Reidlich-Kwong } & $H^{R} / R T=[h /(1-h)]-\left(a / b R T^{1.5}\right)^{*}\left\{[h /(1+h)]+1.5^{*} \ln (1+h)\right\}$ \\
\hline & $\mathrm{h}=\mathrm{bP} / \mathrm{ZRT}=\mathrm{b} / \mathrm{v}$ \\
\hline & $a=0.42748 *\left(R^{2 *} T_{c}^{2.5} / P_{c}\right)$ \\
\hline & $\mathrm{b}=0.08664 *\left(\mathrm{R}^{\star} \mathrm{T}_{\mathrm{c}} / \mathrm{P}_{\mathrm{c}}\right)$ \\
\hline Modified van der Waals & $H^{\mathrm{R}} / \mathrm{RT}=\left[\mathrm{b}_{\mathrm{HS}} /\left(\mathrm{v}-\mathrm{b}_{\mathrm{HS}}\right)\right]-\left(2 \mathrm{a}_{1} / \mathrm{VRT}\right)+\left(\mathrm{a}_{2} / \mathrm{VRT}_{\mathrm{c}}\right)$ \\
\hline
\end{tabular}




\section{METHODS}

For each of the six compounds listed in Chapter II (argon, water, ethane, propane, $n$-butane, and $n$-pentane), physical property charts were created relating molar volume $(v)$ and molar enthalpy $(\mathrm{H})$ as a function of pressure and temperature. Examples of these types of charts are illustrated in FIGURES 3 AND 4. Values used to create these charts were calculated using Microsoft Excel and Microsoft VBA. For each compound, the Reidlich-Kwong-Soave, Reidlich-Kwong, original van der Waals, and modified van der Waals equations of state were utilized to create pressure-molar volume (Pv) charts; with regards to the pressure-enthalpy $(\mathrm{PH})$ charts, only the latter three equations of state were used. Given below is a sample spreadsheet that illustrates how the gas and liquid molar volumes were calculated for ethane using the original VDW equation of state:

\section{TABLE VI}

EQUILIBRIUM VAPOR AND LIQUID MOLAR VOLUMES USING THE VAN DER

\section{WAALS EQUATION OF STATE}

\begin{tabular}{|c|c|c|c|c|c|c|c|c|c|c|c|c|c|c|c|c|c|c|}
\hline$T(4)$ & $P_{n=\left(K P_{1}\right)}$ & $P_{-m}(\mathrm{ber})$ & $R\left(\mathrm{sin}^{3} \mathrm{bar} / \mathrm{mol}+\mathrm{k}\right)$ & $T_{c}(K)$ & $P_{f}\left(b=b_{1}\right)$ & $T_{t}$ & $\mathbf{P}_{\mathrm{r}}$ & $\alpha\left(T_{i}\right)$ & $\psi$ & - $\left.\left(\mathrm{burcm}^{8} / \mathrm{mol}\right)^{2}\right)$ & $\Omega 2$ & $0\left(a m^{3} / m a d\right)$ & $v_{\text {rad }}(\mathrm{em})$ & $P$ (bar) & $V_{\text {nqpaqu }}\left(\mathrm{cm}^{2}\right)$ & $V_{\operatorname{lig} 00}\left(\alpha m^{3}\right)$ & $P($ man $)$ & 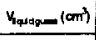 \\
\hline 1845 & 1008 & 1000 & 83.14 & 306.3 & 4872 & 0.804 & 0.021 & 1.000 & $0.4218 \pi$ & $5,578,520$ & 0.125 & 5.12 & 14007.7 & 1.008 & 14801.1 & 04.9 & 1.008 & 84.9 \\
\hline 190 & 1346 & 1346 & 8314 & 305.3 & 4672 & 0.522 & 0008 & 1000 & $0.4218 \pi$ & $5,578,920$ & 0.125 & 86.12 & 11437.5 & 1.346 & $1+2848$ & 8.1 & 1.346 & 86.1 \\
\hline 200 & 217.9 & 2.179 & 83.14 & 306.3 & 4872 & 0.65 & 0.045 & 1.000 & $0.4218 \pi$ & $5,578,920$ & 0.125 & 85.12 & 7340,3 & 2179 & 7343.4 & $\infty .3$ & 2.179 & क.3. \\
\hline 210 & 336.0 & 3.60 & 83.14 & 305.3 & 4872 & 0.889 & 0069 & 1.000 & $0.4218 \pi$ & $5,578.900$ & 0.125 & Ð. 12 & $\$ 28.6$ & 3350 & 48253 & 90.0 & 3.300 & 90.8 \\
\hline 230 & 496.7 & 4.967 & 83.14 & 306.3 & 4672 & 0.721 & 0.102 & 1.000 & $0.4218 \pi$ & $5,678,900$ & 0.125 & 65.12 & 3425.4 & 4968 & 3423.3 & $\$ 0.7$ & 4.987 & 93.7 \\
\hline 230 & 708.3 & 7083 & 8914 & 306.3 & 4872 & 0.733 & 0.146 & 1.000 & $0.4218 \pi$ & $5,576,900$ & 0.125 & 65.12 & 24seo & 7008 & 2450.7 & 9.0 & 7.083 & 97.0 \\
\hline 240 & 978.7 & 9787 & 83.14 & 306.3 & 4672 & 0.786 & 0201 & 1.000 & $0.4218 \pi$ & $5,578,920$ & 0.125 & ๔.12 & 1796.2 & $97 \circledast$ & 1797.3 & 1009 & 9.87 & 100.9 \\
\hline 250 & 1315.9 & 13.199 & 83.14 & 305.3 & 4872 & 0.819 & 0.270 & 1.000 & $0.4218 \pi$ & $5,578,9 D$ & 0.125 & क5. 12 & 13446 & 13.159 & 1344.6 & 105.6 & 13.160 & 105.6 \\
\hline 260 & 1727.2 & 17.272 & 83.14 & 305.3 & 4872 & 0.062 & 0.366 & 1000 & 0.421875 & $5,578,920$ & 0.25 & 05.12 & mon.2 & 17.73 & 1020.2 & 111.3 & 17.272 & 111.5 \\
\hline 270 & 2219.5 & 22190 & 83.14 & 306.3 & 4872 & 0.084 & 0.456 & 1.000 & 0.421875 & $5,578,92 D$ & 0.125 & $\infty 6.12$ & 701.8 & 22.196 & 781.8 & 119.5 & 22195 & 119.5 \\
\hline 200 & 2786.9 & 27.508 & 89.14 & 306.3 & 4872 & 0.917 & $05 / 4$ & 1000 & 0.421875 & $5,578,920$ & 0.125 & $\infty 12$ & $\$ 01.2$ & 27.090 & 601.2 & 131.8 & 27.999 & 131.8 \\
\hline 290 & 3407 & 34707 & 83.14 & 306.3 & 4672 & 0.550 & 0.712 & 1.000 & 0.421875 & $5,57,920$ & 0.125 & 66.12 & 4897 & 34.707 & 459.7 & 1535 & 36.044 & 15300 \\
\hline 298 & 38427 & 38.427 & 83.14 & 3053 & 4872 & 0.956 & 0.789 & 1.000 & $0.4218 \pi$ & $5,578,920$ & 0.125 & 6512 & 300.8 & 30.6 & 398.8 & 1589.9 & 40.582 & 1590 \\
\hline 300 & 420.5 & 42300 & 83.14 & 306.3 & 4872 & 0.563 & 080 & 1.000 & $0.218 \pi$ & $5,579,90$ & 0.125 & Eक 12 & 341.8 & 42.34 & 341.8 & 1692 & 4.779 & 168.0 \\
\hline 3063 & 4687.6 & $46.8 \%$ & 83.14 & 305.3 & 4672 & 1.000 & 0.962 & 1.000 & $0.4218 \pi$ & $5,579,920$ & 0.125 & E6 12 & 2022 & 46.876 & 2822 & 2022 & 46.876 & 2222 \\
\hline
\end{tabular}




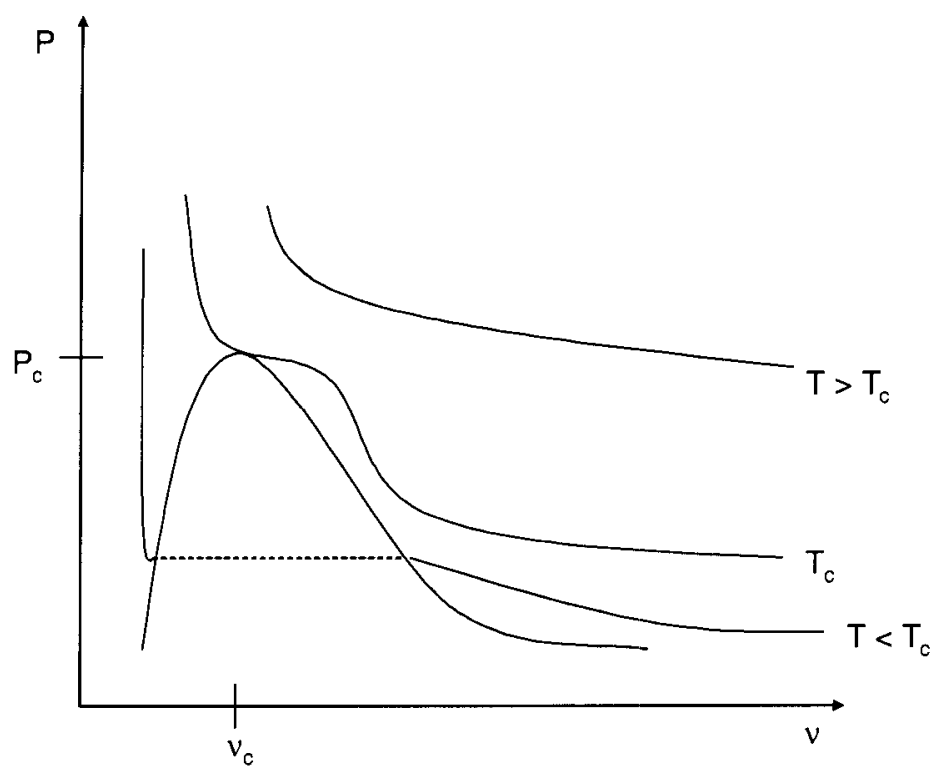

FIGURE 3 - Theoretical Pressure-Volume Diagram

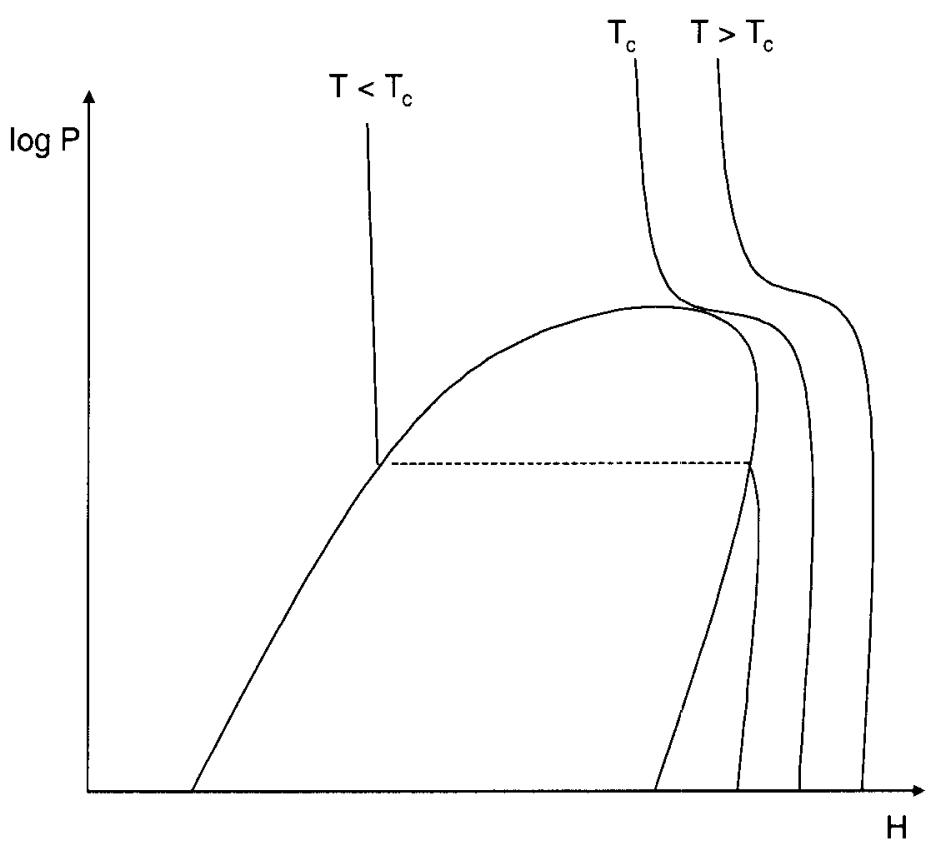

FIGURE 4 - Theoretical Log Pressure-Enthalpy Diagram 
Equilibrium conditions were analyzed first, over a range of temperatures beginning with the normal boiling point and ending at the critical point; temperatures between the endpoints were chosen at random intervals. The equilibrium pressure, otherwise known as the saturation pressure $\left(\mathrm{P}^{\mathrm{sat}}\right)$, was calculated at each temperature using the Antoine Equation (Reklaitis, 1983):

$$
\ln P^{s a t}=A-B /(T+C)
$$

The temperature $(\mathrm{T})$ must be in degrees Kelvin, while the pressure $(\mathrm{P})$ is given in kilopascals $(\mathrm{kPa})$. The constants $\mathrm{A}, \mathrm{B}$, and $\mathrm{C}$ are coefficients curved-fitted from experimental pressure and temperature data and given in Reklaitis (1983). After solving the Antoine Equation for pressure (P), appropriate constants pertinent to each equation of state $\left(\mathrm{R}, \mathrm{T}_{\mathrm{c}}, \mathrm{P}_{\mathrm{c}}, \omega, \mathrm{a}_{1}, \mathrm{a}_{2}\right.$, etc.) were employed to calculate values for $a$ (units of bar* $\mathrm{cm}^{6} /$ $\mathrm{mol}^{2}$ ) and $b$ (units of $\mathrm{cm}^{3} / \mathrm{mol}$ ); for the modified VDW equation of state, $a_{0}$ and $b_{0}$ were determined. The subsequent column of cells were independent values that could be entered arbitrarily; these cells were labeled $V_{\text {vaporguess. }}$ These cells serve as the initial values for the iterations to be described below.

The next two columns of cells calculate the pressure and molar volume of the gas based upon the constants previously calculated in addition to the cell that supplies the initial guess. Listed in the TABLE VII are the pressure-explicit and molar volumeexplicit forms of the equation used for the vapor phase (Smith et al., 2002): 
TABLE VIII

EQUATIONS OF STATE IN VAPOR MOLAR VOLUME EXPLICIT FORM

\begin{tabular}{|c|c|}
\hline Original van der Waals & $\mathrm{P}=(\mathrm{RT} / \mathrm{v}-\mathrm{b})-\left(\mathrm{a} / \mathrm{v}^{2}\right)$ \\
\cline { 2 - 2 } Modified van der Waals & $\mathrm{v}=(\mathrm{RT} / \mathrm{P})+\mathrm{b}-\left\{(\mathrm{a} / \mathrm{P})^{\star}\left[(\mathrm{v}-\mathrm{b}) / \mathrm{v}^{2}\right]\right\}$ \\
\hline Reidlich-Kwong & $\mathrm{P}=(\mathrm{RT} / \mathrm{v}-\mathrm{b})-\left(\mathrm{a} / \mathrm{v}^{*}(\mathrm{v}+\mathrm{b})\right)$ \\
\cline { 2 - 2 } Reidlich-Kwong-Soave & $\mathrm{v}=(\mathrm{RT} / \mathrm{P})+\mathrm{b}-\left\{(\mathrm{a} / \mathrm{P})^{*}\left[(\mathrm{v}-\mathrm{b}) / \mathrm{v}^{*}(\mathrm{v}+\mathrm{b})\right]\right\}$ \\
\hline
\end{tabular}

The correct pressure and molar volume for each given temperature-pressure equilibrium data point was calculated using the Goal Seek tool in Microsoft Excel. The pressure value calculated from the applicable equation of state was set equal to the pressure calculated from the Antoine Equation by changing the cell that contained the initial guess. Excel continued to perform successive iterations until the two pressures were equal. As a check of this loop, the calculated molar volume should be equal to the cell that once contained the initial guess for the molar volume (which also now contains the correct final molar volume). Instead of executing the Goal Seek tool for each set of data points, a simple macro was written in Microsoft VBA to execute the Goal Seek tool for all sets of data points simultaneously. A small sample of the macro is shown below:

Sub RKS_Vapor_UseGoalSeek()

With Worksheets("RKS")

.Range("P6").GoalSeek

goal:=.Range("C6").Value, _

ChangingCell:=Range("Q6")

.Range("P7").GoalSeek

goal:=.Range("C7").Value, _ 
ChangingCell:=Range("Q7")

.Range("P8").GoalSeek_

goal:=.Range("C8").Value,

ChangingCell:=Range("Q8")

.Range("P9").GoalSeek_

goal:=.Range("C9").Value, _

ChangingCell:=Range("Q9")

.Range("P10").GoalSeek _

goal:=.Range("C10").Value, _

ChangingCell:=Range("Q10")

End With

End Sub

Once the gas molar volumes were determined, a similar procedure was incorporated in order to calculate the corresponding liquid molar volumes for the sets of equilibrium data points. The major difference is that the molar volume-explicit equations for the equations of state are given as the following (Smith et al., 2002):

\section{TABLE VIII}

EQUATIONS OF STATE IN LIQUID MOLAR VOLUME EXPLICIT FORM

\begin{tabular}{|c|c|}
\hline Original van der Waals & $P=(R T / v-b)-\left(a / v^{2}\right)$ \\
\cline { 2 - 2 } Modified van der Waals & $v=b+\left\{v^{2}[(R T+b P-v P) / a]\right\}$ \\
\hline Reidlich-Kwong & $P=(R T / v-b)-\left(a / v^{*}(v+b)\right)$ \\
\cline { 2 - 2 } Reidlich-Kwong-Soave & $v=b+\left\{\left[v^{*}(v+b)\right]^{*}[(R T+b P-v P) / a]\right\}$ \\
\hline
\end{tabular}


With the aforementioned exception, the process for calculating the correct pressures and liquid molar volumes via the Goal Seek tool are exactly the same. In addition to vaporliquid equilibrium conditions, pressure-molar volume behavior was examined under constant temperature conditions. Arbitrary temperatures were chosen above the critical temperature as well as below the critical temperature in the equilibrium region. For each temperature, the pressure was varied over a range of 1 bar (essentially atmospheric pressure) to approximately twice the critical pressure of the specific compound. Gas and liquid molar volumes, where applicable, were determined for these isotherms using the Goal Seek method previously mentioned. Consequently, the equilibrium gas and liquid molar volumes were plotted on the $\mathrm{x}$-axis versus pressure, located on the $\mathrm{y}$-axis; also on these graphs were relationships of molar volume versus pressure at each of the isotherms.

Once the $\mathrm{P} v$ relationships were prepared, attention was turned to developing pressure-enthalpy relationships, an example of which is shown in TABLE IX. In order to maintain cohesion, the pressures and temperatures used in developing the P $v$ diagrams were used again for the PH diagrams. Enthalpy is categorized as a state property, which is a property that does not depend on the past history of a substance nor by the means by which it reaches a given state. State properties are functions of present conditions, however reached. A state property always has a value; it can be expressed mathematically as a function of other thermodynamic properties (Smith, et al., 2002). Hence, an arbitrary zero enthalpy has to be stated. This zero enthalpy is otherwise known as a standard-state enthalpy. Standard-state enthalpy was chosen as a liquid at the compound's normal boiling point, or nbp (temperature at which the saturation pressure is equal to atmospheric pressure). The first step in determining the total enthalpy of each 
TABLE IX

EQUILIBRIUM VAPOR AND LIQUID MOLAR ENTHALPIES FOR ETHANE USING THE ORIGINAL VAN DER WAALS

EQUATION OF STATE

\begin{tabular}{|c|c|c|c|c|c|c|c|c|c|c|c|c|c|c|c|}
\hline$T(K)$ & $P_{\text {sot }}($ bar $)$ & $\mathbf{T}_{\mathbf{r}}$ & $\mathbf{P}_{\mathrm{r}}$ & $\mathrm{T}_{\text {rot }}(\mathrm{K})$ & $\mathrm{C}_{\mathrm{p}, \text { (avg) }}-\mathrm{J} / \mathrm{mol}{ }^{\prime} \mathrm{K}$ & $\Delta \mathrm{H}_{\operatorname{mop}}(\mathrm{J} / \mathrm{mol})$ & $\Delta H_{\mathrm{ppg}}(\mathrm{J} / \mathrm{mol})$ & $a\left(\operatorname{bar}^{*} c \pi^{6} / \mathrm{mol}^{2}\right)$ & $\mathrm{b}\left(\mathrm{cm}^{3} / \mathrm{mol}\right)$ & $V_{\text {vepor }}\left(\mathrm{cm}^{3}\right)$ & $\mathrm{H}_{1}{ }_{1}(\mathrm{~J} / \mathrm{mol})$ & $\mathrm{H}_{2}(\mathrm{~J} / \mathrm{mol})$ & $\Delta \mathrm{H}_{\text {map }}(\mathrm{J} / \mathrm{mol})$ & $H_{\text {sectrap }}(\mathrm{J} / \mathrm{mol})$ & $\mathrm{H}_{\text {satd iq }}(\mathrm{J} / \mathrm{mol})$ \\
\hline 184.5 & 1.008 & 0.604 & 0.021 & 184.5 & 41.375 & 14715.6 & 0.0 & $5,578,920$ & 65.12 & 14907.7 & $-\infty .1$ & -68.1 & 14715.6 & 14715.6 & 0.0 \\
\hline 190 & 1.346 & 0.622 & 0.028 & 184.5 & 41.612 & 14715.6 & 228.9 & $5,578,920$ & 65.12 & 11437.5 & $-\infty .1$ & -88.5 & 14457.3 & 14924.1 & 466.8 \\
\hline 200 & 2.179 & 0.655 & 0.045 & 184.5 & 42055 & 14715.6 & 651.8 & $5,578,920$ & 65.12 & 7349.3 & -68.1 & -137.0 & 13967.4 & 15298.6 & 1331.2 \\
\hline 210 & 3.360 & 0.688 & 0.069 & 184.5 & 42514 & 14715.6 & 1084.1 & $5,578,920$ & 65.12 & 4928.6 & -68.1 & -203.0 & 13447.7 & 15664.8 & 2217.1 \\
\hline 200 & 4.967 & 0.721 & 0.102 & 184.5 & 42989 & 14715.6 & 1526.1 & $5,578,920$ & 65.12 & 3425.4 & -68.1 & -290.3 & 12893.0 & 16019.5 & 3126.6 \\
\hline 230 & 7.083 & 0.753 & 0.145 & 184.5 & 43.478 & 14715.6 & 1978.3 & $5,578,920$ & 65.12 & 24520 & $-\infty .1$ & -402.9 & 12296.3 & 16359.1 & 40628 \\
\hline 240 & 9.787 & 0.786 & 0.201 & 184.5 & 43.981 & 14715.6 & 2441.0 & $5,578,920$ & 65.12 & 1798.2 & -68.1 & -545.5 & 11648.2 & 16679.1 & 5030.9 \\
\hline 250 & 13.159 & 0.819 & 0.270 & 184.5 & 44.497 & 14715.6 & 2914.6 & $5,5 / 8,920$ & 65.12 & 1344.6 & -68.1 & -724.0 & 10935.2 & 16974.2 & 6039.0 \\
\hline 260 & 17.272 & 0.852 & 0.355 & 184.5 & 45.025 & 14715.6 & 3399.4 & $5,578,920$ & 65.12 & 1020.2 & $-\infty .1$ & -946.3 & 10137.0 & 17236.8 & 7099.8 \\
\hline 270 & 22.195 & 0.884 & 0.456 & 184.5 & 45.564 & 14715.6 & 3895.7 & $5,578,920$ & 65.12 & 781.8 & -68.1 & -1223.3 & 9220.3 & 17456.2 & 8235.8 \\
\hline 280 & 27.989 & 0.917 & 0.574 & 184.5 & 46.114 & 14715.6 & 4403.8 & $5,578,920$ & 65.12 & 601.2 & -68.1 & -1573.0 & 8124.2 & 17614.6 & 9490.4 \\
\hline 290 & 34.707 & 0.950 & 0.712 & 184.5 & 46.673 & 14715.6 & 4924.0 & $5,578,920$ & 65.12 & 459.7 & -68.1 & -2029.4 & 6710.8 & 17678.3 & 10967.4 \\
\hline 295 & 38.427 & 0.966 & 0.789 & 184.5 & 46.956 & 14715.6 & 5188.6 & $5,578,920$ & 65.12 & 398.8 & $-\infty .1$ & -2319.3 & 5773.9 & 17653.0 & 11879.1 \\
\hline
\end{tabular}


compound at a specific temperature and pressure is to determine the enthalpy of vaporization at its nbp. The enthalpy of vaporization for a substance at its normal boiling point is given in Reklaitis (1983). Once the liquid is vaporized, the compound becomes a saturated vapor in the real-gas state. In order to convert the vapor to an ideal-gas state, the residual enthalpy (which is actually a negative quantity) must be subtracted from the enthalpy of vaporization at the nbp. Recall that residual enthalpy is calculated for the original VDW equation of state by the following (Smith et al., 2002):

$$
H^{R}=R T\{[b /(v-b)]-(2 a / v R T)\}
$$

Now the vapor is in an ideal gas-state. Enthalpy necessary to convert the ideal gas from its reference temperature to its actual temperature comes from the heat capacity $\left(c_{p}\right)$ and is defined by (Smith et al., 2002)

$$
\Delta H=c_{p, a v g}\left(T_{\text {final }}-T_{\text {intial }}\right)
$$

where $c_{p}$ is calculated by the following, obtained from Reklaitis (1983):

$$
c_{p}^{i g}=a+b T+c T^{2}+d T^{3}+e T^{4}
$$

Constants a through e are fitted polynomial regressions from experimentally determined quantities of temperature and heat capacity listed in Reklaitis (1983). The heat capacity 
was calculated at $T_{\text {initial }}$ and $T_{\text {final }}$ and an average of the two was calculated and used in equation 29.

Next, the residual enthalpy necessary to convert the ideal-gas at its final temperature $\left(\mathrm{H}^{\mathrm{R}}{ }_{2}\right)$ back to a real gas is once again achieved by equation 28 . The result is the total enthalpy of a real vapor at a specific temperature and pressure. If the total liquid enthalpy is desired, simply subtract the enthalpy of vaporization at the stated equilibrium temperature. One suitable expression is the Clausius-Clapeyron equation:

$$
\Delta H=T \Delta V^{*} d P^{s a t} / d T
$$

However, approximations given by the Antoine equation in calculating the saturation pressure rendered the Clausius-Clapeyron equation unusable; at low pressures and high vapor molar volumes, the enthalpy of vaporization as calculated by equation 30 was greater than the enthalpy of vaporization at the nbp listed in Reklaitis (1983) - this is not possible. An appropriate expression used to calculate the enthalpy of vaporization more precisely is given by Watson (Smith et al., 2002)

$$
\Delta H_{2} / \Delta H_{1}=\left(1-T_{r 2} / 1-T_{r 1}\right)^{0.38}
$$

where $T_{r 2}$ is the reduced temperature at the stated equilibrium temperature, $T_{r 1}$ is the reduced temperature at the normal boiling point, and $\Delta \mathrm{H}_{1}$ is the enthalpy of vaporization at the normal boiling point given in Reklaitis (1983). The reduced temperature is defined by the expression (Smith et al., 2002): 


$$
T_{r}=T / T_{c}
$$

In summary, the total vapor and liquid enthalpies for a particular compound at a specified temperature and pressure are calculated by the following (Smith et al., 2002):

$$
\begin{gathered}
H_{\text {vapor }}=H^{0}+\Delta H_{\text {vap }, n}-H^{R}{ }_{1}+c_{p, \text { avg }}\left(T_{\text {final }}-T_{\text {initial }}\right)+H^{R} \\
H_{\text {liquid }}=H^{0}+\Delta H_{\text {vap }, n}-H^{R}{ }_{1}+c_{p, \text { avg }}\left(T_{\text {final }}-T_{\text {initial }}\right)+H^{R}{ }_{2}-\Delta H_{2}
\end{gathered}
$$

These vapor and liquid enthalpies, where applicable, were plotted along the $\mathrm{x}$-axis versus pressure, which was plotted along the y-axis on a logarithmic scale. 


\section{RESULTS AND DISCUSSION}

For each of the species studied, vapor-liquid equilibrium relationships and lines of constant temperature (called isotherms) were analyzed via the Reidlich-Kwong-Soave, Reidlich-Kwong, original van der Waals, and modified van der Waals equations of state. FIGURES 5 and 19 through 41 illustrate the Pv relationships for each of the six compounds. Similarly, FIGURES 6 and 42 through 58 show the PH relationships for the aforementioned substances. All of these figures are located in Appendices I and II. These diagrams also contain equilibrium and isothermal pressure-molar enthalpy relationships. An example of these charts is shown in FIGURES 5 and 6.
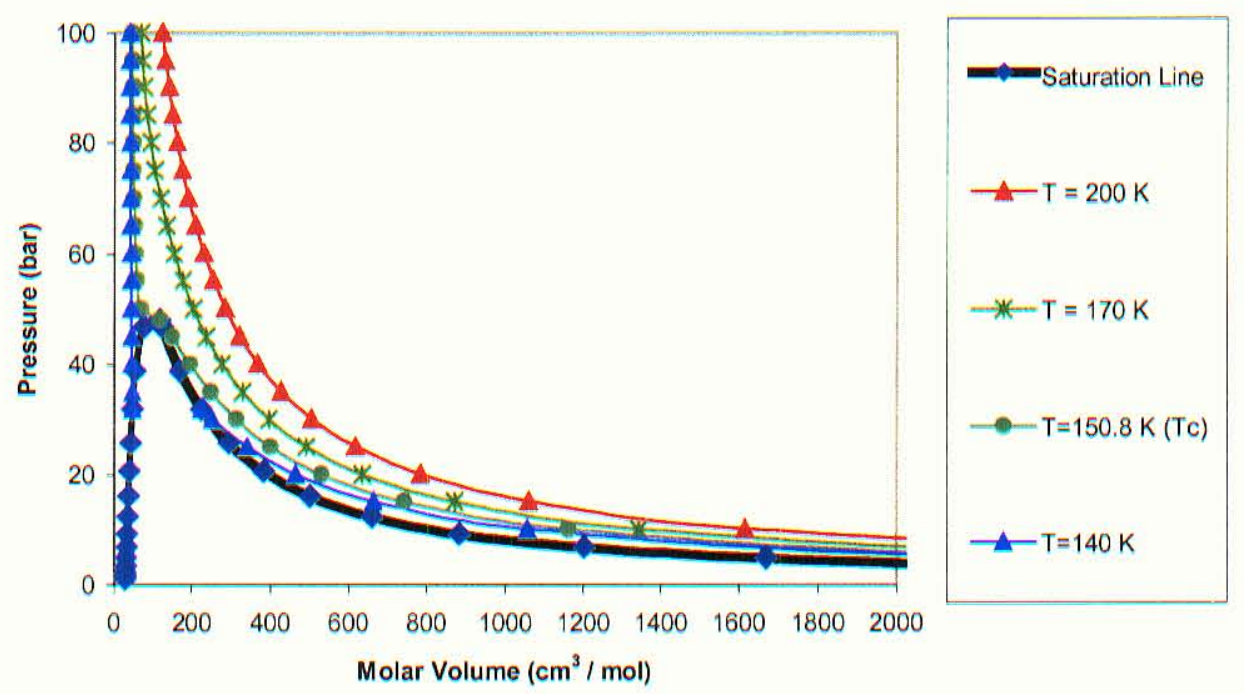

FIGURE 5 - Vapor and Liquid Molar Volumes of Argon Using the Reidlich-Kwong-Soave Equation of State 

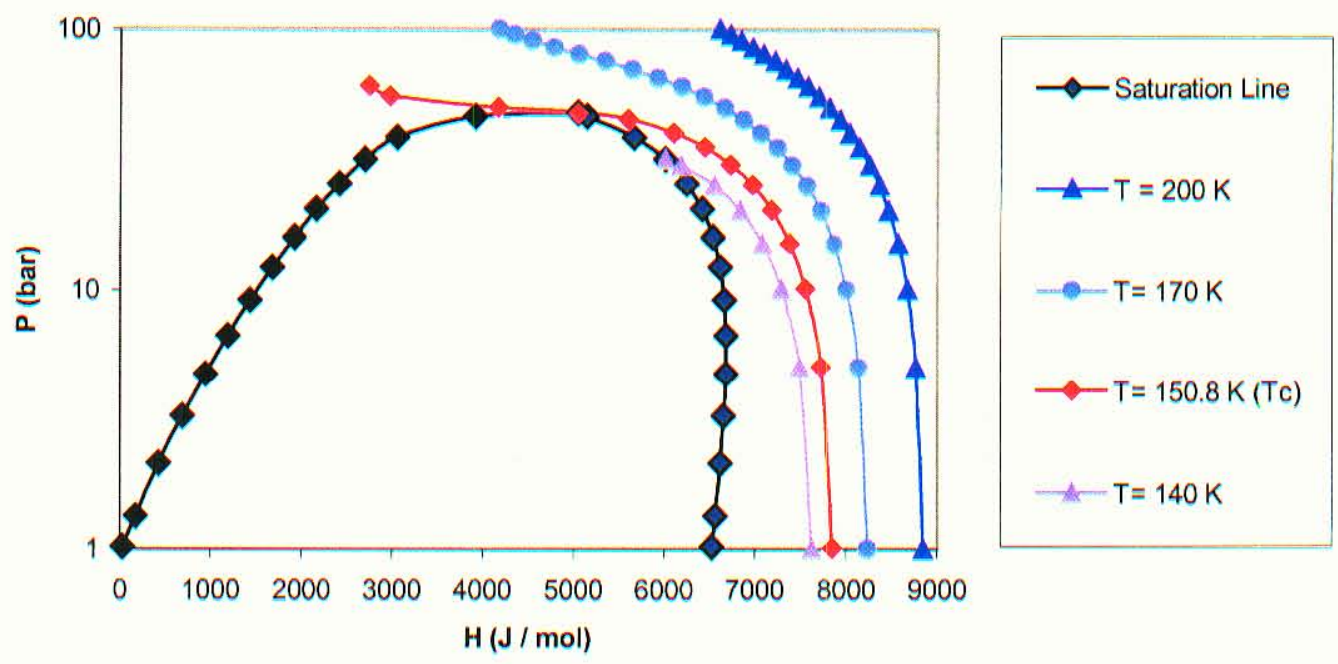

FIGURE 6 - Vapor and Liquid Molar Enthalpies of Argon Using the Reidlich-Kwong Equation of State

FIGURES 7 thru 12 depict pressure-molar volume relationships for the six different compounds analyzed in this experiment. Each of the figures contains the vapor-liquid equilibrium relationships as calculated per the Reidlich-Kwong-Soave, Reidlich-Kwong, original van der Waals, and modified van der Waals equations of state. These calculated values are compared alongside experimental vapor-liquid equilibrium data taken from Perry's Chemical Engineers' Handbook, Seventh Edition (1997) (note: no experimental data was available for $n$-pentane).

For argon, shown in FIGURE 7, the vapor molar volumes calculated by the modified van der Waals equation are higher than the vapor molar volumes as calculated by the original van der Waals equation. In turn, the original VDW predicted higher vapor molar 
volumes than the RK and RKS equations of state, the latter of which best agreed with the experimental data.

However, results were more promising when applied to the real gases (ethane, propane, $n$-butane, and $n$-pentane). The modified van der Waals equation predicted vapor molar volumes just as well as, or at times better than, the Reidlich-Kwong and Reidlich-Kwong-Soave equations of state. Calculated values were very near experimental data; an exception was $n$-butane, where some expected values were actually less than experimental values. For all four hydrocarbon gases, the modified van der Waals was much more precise than the original van der Waals equation of state. Furthermore, the modified van der Waals equation of state did well estimating the critical volume - it was often several orders of magnitude more precise than the other equations of state. These results are illustrated in FIGURES 8 through 11.

The inconsistency of the modified VDW is typified by the analysis of water at its vapor-liquid equilibrium. Calculated values of the vapor molar volumes are significantly higher for the modified van der Waals equation of state than for any of the other equations of state; the expected values fall nowhere near the experimental data. Furthermore, values for the liquid molar volumes of water would not even converge to a final answer, whereas for the other five compounds liquid molar volumes were consistent with given experimental data. The semi-uniqueness of water can be attributed to its intermolecular forces. Water is an extremely polar molecule; furthermore, hydrogen bonding produces significant attractive forces between water molecules. On the other hand, argon is a noble gas and possesses no intermolecular forces, and the four 
hydrocarbons are relatively nonpolar molecules that possess only insignificant inductive and dispersive intermolecular forces.
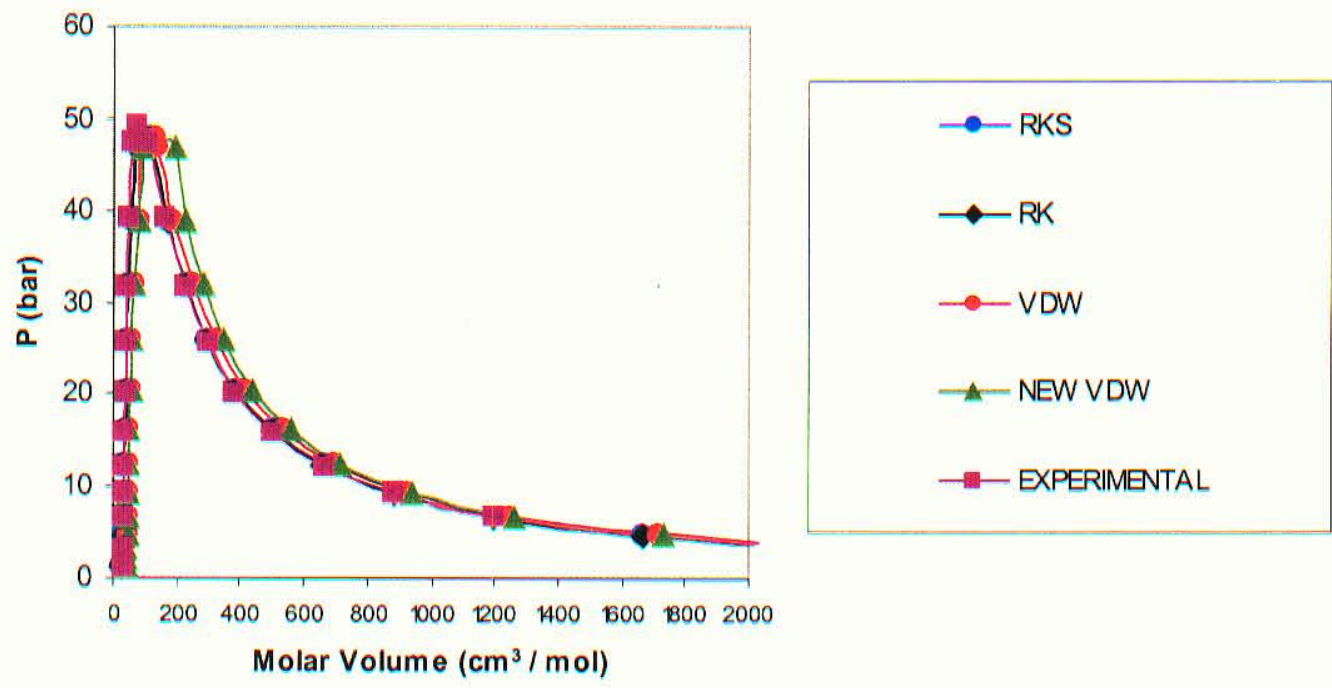

FIGURE 7 - Effect of Pressure on Argon Molar Volume

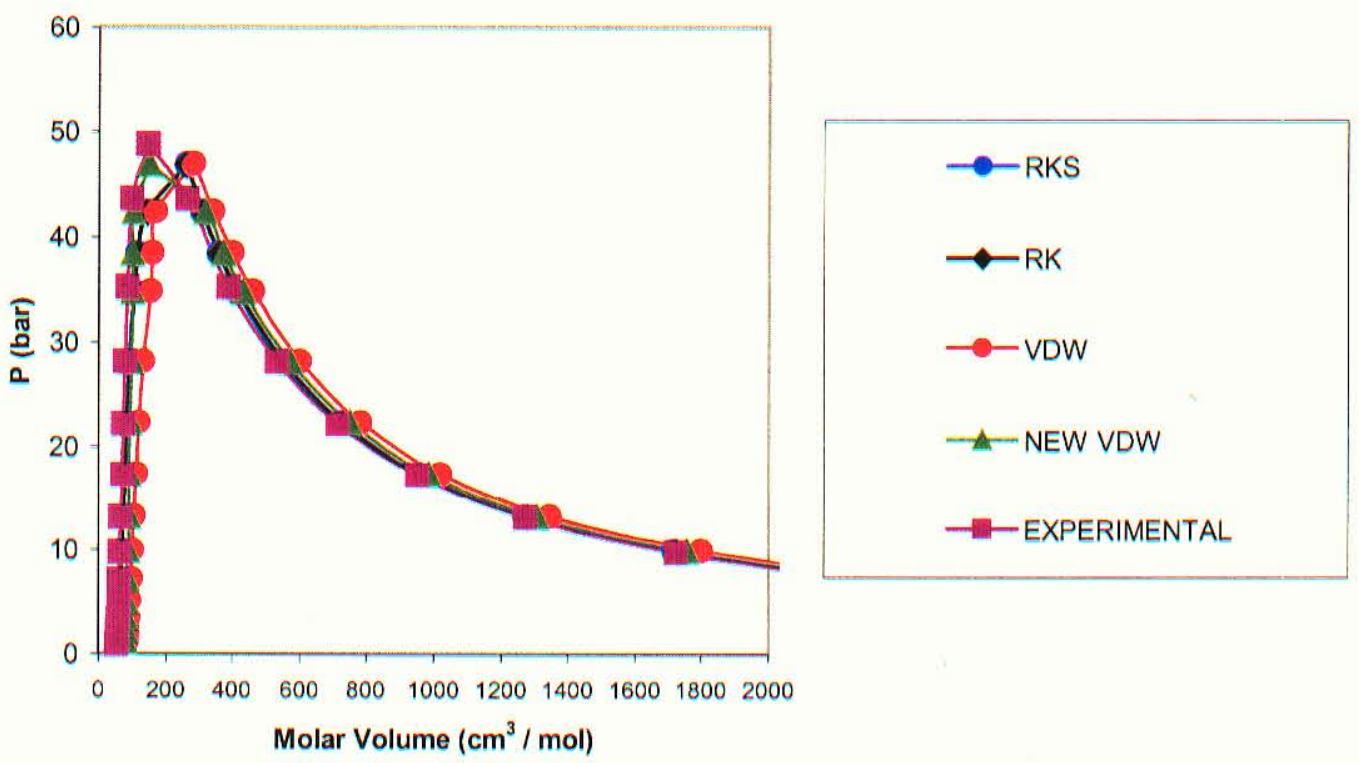

FIGURE 8 - Effect of Pressure on Ethane Molar Volume 


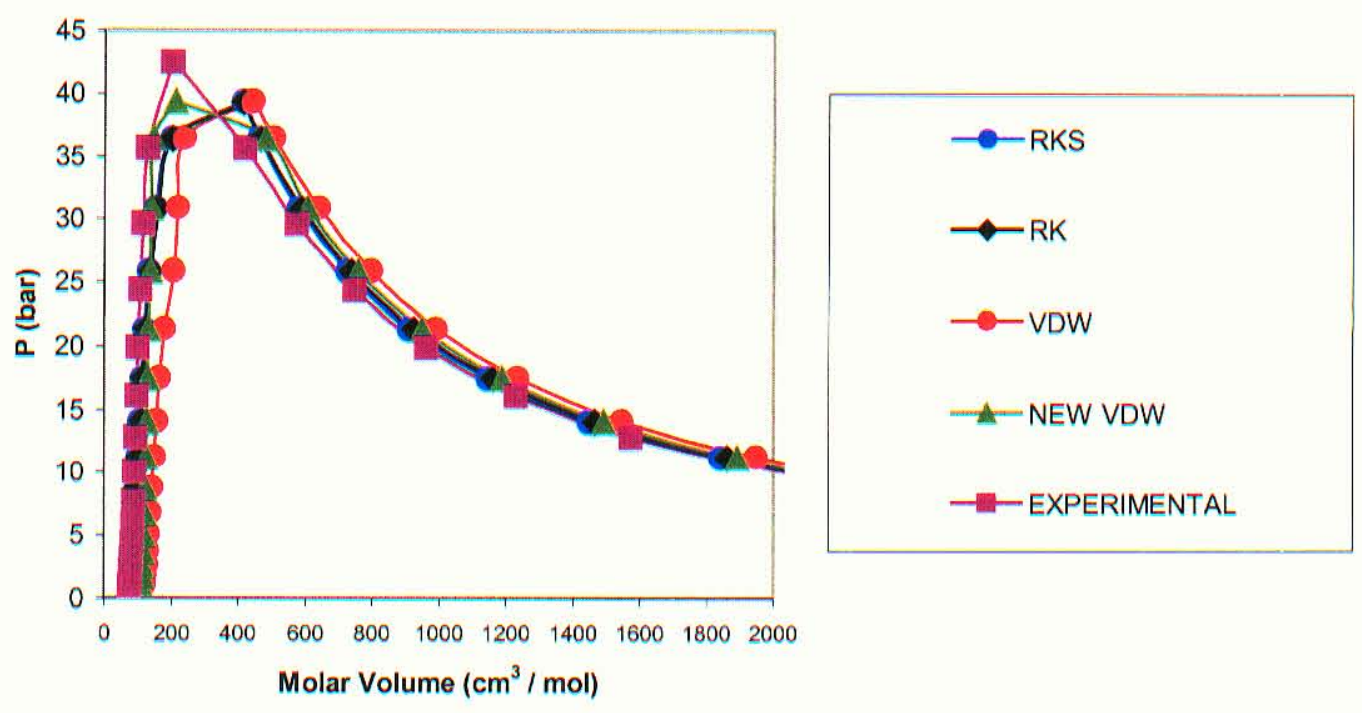

FIGURE 9 - Effect of Pressure on Propane Molar Volume
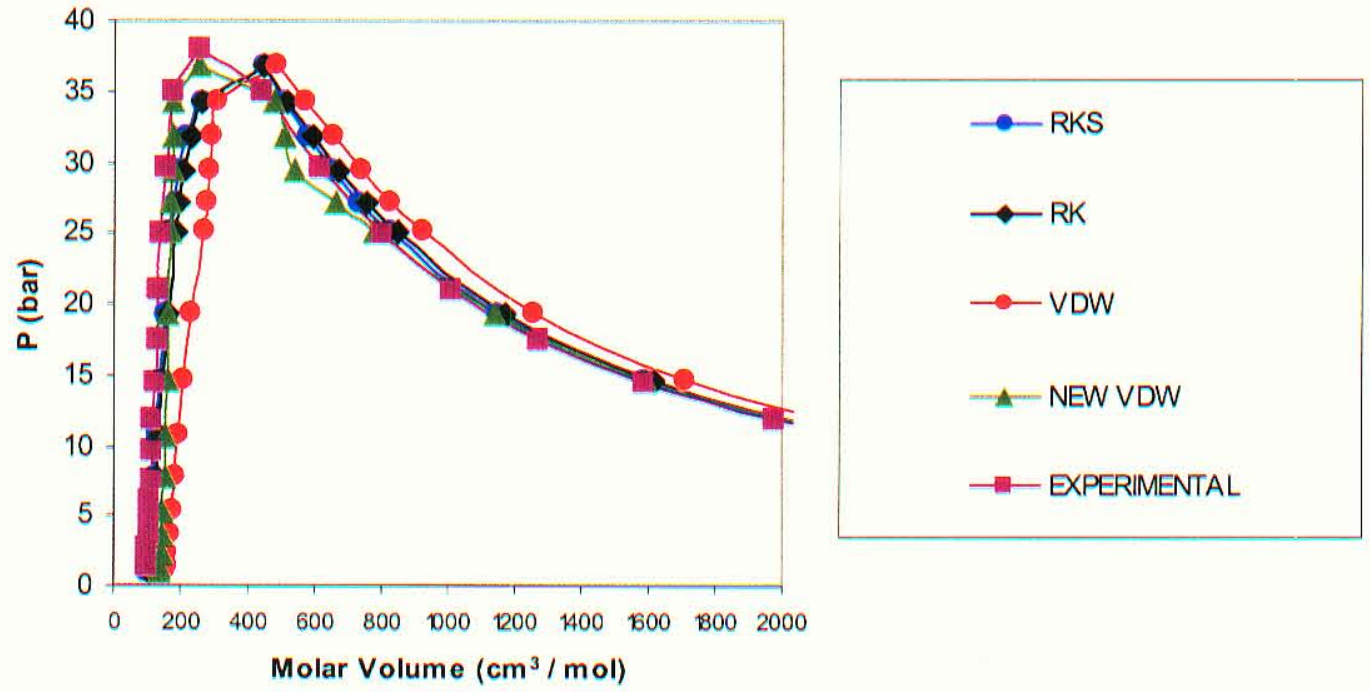

FIGURE 10 - Effect of Pressure on $n$-Butane Molar Volume 

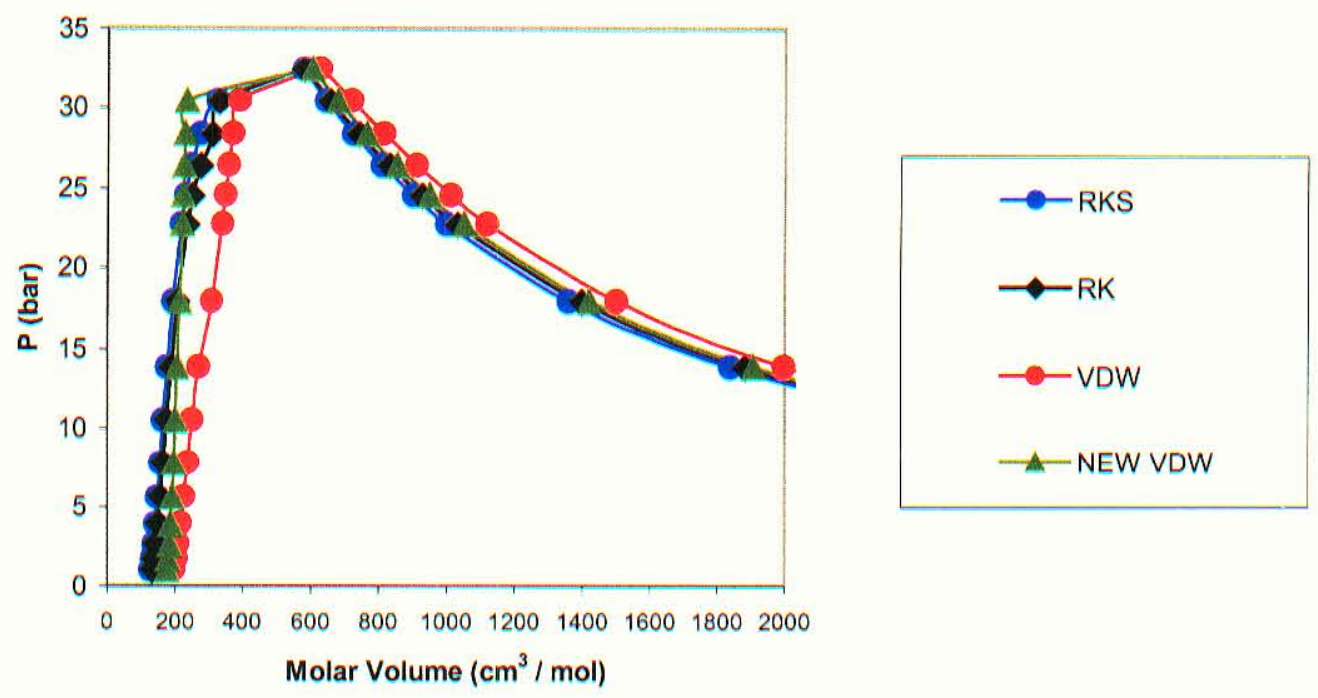

FIGURE 11 - Effect of Pressure on $n$-Pentane Molar Volume

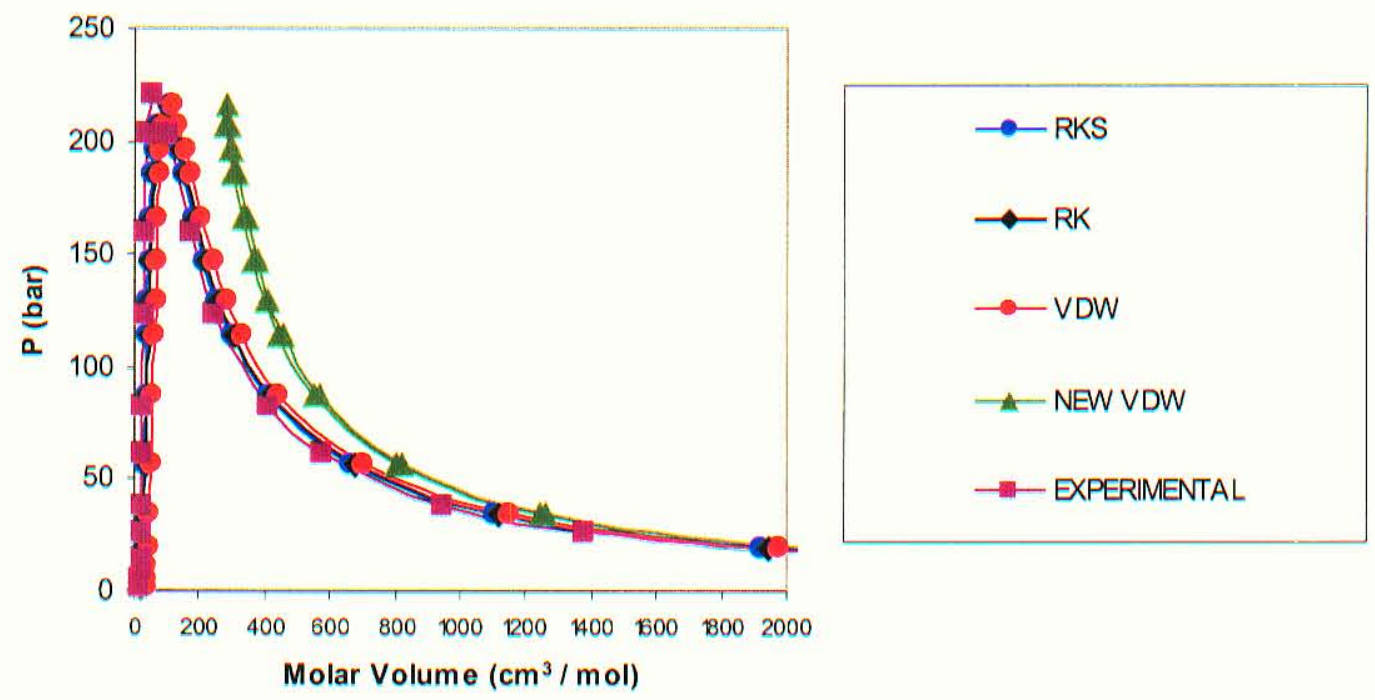

FIGURE 12 - Effect of Pressure on Water Molar Volume 
FIGURES 13 through 18 show the pressure-enthalpy relationships for the same six compounds. Analysis of these figures reveals that the Reidlich-Kwong equation of state more precisely predicts the total vapor and liquid molar enthalpies than the modified van der Waals equation of state; in many cases, analysis via the RK equation of state mimics the experimental data. In turn, the modified VDW is a better tool of predicting total molar enthalpies than the original van der Waals equation of state. The varying accuracies of the different equations of state are attributed to the residual enthalpies calculated via each equation of state. In other words, the residual enthalpy calculated by the modified van der Waals equation of state is much greater than the residual enthalpy as calculated by the original VDW; in addition, the residual enthalpy is much closer to the residual enthalpy as determined from the experimental data. In fact, for $n$-butane, the molar enthalpies as predicted by the modified VDW are very similar to the experimental enthalpies.

The lone exception once again is water. As illustrated in FIGURE 18, the total molar enthalpies as calculated by the modified van der Waals equation of state is far less accurate than the Reidlich-Kwong and original van der Waals equations of state. Furthermore, the data as predicted by the modified van der Waals is nowhere near the experimental data. 


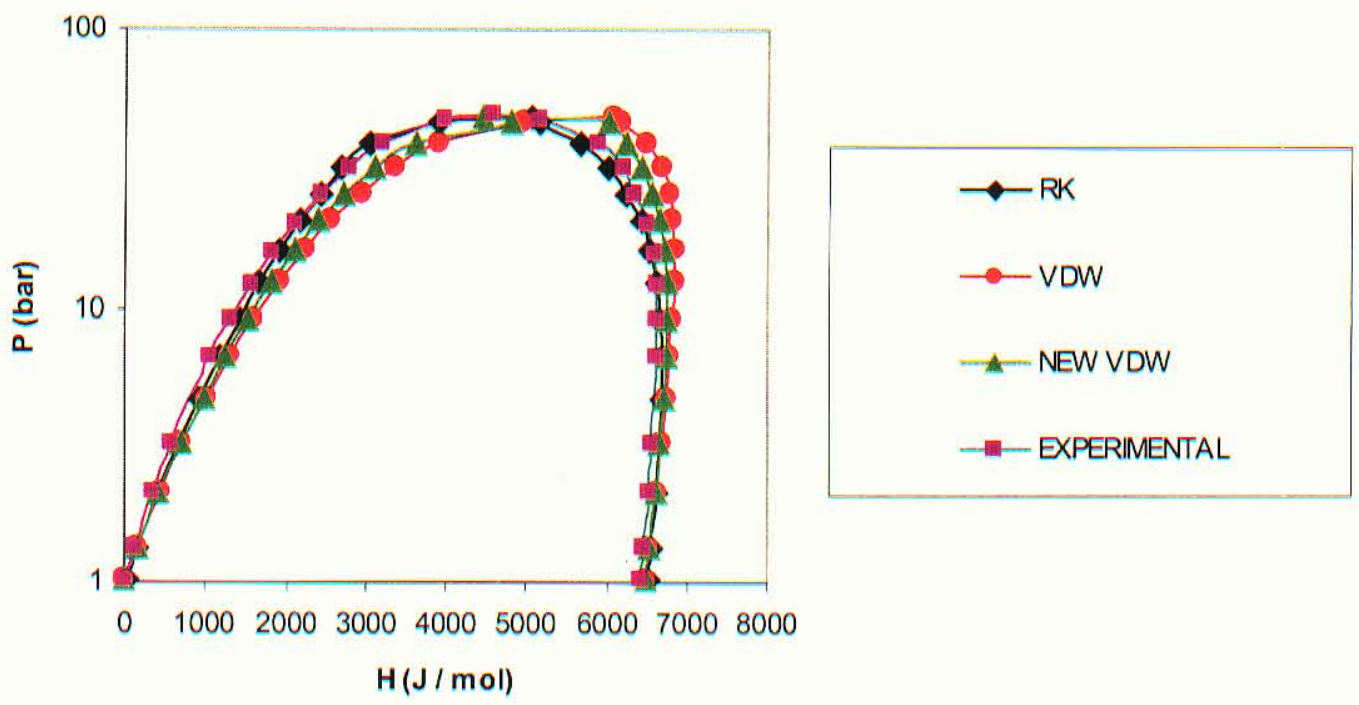

FIGURE 13 - Effect of Pressure on Argon Molar Enthalpy
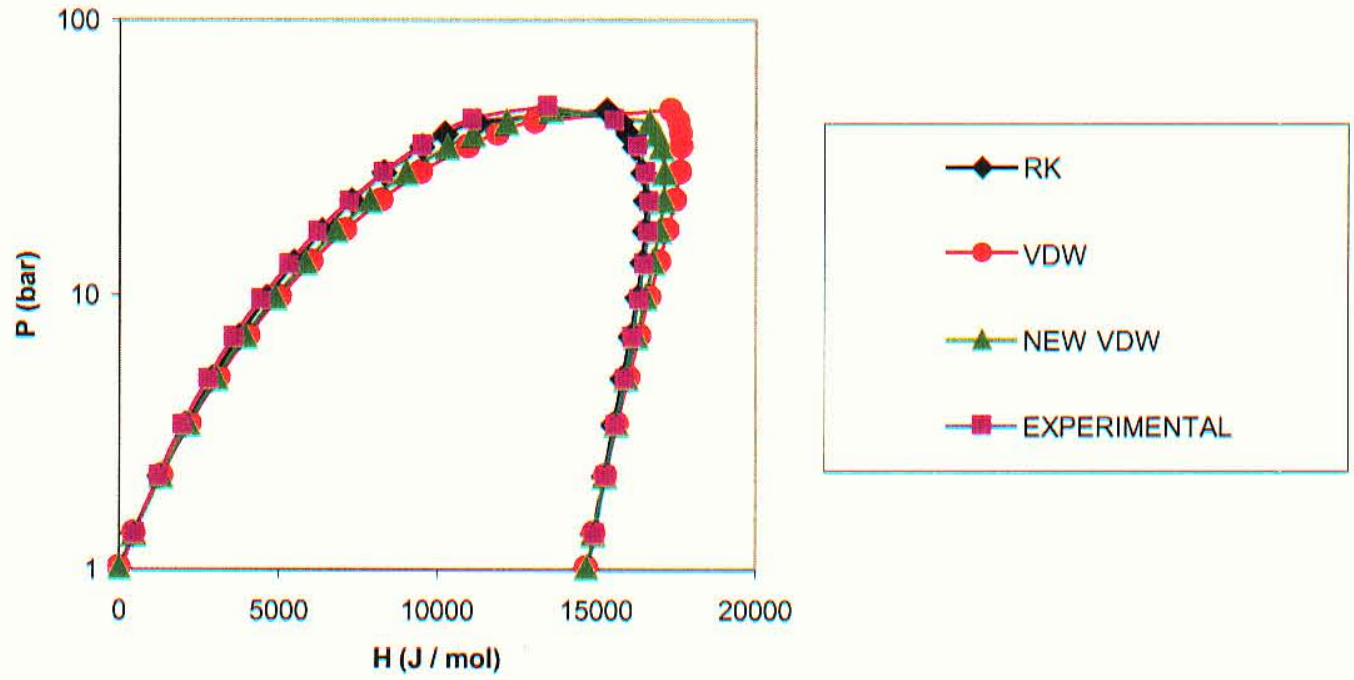

FIGURE 14 - Effect of Pressure on Ethane Molar Enthalpy 


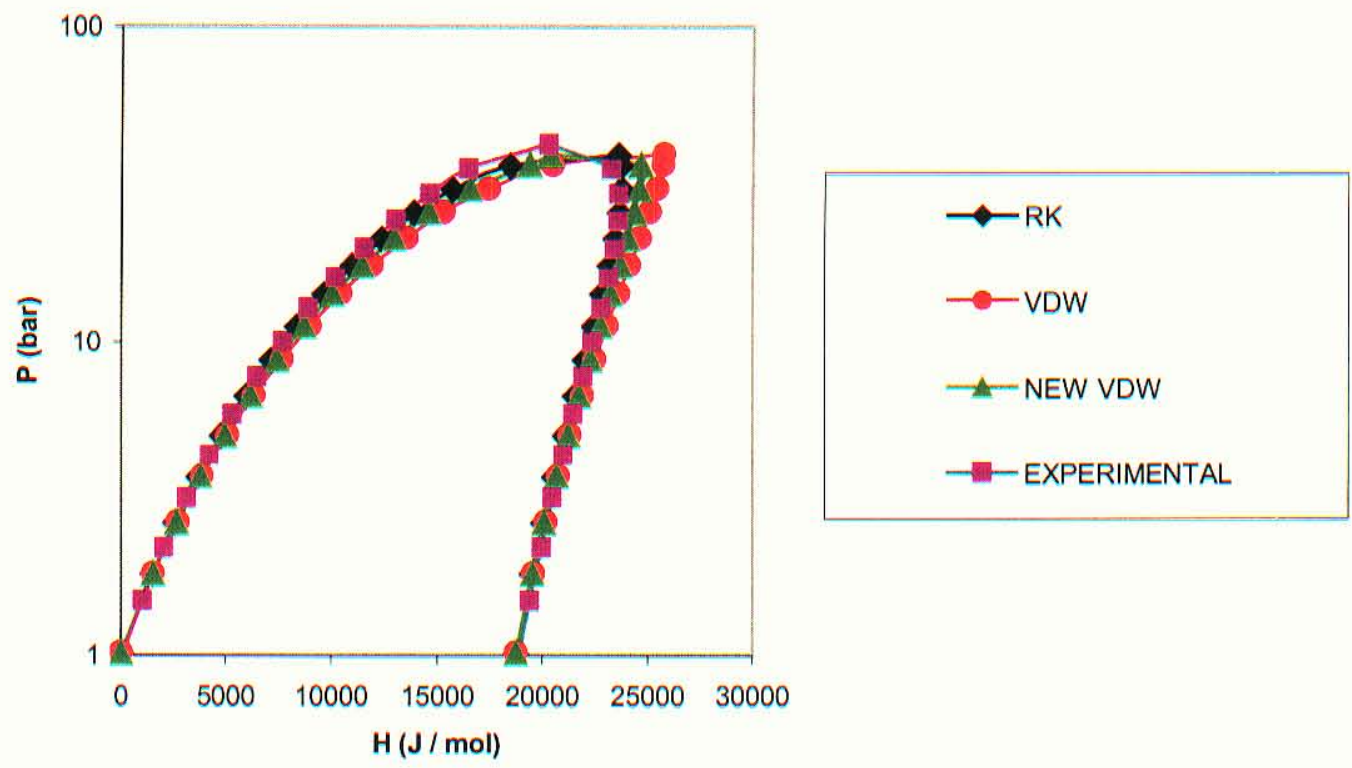

FIGURE 15 - Effect of Pressure on Propane Molar Enthalpy
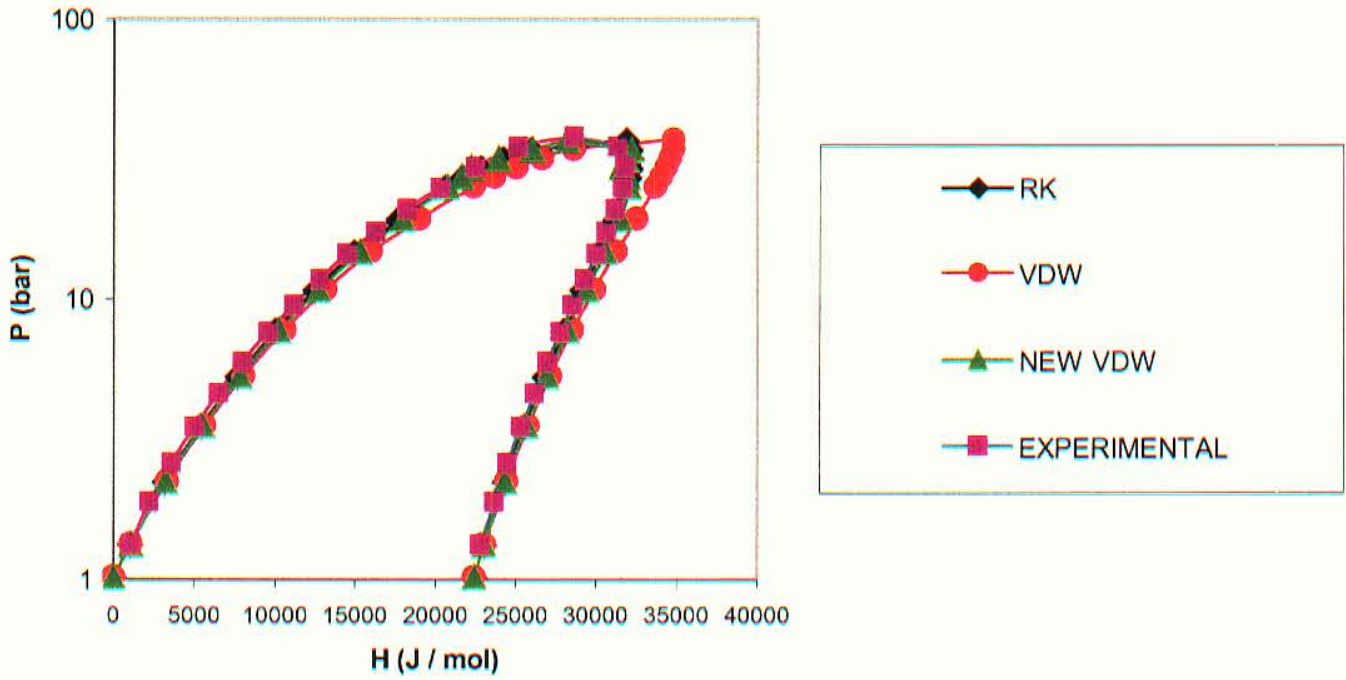

FIGURE 16 - Effect of Pressure on $n$-Butane Molar Enthalpy 

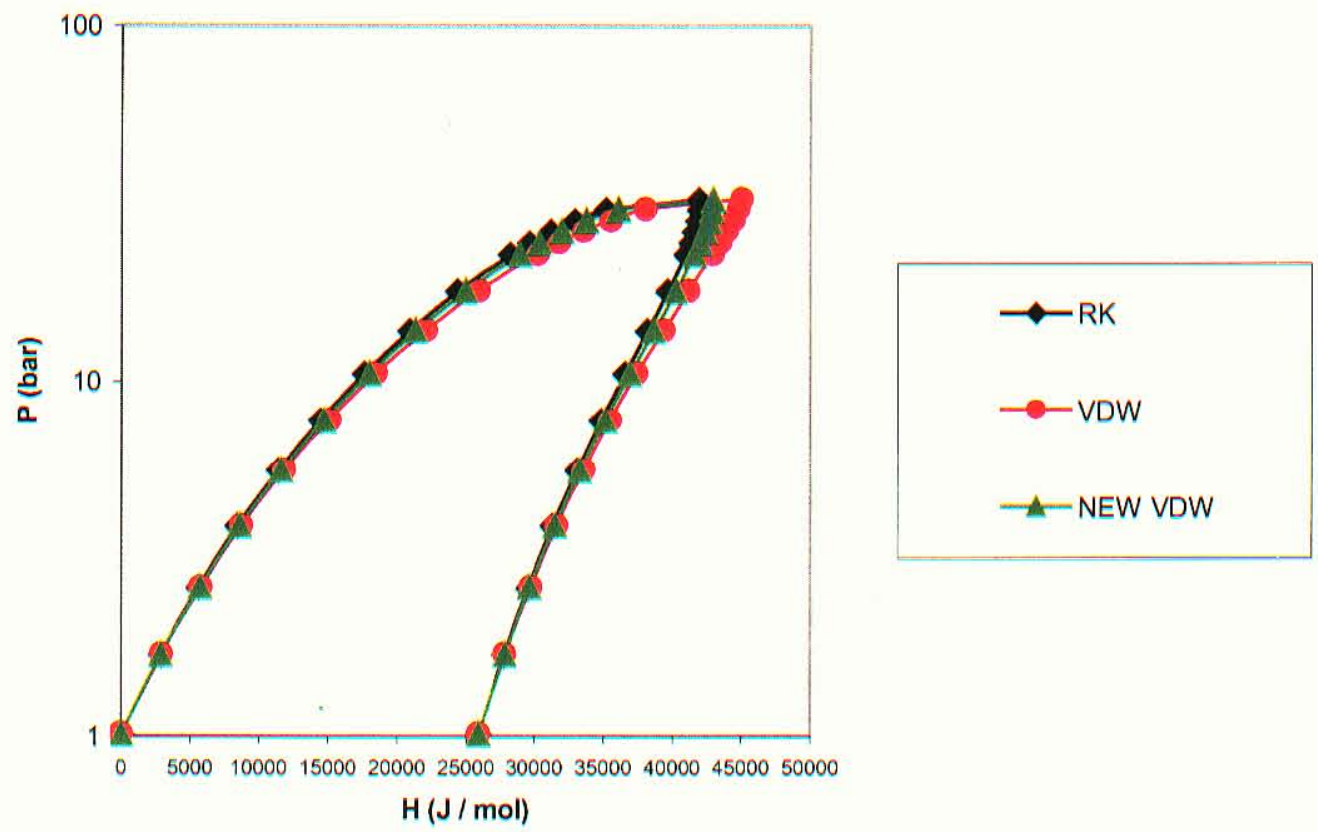

FIGURE 17 - Effect of Pressure on $n$-Pentane Molar Enthalpy

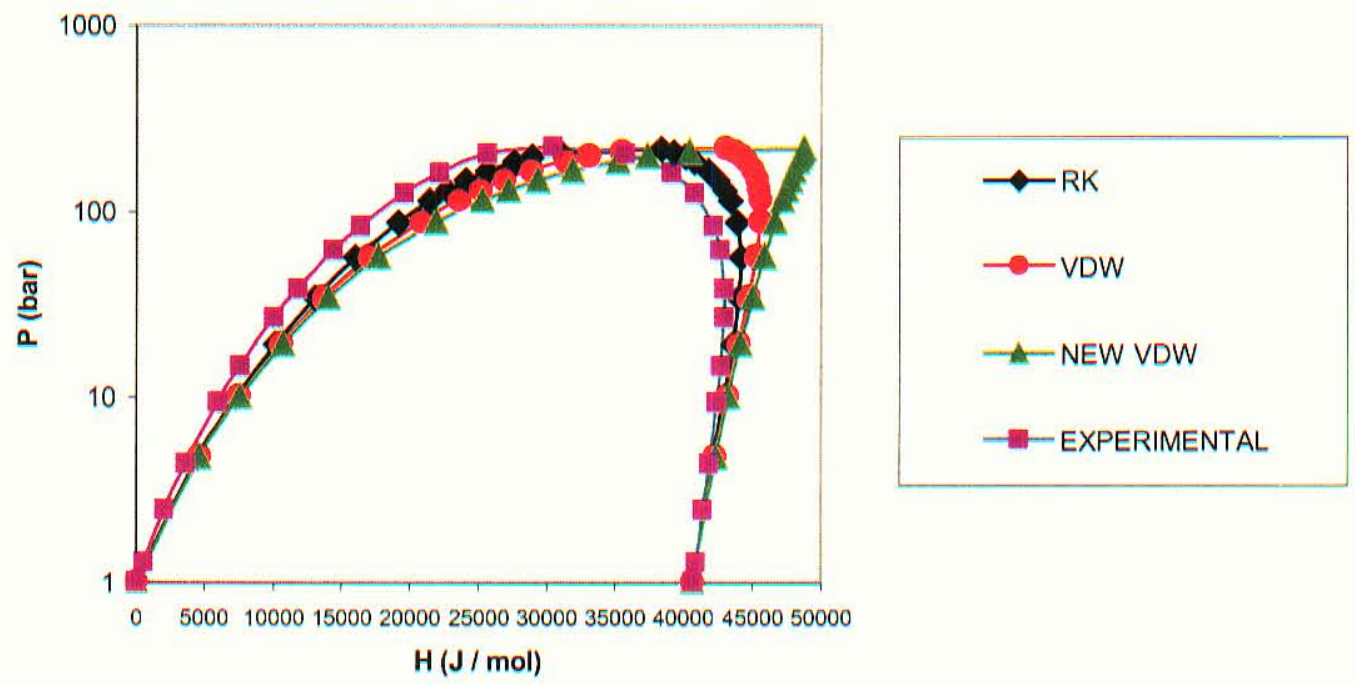

FIGURE 18 - Effect of Pressure on Water Molar Enthalpy 
The magnitude of the value of the residual enthalpy is dependent upon the equation of state that is utilized to calculate the residual enthalpy. Each equation of state calculates the $a$ and $b$ parameters by different methods. The dependence of these parameters upon changes in temperature $(T)$ and molar volume $(v)$ affects the relative magnitude of the residual enthalpy. For example, the modified van der Waals equation of state calculates the residual enthalpy by the following (McNeill et al., 2004):

$$
\begin{gathered}
H^{R}=R T\left[b_{H S} /\left(v-b_{H S}\right)\right]+\left[\left(a_{2} / v\right)^{*}\left(T / T_{c}\right)\right]-2 a_{1} / v \\
b_{H S}=\left(b_{0} / 4\right) *\left[1+\sqrt{1+2 b_{0}^{2}\left(\rho-2 / b_{0}\right)^{2}}\right] \\
a_{H S}=a_{1}-a_{2}\left(T / T_{c}\right)
\end{gathered}
$$

TABLES X AND XI illustrate how the residual enthalpy changes for ethane as one of the modified van der Waals parameters is varied while keeping the other constant.

\section{TABLE X}

EFFECT OF $a$-PARAMETER ON ETHANE RESIDUAL ENTHALPY

\begin{tabular}{|c|c|c|c|c|}
\hline $\mathbf{T}(\mathbf{K})$ & $\mathbf{P}_{\text {sat }}(\mathbf{b a r})$ & $\mathbf{a}_{\mathrm{HS}}\left(\mathbf{b a r}^{*} \mathbf{c m}^{6} / \mathbf{m o l}^{2}\right)$ & $\mathbf{b}_{\mathrm{HS}}\left(\mathbf{c m}^{\mathbf{3}} / \mathbf{m o l}\right)$ & $\mathbf{H}_{2}^{\mathbf{R}}(\mathbf{J} / \mathbf{m o l})$ \\
\hline 360 & 1.0 & $6.298 \mathrm{E}+06$ & 93.64 & -37.5 \\
\hline 350 & 1.0 & $6.337 \mathrm{E}+06$ & 93.64 & -39.0 \\
\hline 340 & 1.0 & $6.376 \mathrm{E}+06$ & 93.63 & -40.6 \\
\hline 330 & 1.0 & $6.414 \mathrm{E}+06$ & 93.63 & -42.3 \\
\hline 320 & 1.0 & $6.453 \mathrm{E}+06$ & 93.62 & -44.1 \\
\hline 310 & 1.0 & $6.492 \mathrm{E}+06$ & 93.62 & -46.0 \\
\hline 300 & 1.0 & $6.531 \mathrm{E}+06$ & 93.62 & -48.0 \\
\hline 290 & 1.0 & $6.569 \mathrm{E}+06$ & 93.62 & -50.2 \\
\hline 280 & 1.0 & $6.608 \mathrm{E}+06$ & 93.61 & -52.5 \\
\hline 270 & 1.0 & $6.647 \mathrm{E}+06$ & 93.61 & -55.0 \\
\hline 260 & 1.0 & $6.686 \mathrm{E}+06$ & 93.61 & -57.7 \\
\hline
\end{tabular}




\section{TABLE XI}

\section{EFFECT OF $b$-PARAMETER ON ETHANE RESIDUAL ENTHALPY}

\begin{tabular}{|c|c|c|c|c|}
\hline $\mathbf{T}(\mathbf{K})$ & $\mathbf{P}_{\text {sat }}$ (bar) & $\mathbf{a}_{\mathrm{HS}}\left(\mathbf{b a r}^{*} \mathbf{c m}^{6} / \mathbf{m o l}^{2}\right)$ & $\mathbf{b}_{\mathrm{HS}}\left(\mathbf{c m}^{3} / \mathbf{m o l}\right)$ & $\mathbf{H}^{\mathbf{R}}{ }_{\mathbf{2}}(\mathbf{J} / \mathbf{m o l})$ \\
\hline 360 & 1.0 & $6.298 \mathrm{E}+06$ & 93.64 & -37.5 \\
\hline 360 & 5.0 & $6.298 \mathrm{E}+06$ & 93.24 & -190.1 \\
\hline 360 & 10.0 & $6.298 \mathrm{E}+06$ & 92.73 & -387.1 \\
\hline 360 & 15.0 & $6.298 \mathrm{E}+06$ & 92.18 & -591.6 \\
\hline 360 & 20.0 & $6.298 \mathrm{E}+06$ & 91.62 & -804.3 \\
\hline 360 & 25.0 & $6.298 \mathrm{E}+06$ & 91.02 & -1026.1 \\
\hline 360 & 30.0 & $6.298 \mathrm{E}+06$ & 90.40 & -1257.9 \\
\hline 360 & 35.0 & $6.298 \mathrm{E}+06$ & 89.74 & -1500.7 \\
\hline 360 & 40.0 & $6.298 \mathrm{E}+06$ & 89.04 & -1755.5 \\
\hline 360 & 45.0 & $6.298 \mathrm{E}+06$ & 88.30 & -2023.7 \\
\hline 360 & 50.0 & $6.298 \mathrm{E}+06$ & 87.51 & -2306.4 \\
\hline 360 & 55.0 & $6.298 \mathrm{E}+06$ & 86.67 & -2604.6 \\
\hline 360 & 60.0 & $6.298 \mathrm{E}+06$ & 85.77 & -2919.0 \\
\hline 360 & 65.0 & $6.298 \mathrm{E}+06$ & 84.81 & -3249.2 \\
\hline 360 & 70.0 & $6.298 \mathrm{E}+06$ & 83.80 & -3593.4 \\
\hline 360 & 75.0 & $6.298 \mathrm{E}+06$ & 82.73 & -3947.5 \\
\hline 360 & 80.0 & $6.298 \mathrm{E}+06$ & 81.64 & -4304.4 \\
\hline 360 & 85.0 & $6.298 \mathrm{E}+06$ & 80.54 & -4654.8 \\
\hline 360 & 90.0 & $6.298 \mathrm{E}+06$ & 79.46 & -4989.3 \\
\hline 360 & 95.0 & $6.298 \mathrm{E}+06$ & 78.44 & -5300.1 \\
\hline 360 & 100.0 & $6.298 \mathrm{E}+06$ & 72.76 & -6332.2 \\
\hline
\end{tabular}

As pressure is increased while temperature is kept constant, the $a$-parameter remains unchanged. However, the molar volume ( $v)$ and $b$-parameter decrease; the magnitude of the decrease of $v$ is much greater then the change in $b$ as the pressure is increased.

Therefore, the quantity $b /(v-b)$ increases greatly because the quantity $(\nu-b)$ decreases significantly; as a result, the magnitude of the residual enthalpy $\left(\mathrm{H}^{\mathrm{R}}\right)$ increases by an extremely large amount.

The $b$-parameter remains essentially constant when pressure is kept constant; this keeps the molar volume $(v)$ relatively constant as well because the gas density $(\rho)$ does not change at constant pressure. The $a$-parameter changes as temperature changes, based on equation 14; the $b$-parameter also changes minimally (a temperature difference of 100 
degrees Kelvin causes a change in $b_{H S}$ by 0.03 ). Therefore, the only values that change the residual enthalpy at constant $b_{H S}$ is the temperature shown in the first two terms of equation 34. Unless the temperature changes significantly (on the order of magnitude of ten, for example), the residual enthalpy will not change significantly.

These assumptions are backed by data created from the modified van der Waals equation of state shown in TABLES X AND XI. While $a_{H S}$ is kept constant, the pressure is varied such that $b_{H S}$ decreases from approximately 94 to 73 . However, $H^{\mathrm{R}}$ increases by almost a factor of 200 . On the other hand, a temperature variation of 100 degrees $\mathrm{K}$ does not even affect the residual enthalpy by a factor of two. It is evident that the $b$-parameter influences the residual enthalpy more than the $a$-parameter. 


\section{CONCLUSIONS}

- Vapor molar volumes predicted by the modified van der Waals equation of state were less accurate than the Reidlich-Kwong-Soave, Reidlich-Kwong, and original van der Waals equations of state with respect to argon.

- Vapor molar volumes predicted by the modified van der Waals equation of state were more accurate then the original van der Waals equation of state when the hydrocarbons (ethane, propane, $n$-butane, and $n$-propane) were analyzed.

- Vapor molar volumes predicted by the modified van der Waals equation of state were just as accurate as the RKS and RK equations of state and also compared favorably with the experimental data.

- Critical volumes predicted by the modified van der Waals equation of state were consistent with experimentally determined critical volumes for all compounds except water.

- Liquid molar volumes predicted by the modified van der Waals equation of state mirrored experimentally measured liquid molar volumes for all compounds except water.

- All molar volumes predicted by the modified van der Waals equation of state were extremely inaccurate for water because of the relative proximity of the $a$-parameters, $a_{1}$ and $a_{2}$.

- Residual enthalpies predicted by the modified van der Waals equation of state were more accurate (i.e. closer to the experimental data) than residual enthalpies predicted by the original VDW equation of state for all of the compounds except water. 
- Residual enthalpies predicted by the modified van der Waals equation of state were less accurate (i.e. further from the experimental data) than residual enthalpies predicted by the Reidlich-Kwong equation of state for all six compounds.

- Residual enthalpies predicted by the modified van der Waals equation of state were less accurate than residual enthalpies predicted by the original VDW equation of state for water.

- The inaccuracy of the enthalpy data for water is also attributed to the relative proximity of the $a$-parameters for water.

- The $b$-parameter used in the modified van der Waals equation of state has a greater influence on the magnitude of the residual enthalpy than the $a$-parameter.

- The modified van der Waals equation of state is not thermodynamically consistent; in other words, different thermodynamic properties do not follow the same trends when compared to other equations of state for various compounds. 


\section{RECOMMENDATIONS}

- Apply the modified van der Waals equation of state to other compounds to determine if other trends can be discovered.

- Analyze thermodynamic properties, besides molar volume and molar enthalpy, using modified van der Waals equation of state to verify that the equation of state is thermodynamically inconsistent.

- Re-examine the values of $a_{1}$ and $a_{2}$ for water to ensure that these values are correct. 


\section{LIST OF REFERENCES}

Collins, D.J. 2003. Private Communication

Himmelblau, D.M. 2000. Basic Principles and Calculations in Chemical Engineering. Sixth Edition. Englewood Cliffs, NJ: Prentice-Hall.

McNeill, J.H. and Collins, D.J. 2004. Enhancement of the van der Waals Equation of State via Molecular Dynamics Simulations and the Hard-Sphere Model. (not published)

McNeill, J.H. and Collins, D.J. 2004. Hard-Sphere Simulation Results Applied to the van der Waals Gas Equation of State. (not published)

Prausnitz, J.M., Lichtenthaler, R.N., and de Azevedo, E.G. 1999. Molecular Thermodynamics of Fluid-Phase Equilibria. Third Edition. Upper Saddle River, NJ: Prentice-Hall.

Perry, R.H., et al. 1996. Perry's Chemical Engineers' Handbook. Seventh Edition. McGraw-Hill.

Reklaitis, G.V. 1983. Introduction to Material and Energy Balances. Wiley Press.

Smith, J.M., Van Ness, H.C., and Abbott, M.M. 1996. Introduction to Chemical Engineering Thermodynamics. Fifth Edition. McGraw-Hill.

Smith, J.M., Van Ness, H.C., and Abbott, M.M. 2002. Introduction to Chemical Engineering Thermodynamics. Sixth Edition. McGraw-Hill. 


\section{APPENDIX I}

PRESSURE-MOLAR VOLUME GRAPHS 


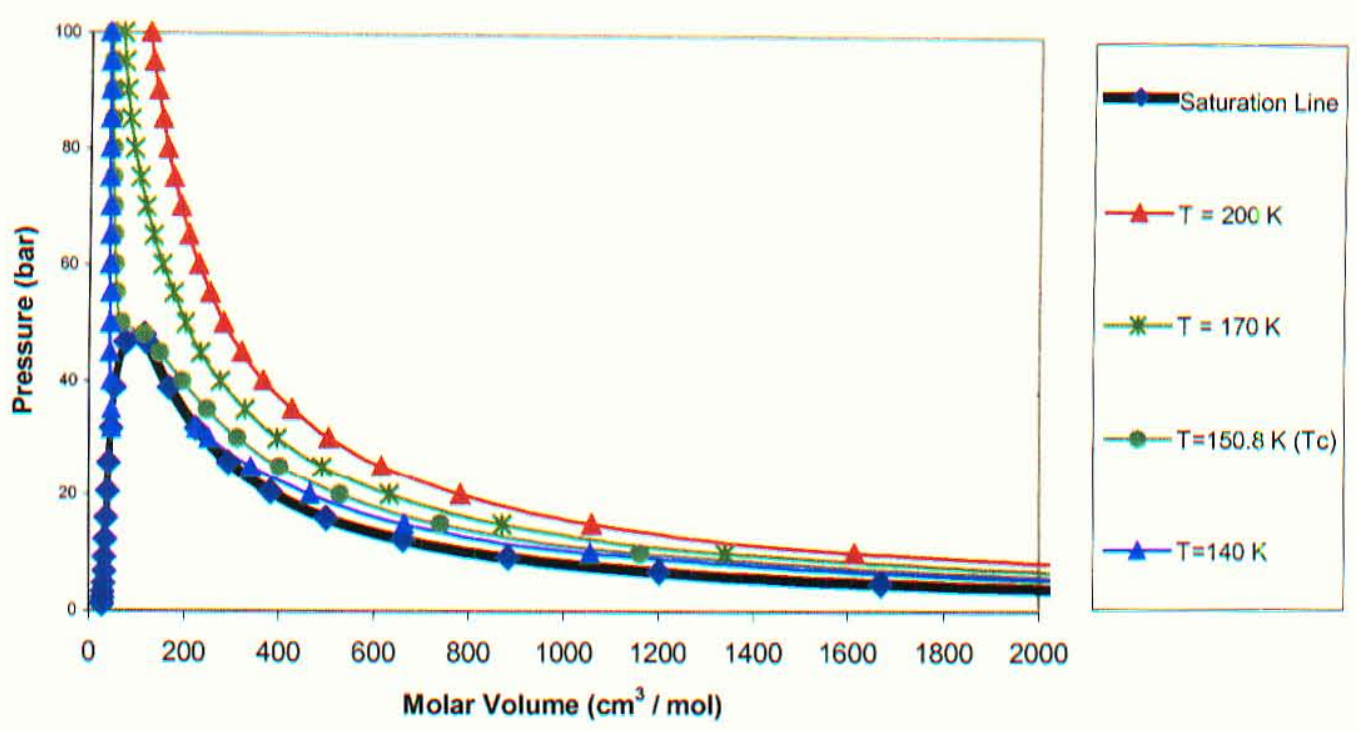

FIGURE 5 - Vapor and Liquid Molar Volumes of Argon Using the

Reidlich-Kwong-Soave Equation of State
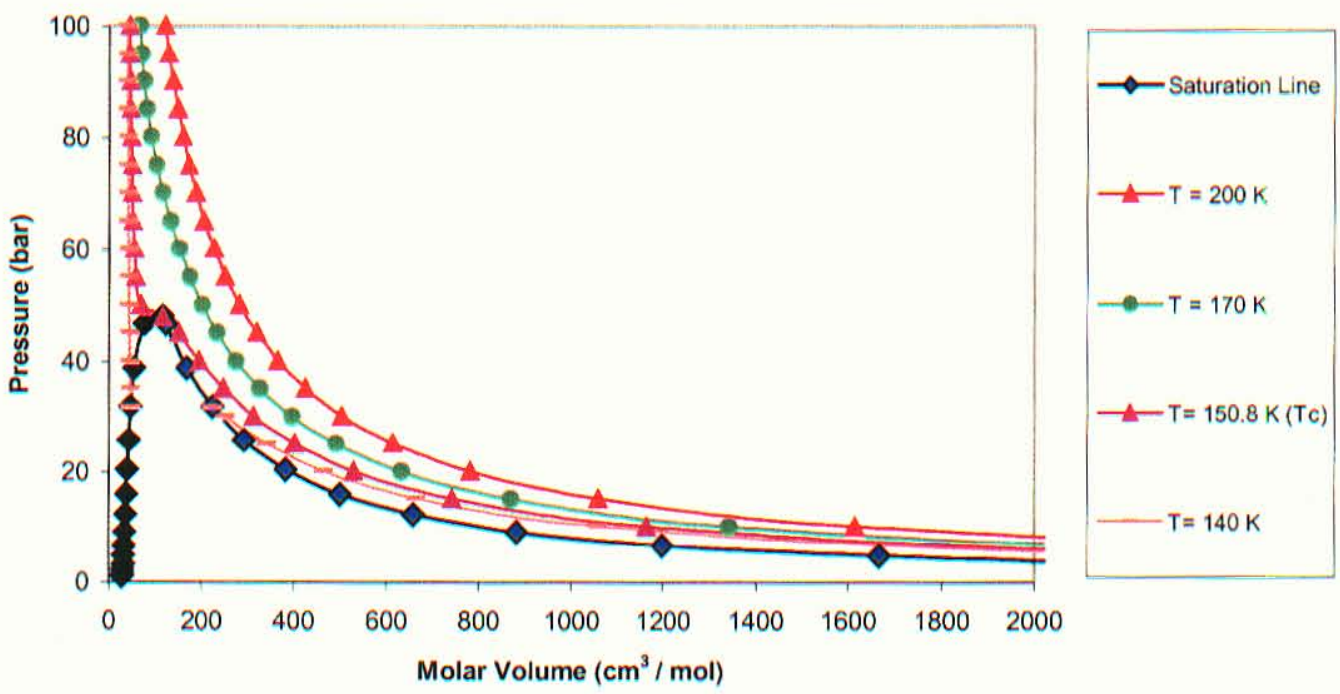

FIGURE 19 - Vapor and Liquid Molar Volumes of Argon Using the Reidlich-Kwong Equation of State 


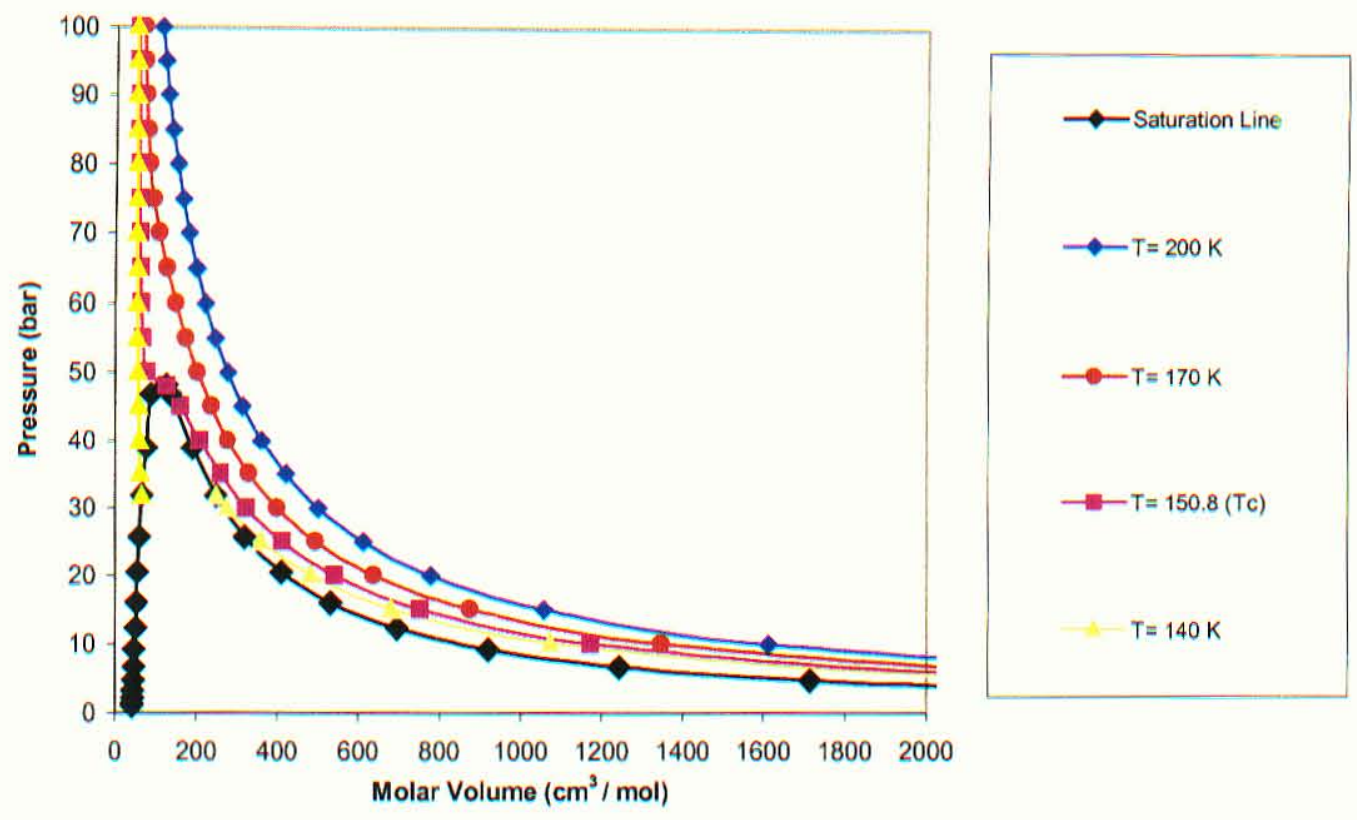

FIGURE 20 - Vapor and Liquid Molar Volumes of Argon Using the

Original van der Waals Equation of State
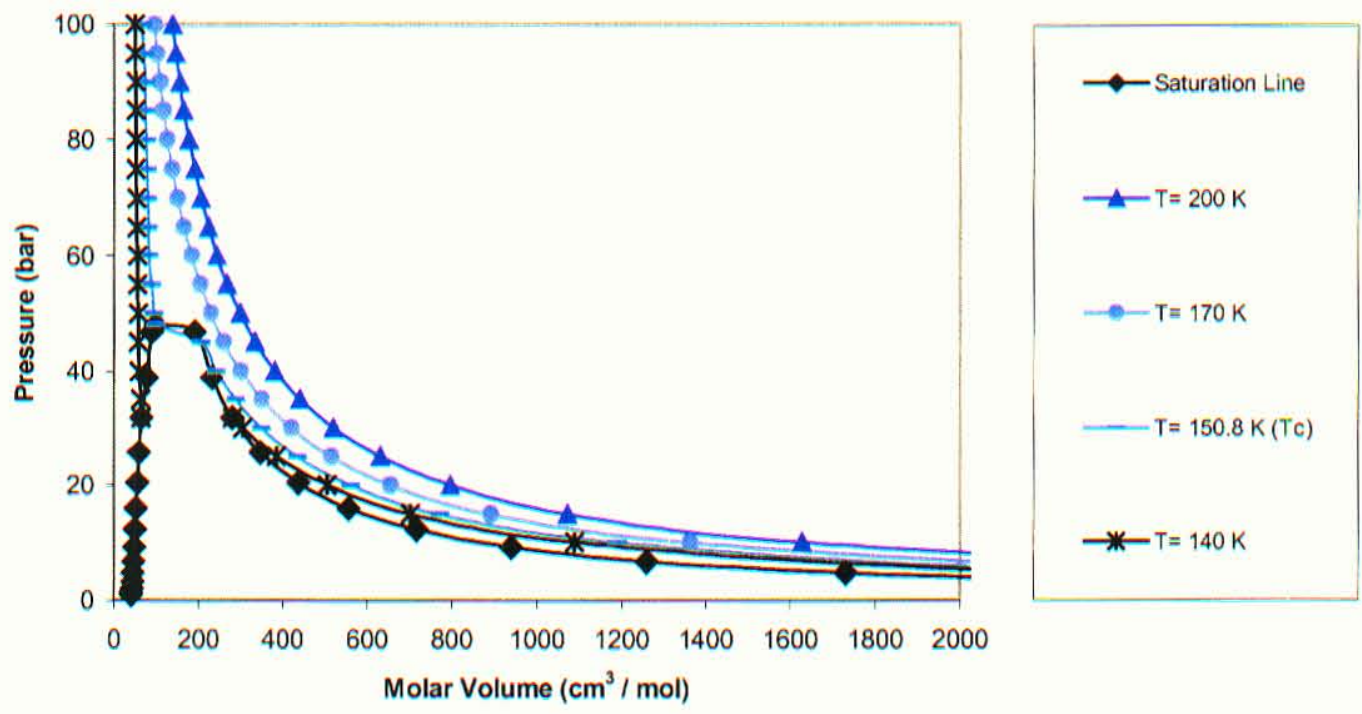

FIGURE 21 - Vapor and Liquid Molar Volumes of Argon Using the

Modified van der Waals Equation of State 

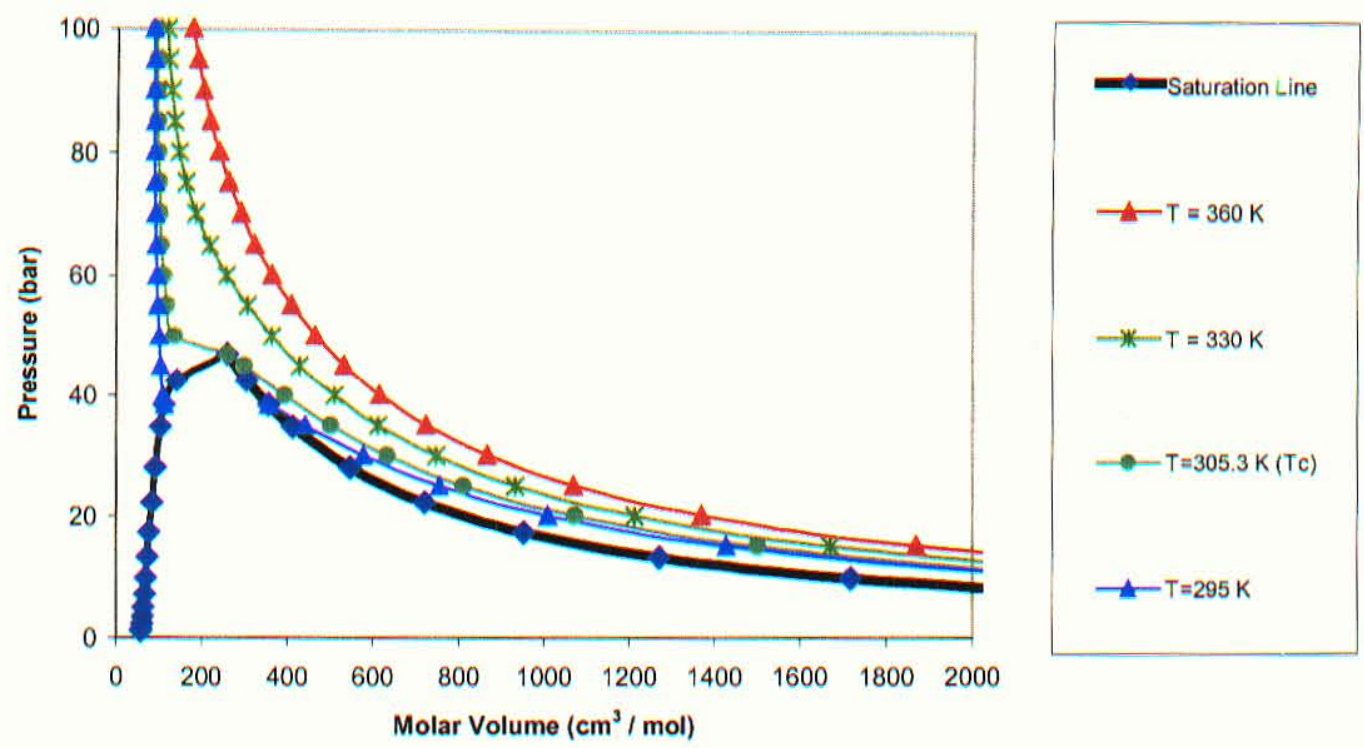

FIGURE 22 - Vapor and Liquid Molar Volumes of Ethane Using the

Reidlich-Kwong-Soave Equation of State
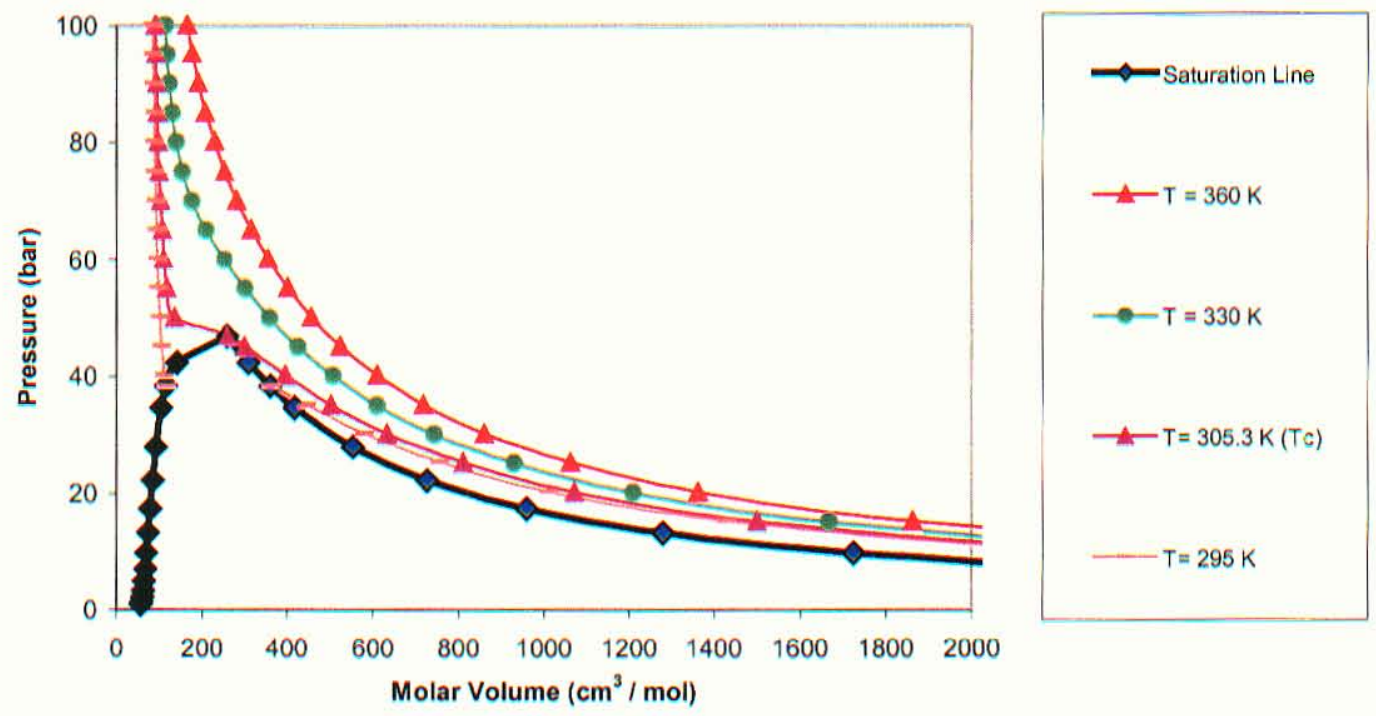

FIGURE 23 - Vapor and Liquid Molar Volumes of Ethane Using the

Reidlich-Kwong Equation of State 

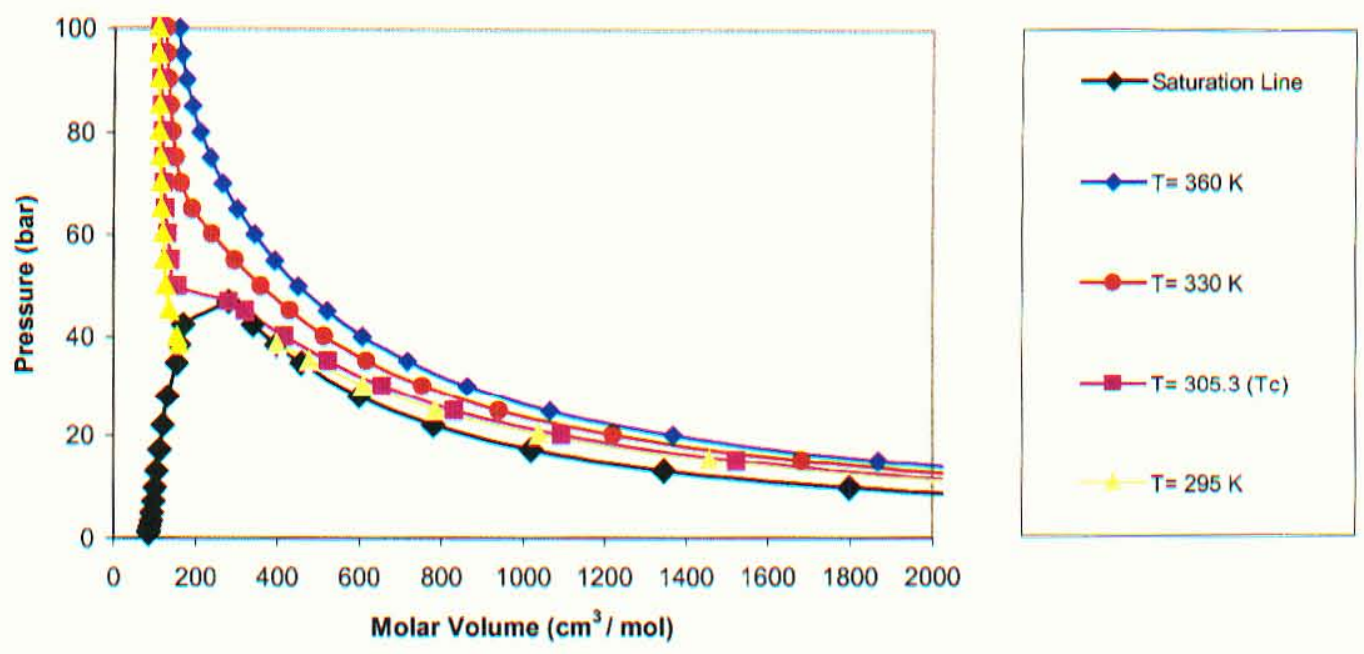

FIGURE 24 - Vapor and Liquid Molar Volumes of Ethane Using the

Original van der Waals Equation of State
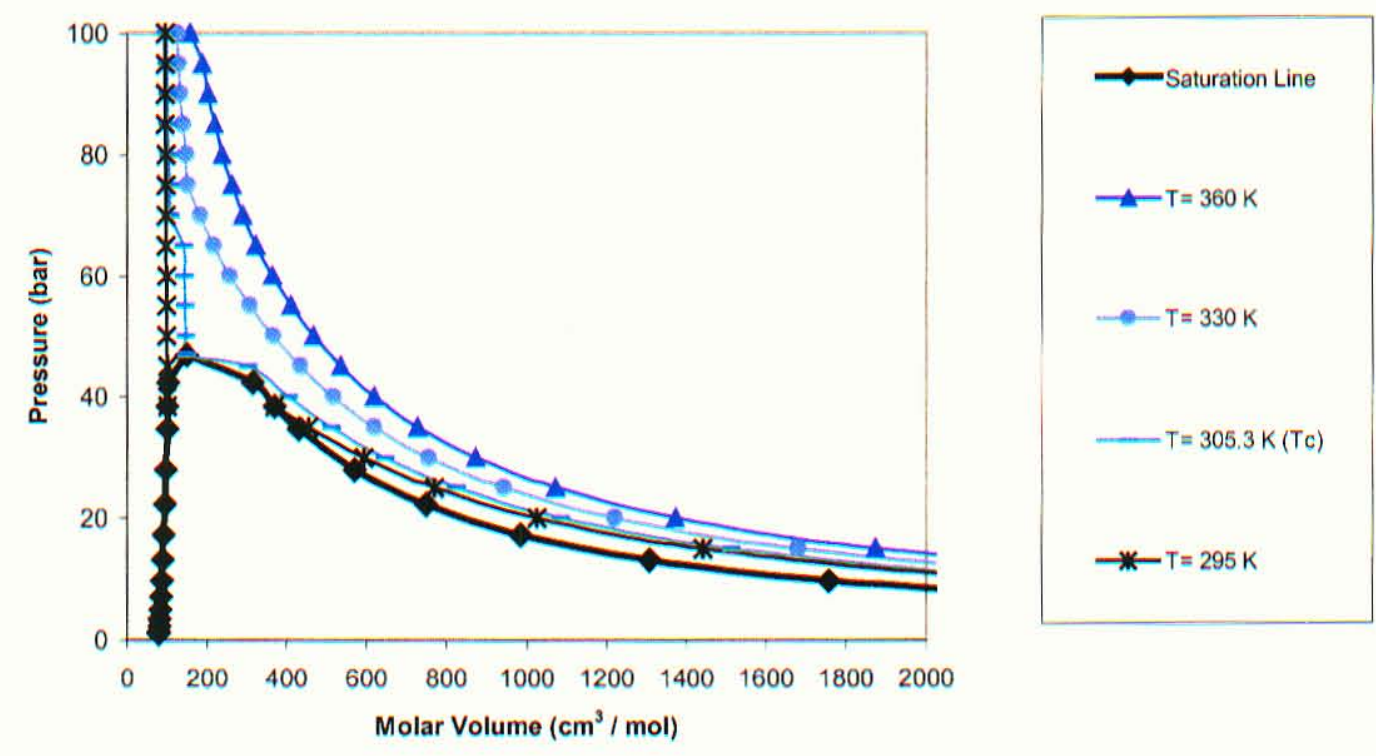

FIGURE 25 - Vapor and Liquid Molar Volumes of Ethane Using the

Modified van der Waals Equation of State 

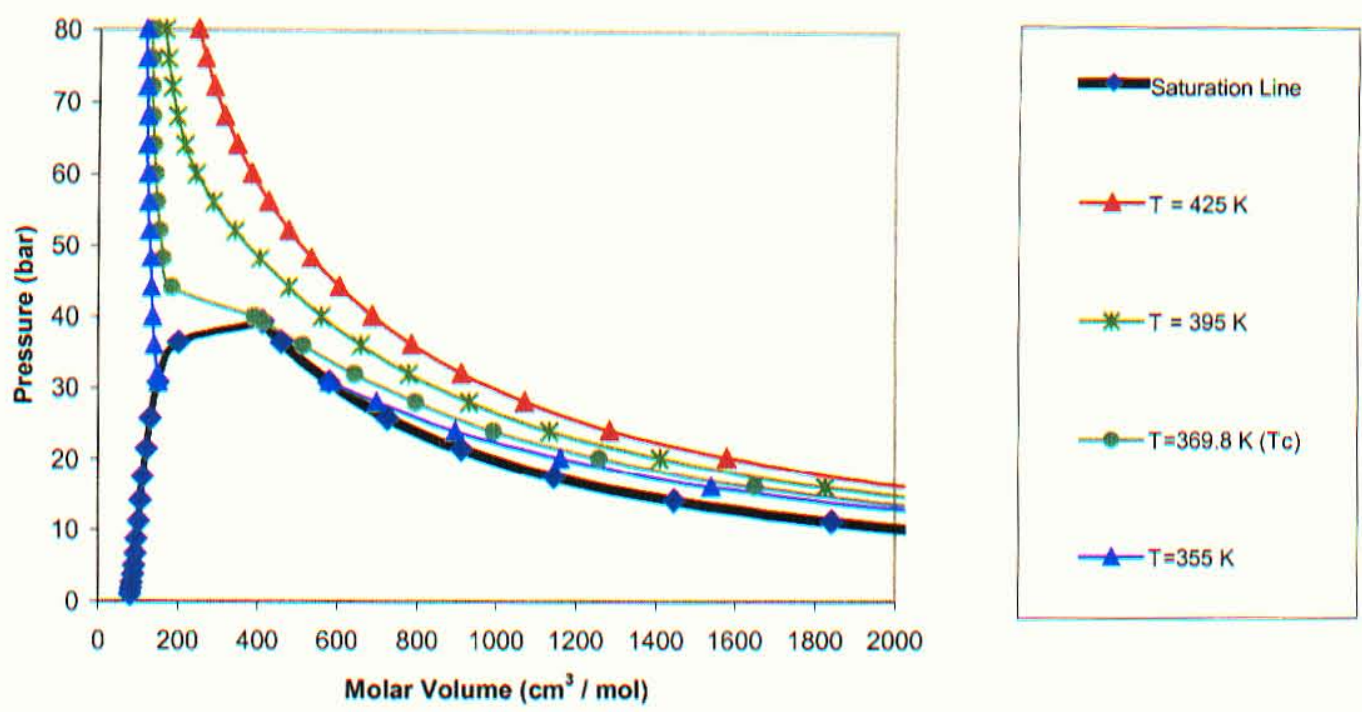

FIGURE 26 - Vapor and Liquid Molar Volumes of Propane Using the

Reidlich-Kwong-Soave Equation of State
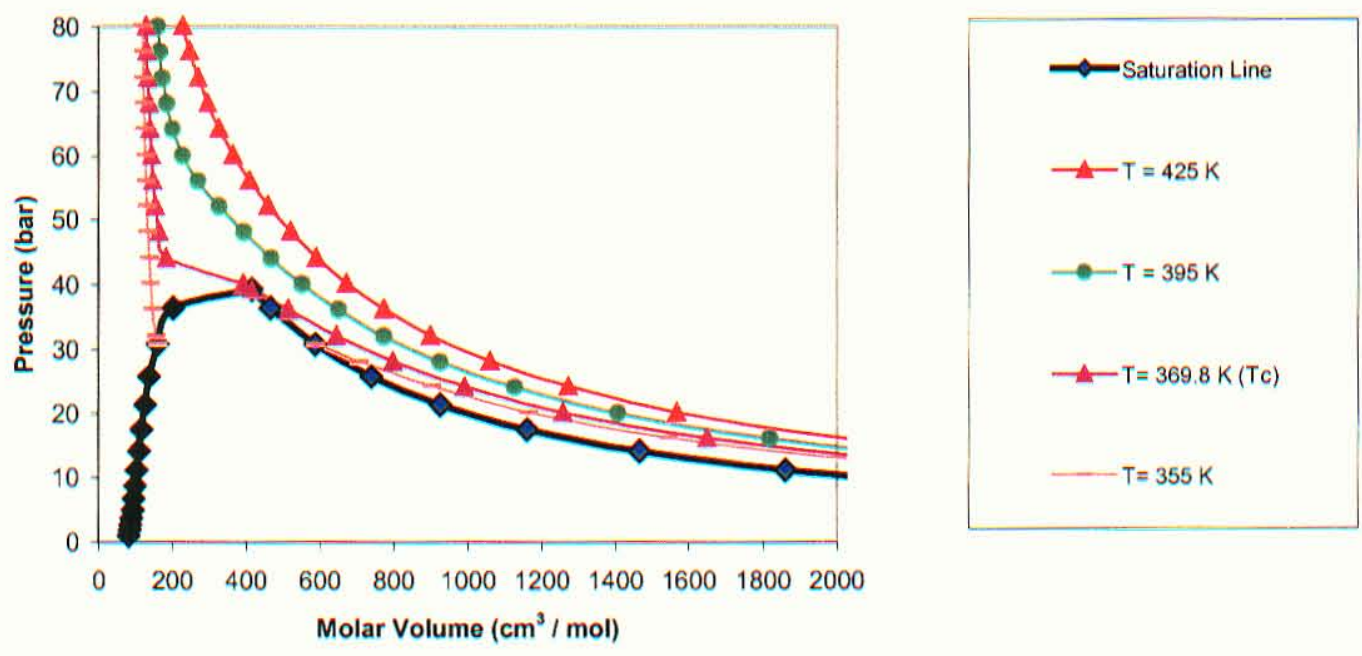

FIGURE 27 - Vapor and Liquid Molar Volumes of Propane Using the Reidlich-Kwong Equation of State 

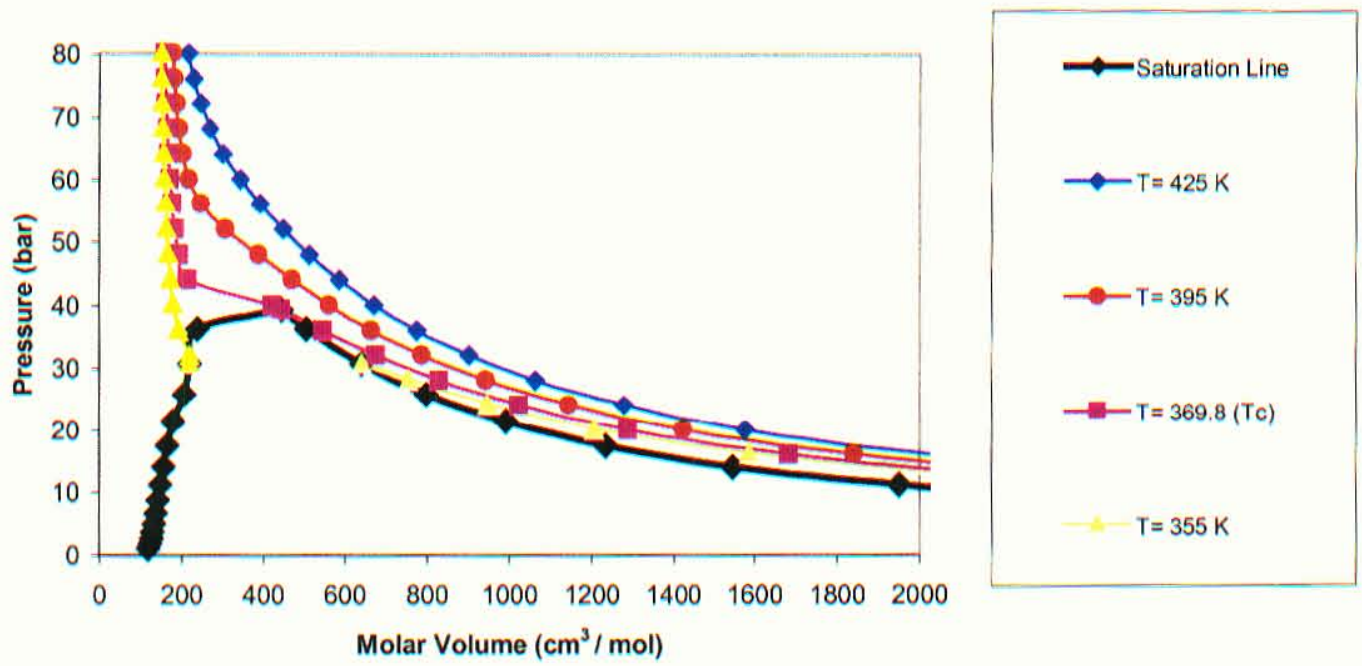

FIGURE 28 - Vapor and Liquid Molar Volumes of Propane Using the

Original van der Waals Equation of State
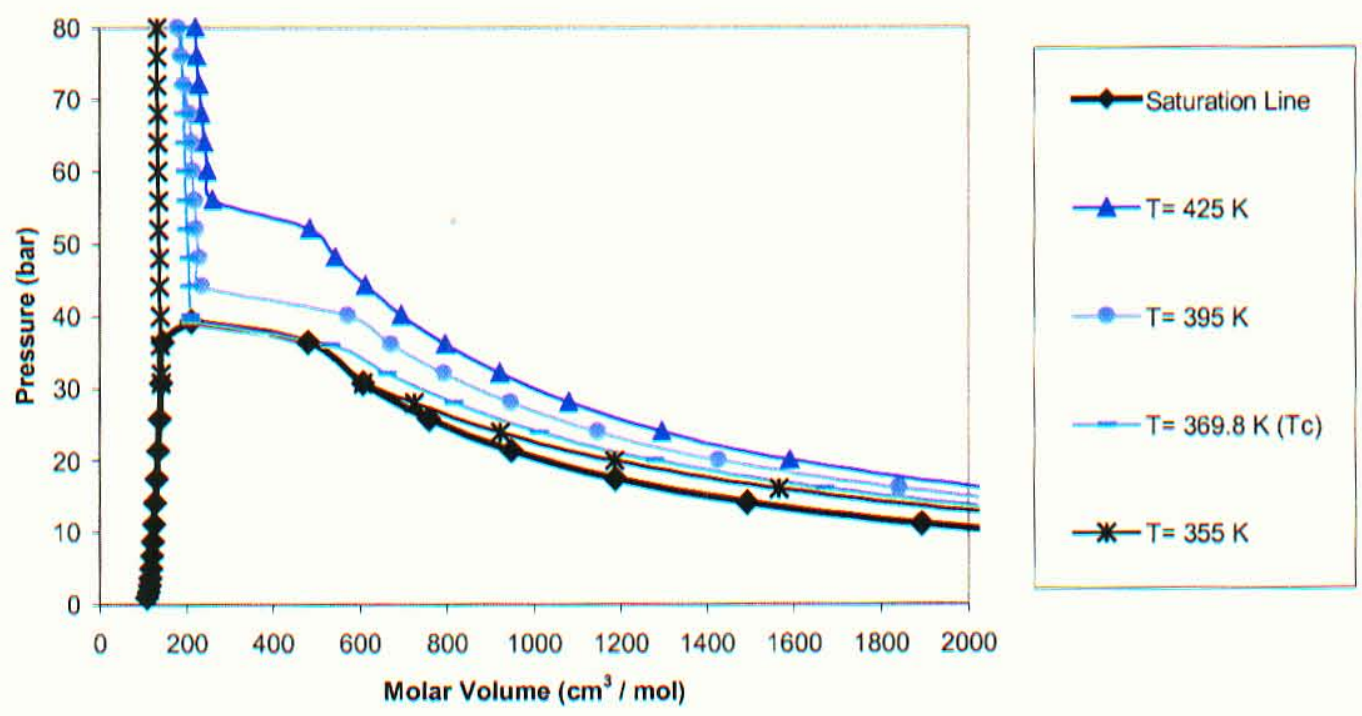

FIGURE 29 - Vapor and Liquid Molar Volumes of Propane Using the Modified van der Waals Equation of State 


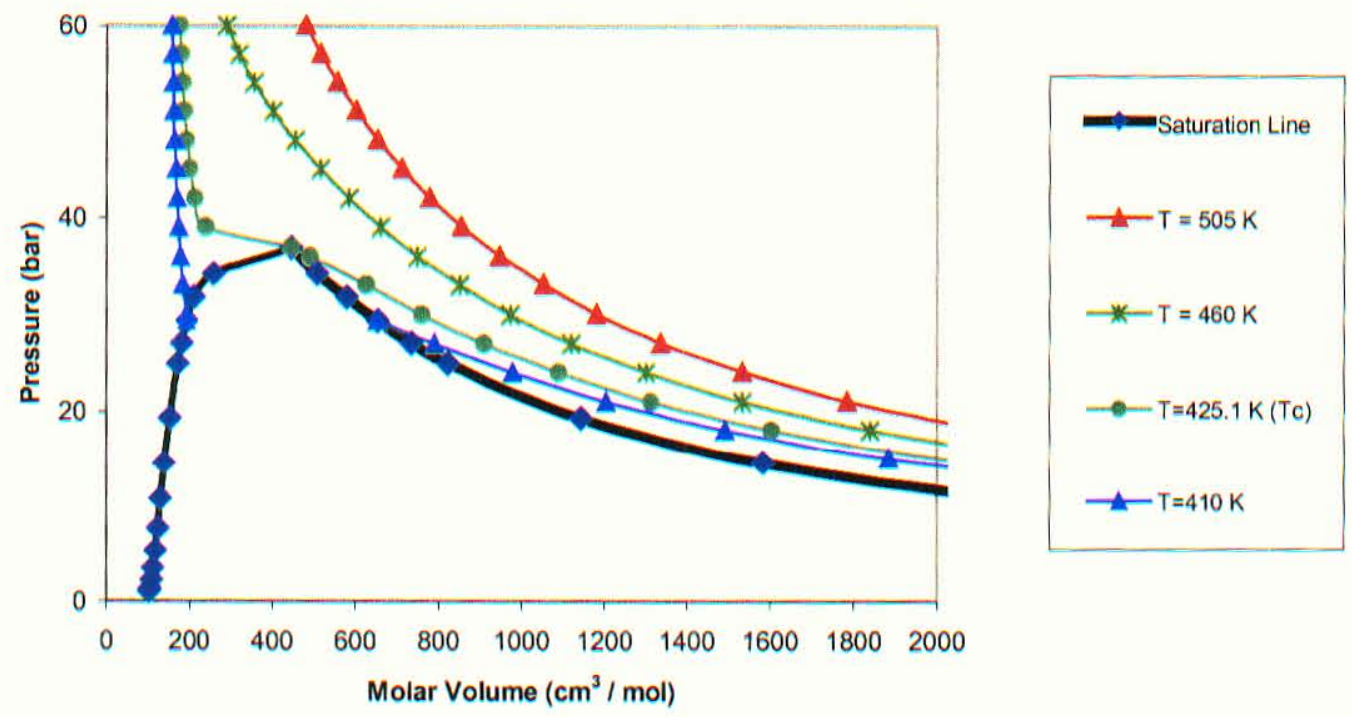

FIGURE 30 - Vapor and Liquid Molar Volumes of $n$-Butane Using the Reidlich-Kwong-Soave Equation of State
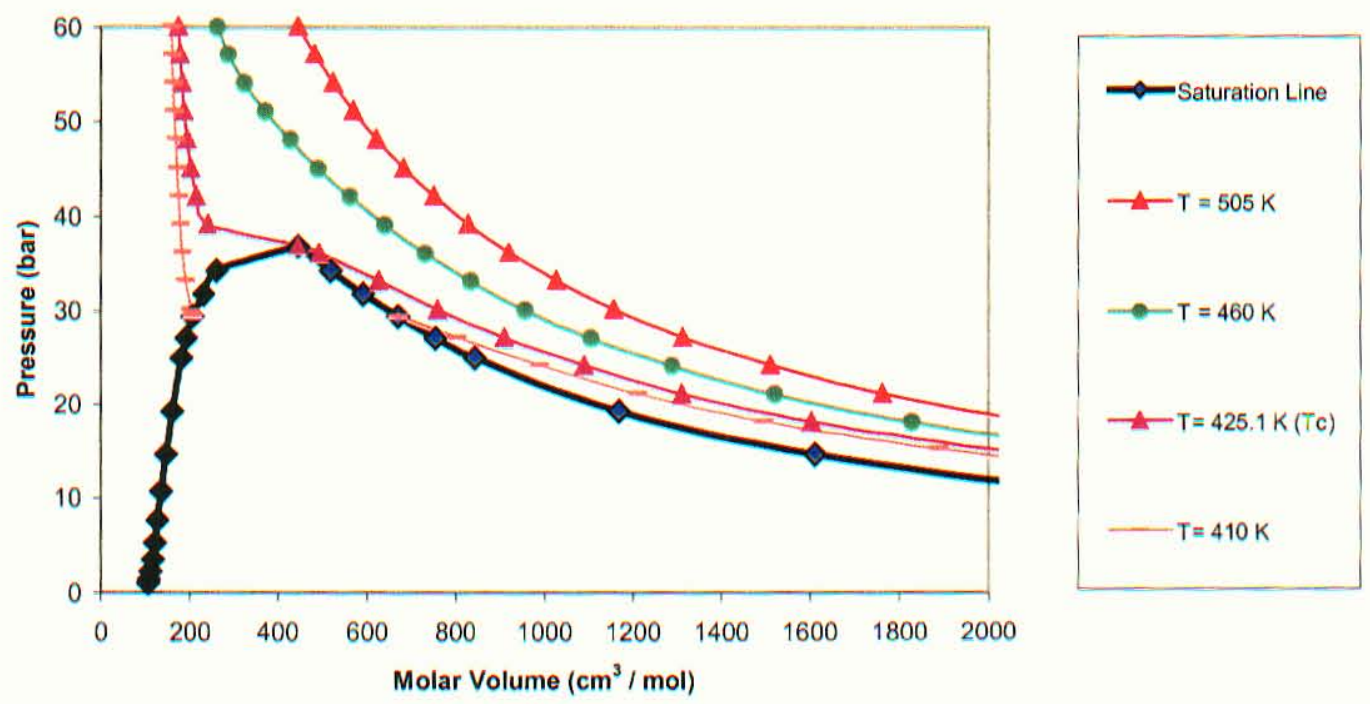

FIGURE 31 - Vapor and Liquid Molar Volumes of $n$-Butane Using the Reidlich-Kwong Equation of State 

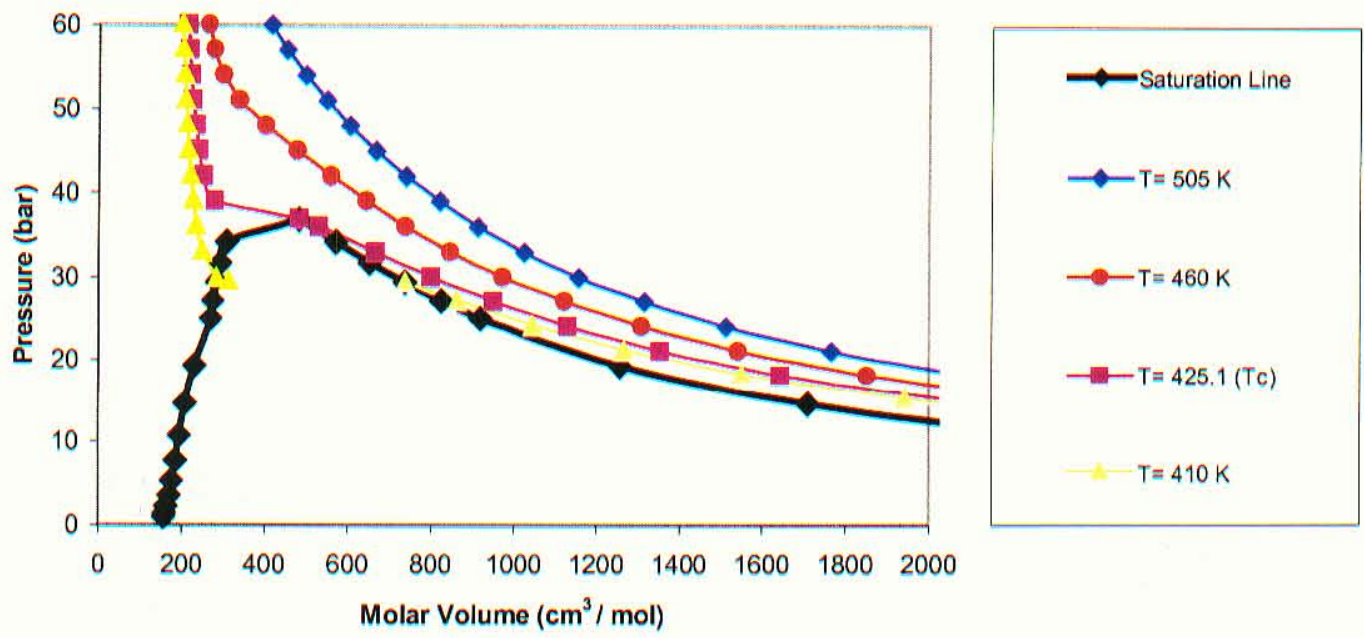

FIGURE 32 - Vapor and Liquid Molar Volumes of $n$-Butane Using the

Original van der Waals Equation of State
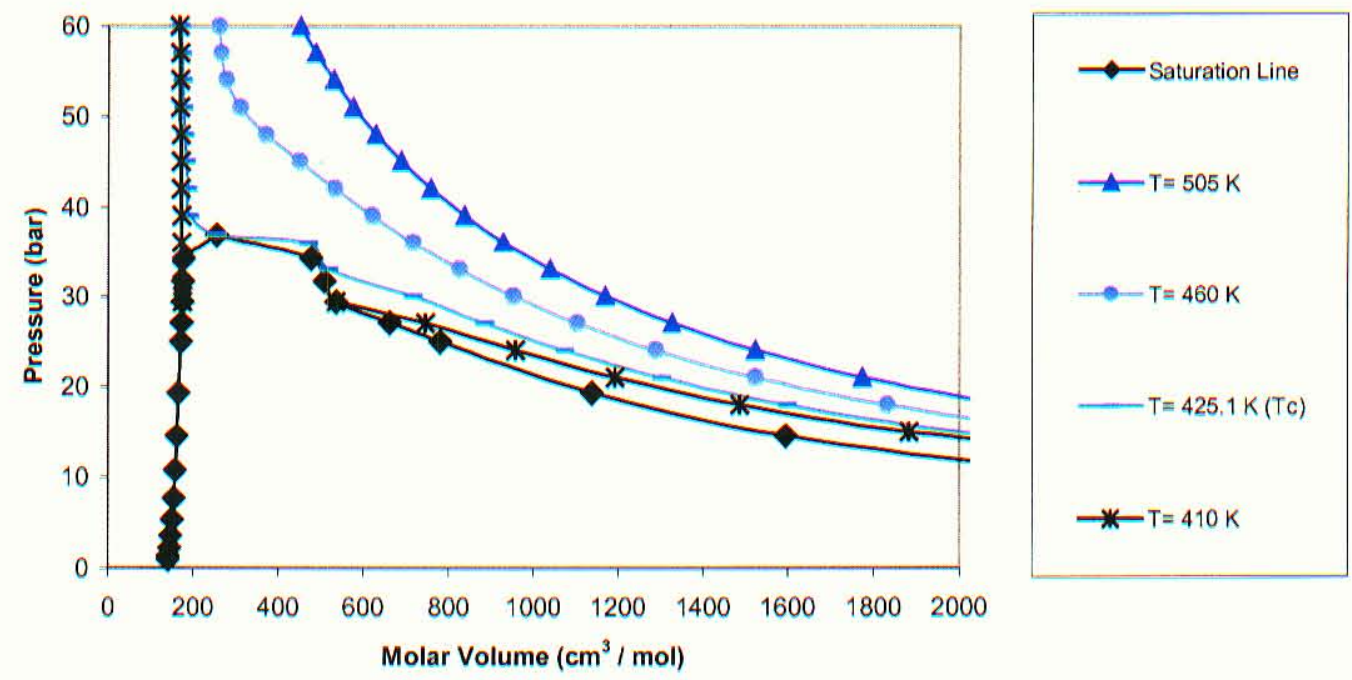

FIGURE 33 - Vapor and Liquid Molar Volumes of $n$-Butane Using the Modified van der Waals Equation of State 


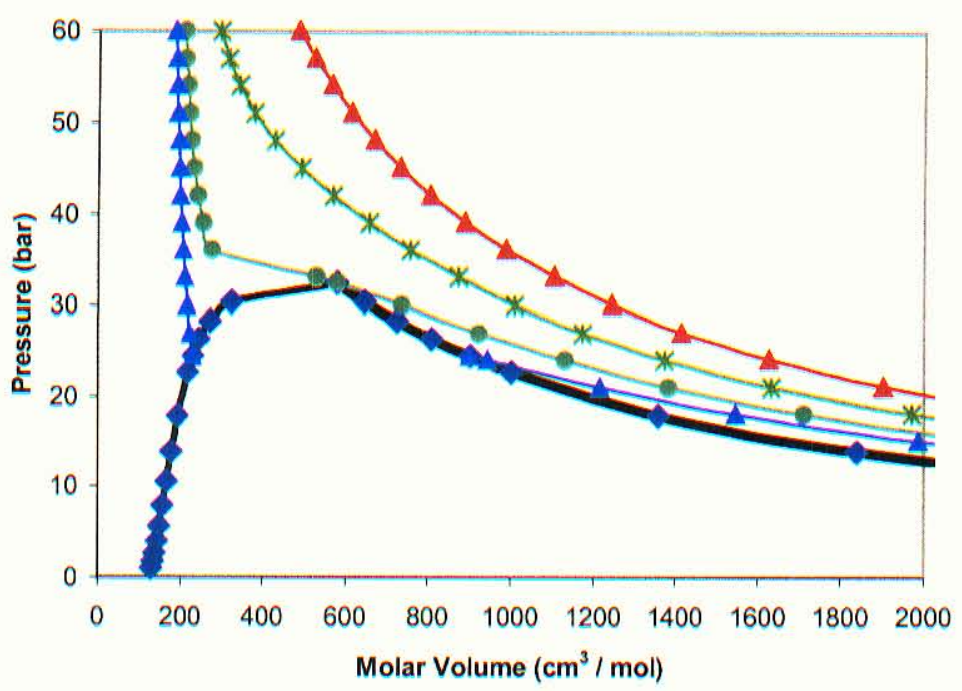

$=$ Saturation Line

$-\mathrm{T}=550 \mathrm{~K}$

$-*-T=505 \mathrm{~K}$

$-\mathrm{T}=469.7 \mathrm{~K}(\mathrm{Tc})$

$-\mathrm{T}=450 \mathrm{~K}$

FIGURE 34 - Vapor and Liquid Molar Volumes of $n$-Pentane Using the

Reidlich-Kwong-Soave Equation of State

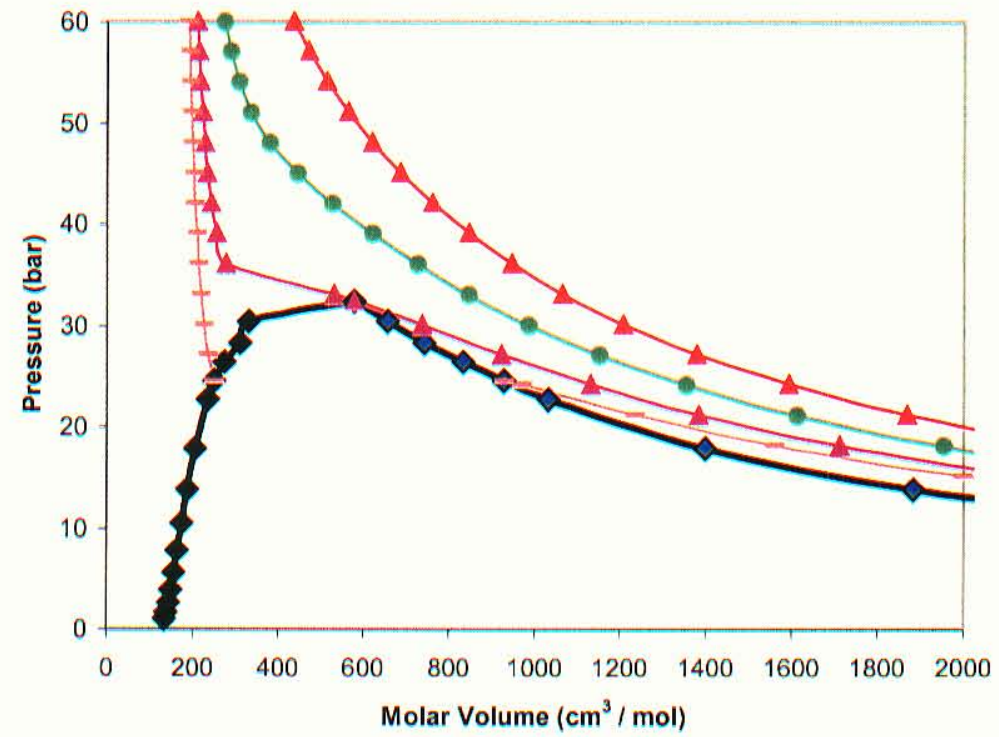

$\approx$ Saturation Line

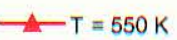

$-\mathrm{T}=505 \mathrm{~K}$

$-\mathrm{T}=469.7 \mathrm{~K}(\mathrm{Tc})$

$-\mathrm{T}=450 \mathrm{~K}$

FIGURE 35 - Vapor and Liquid Molar Volumes of $n$-Pentane Using the

Reidlich-Kwong Equation of State 

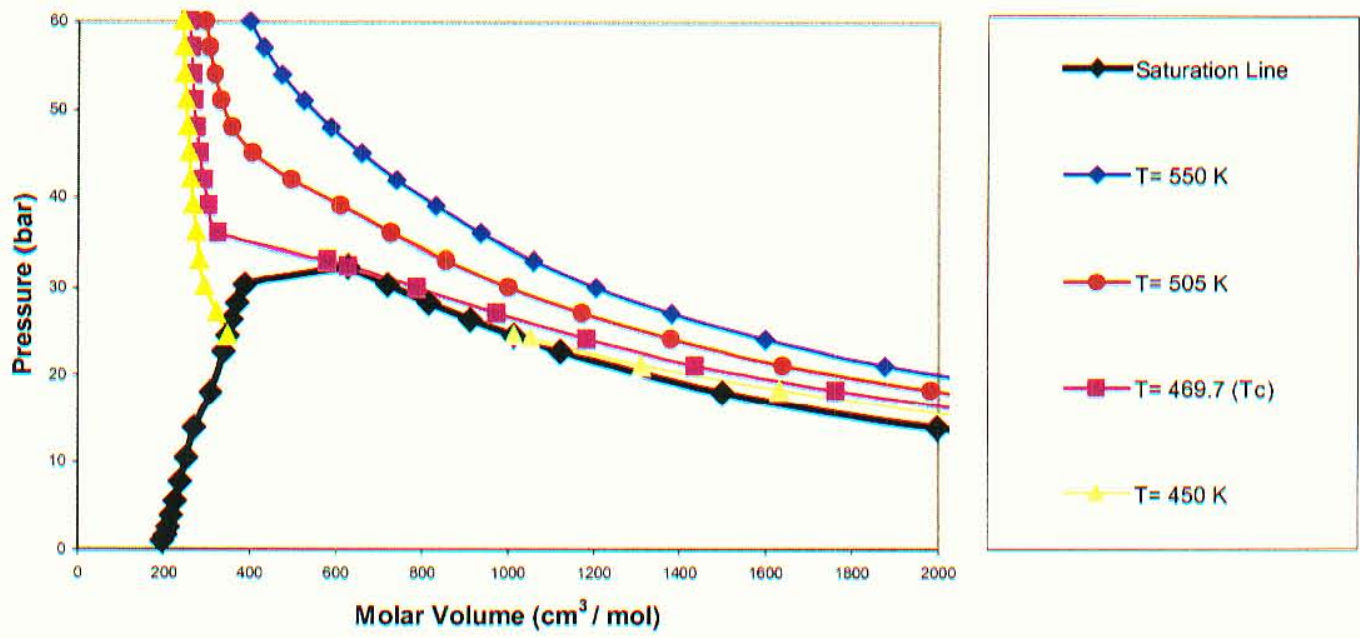

FIGURE 36 - Vapor and Liquid Molar Volumes of $n$-Pentane Using the

Original van der Waals Equation of State
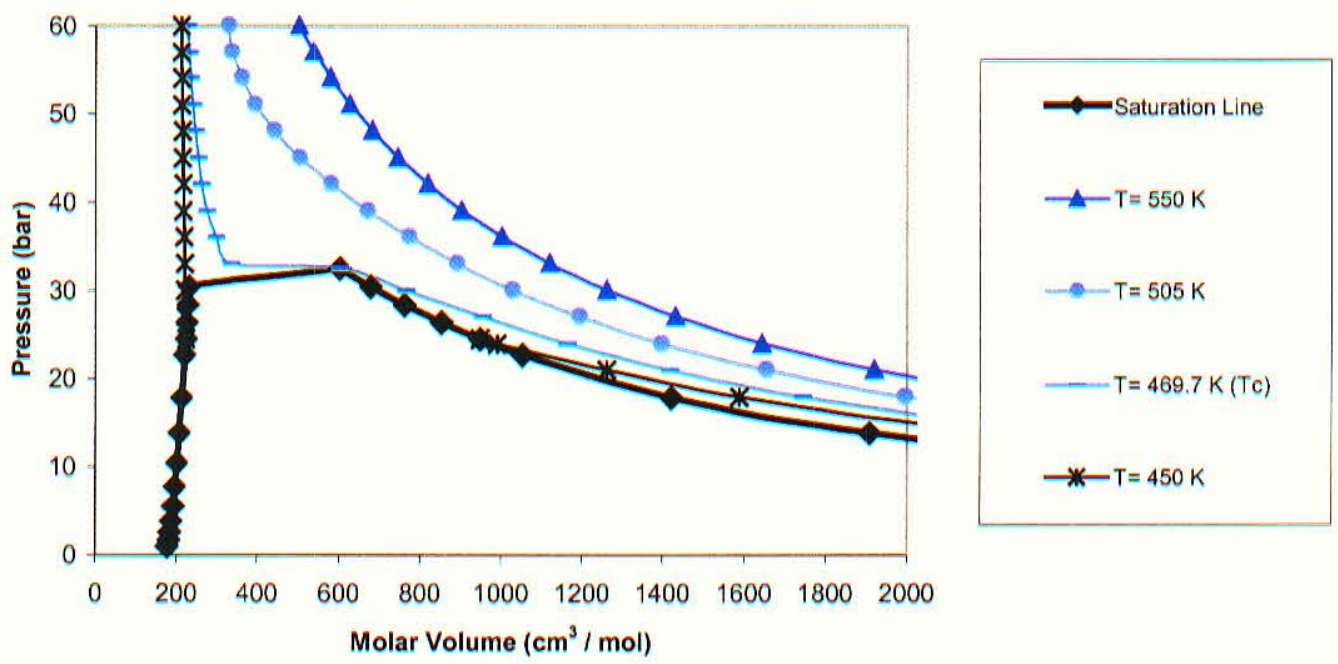

FIGURE 37 - Vapor and Liquid Molar Volumes of $n$-Pentane Using the Modified van der Waals Equation of State 

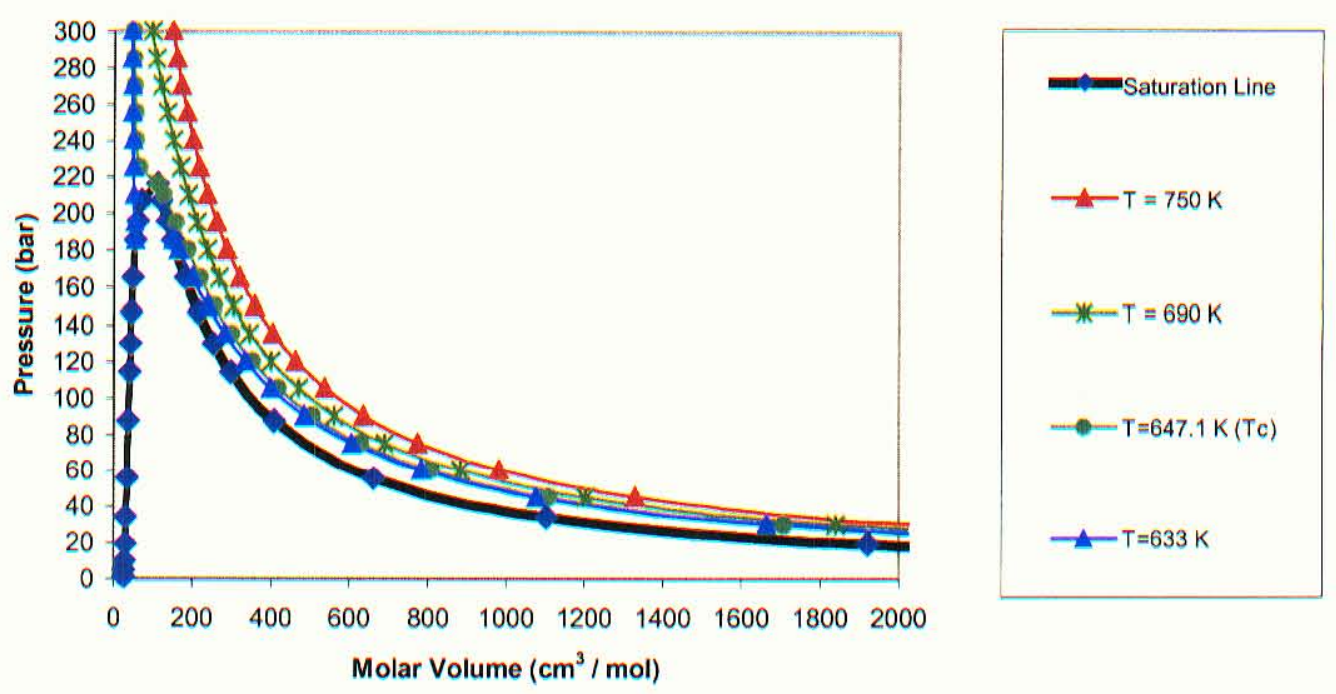

FIGURE 38 - Vapor and Liquid Molar Volumes of Water Using the

Reidlich-Kwong-Soave Equation of State
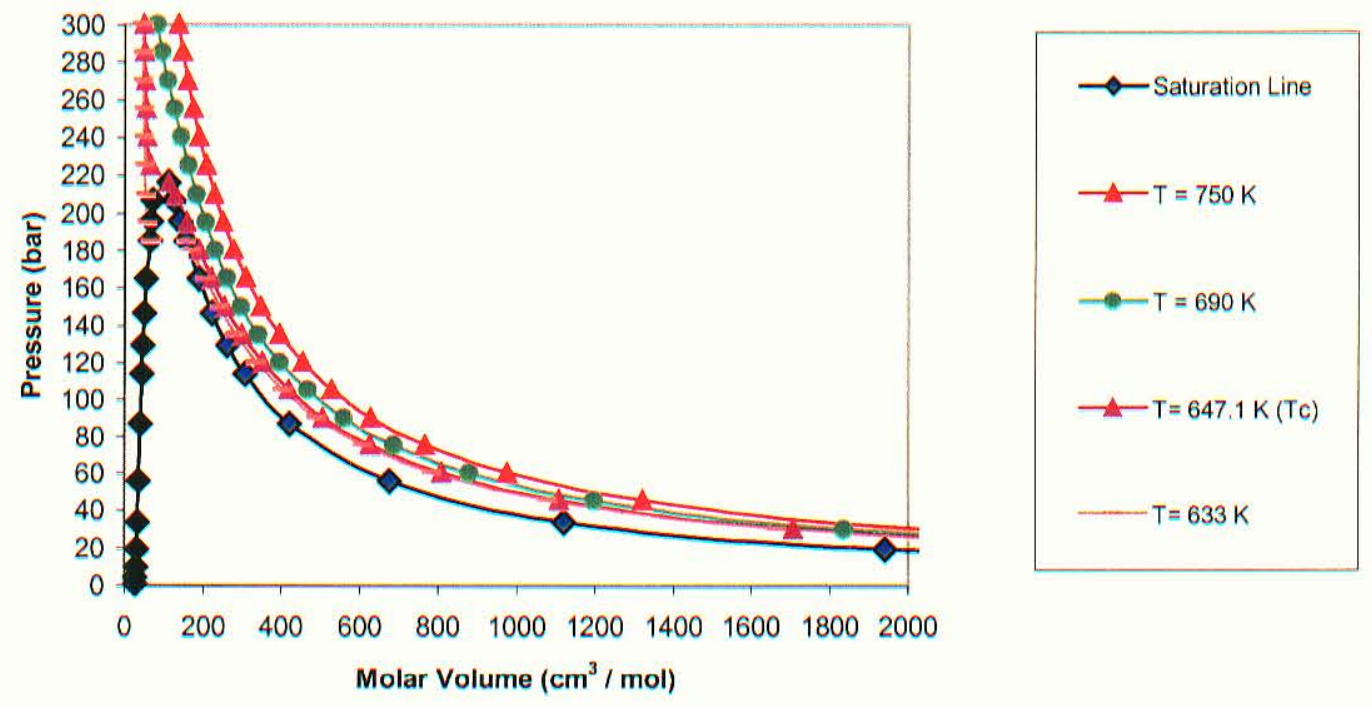

FIGURE 39 - Vapor and Liquid Molar Volumes of Water Using the Reidlich-Kwong Equation of State 

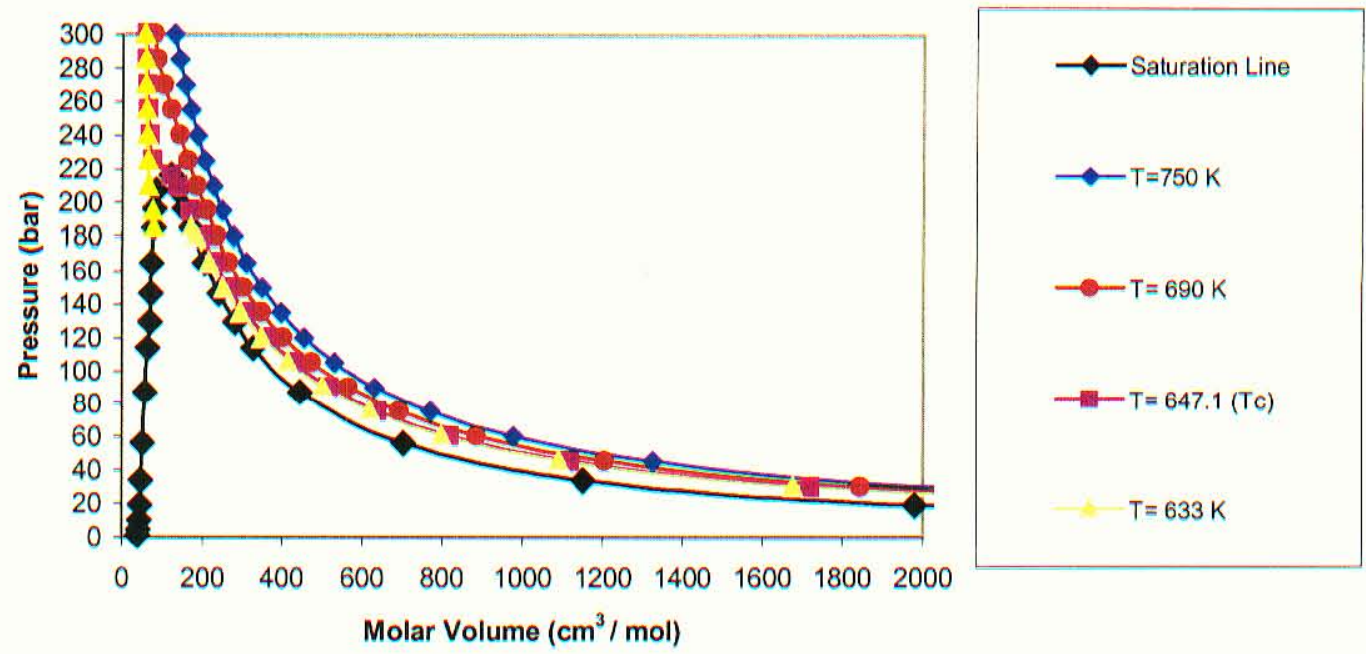

FIGURE 40 - Vapor and Liquid Molar Volumes of Water Using the

Original van der Waals Equation of State
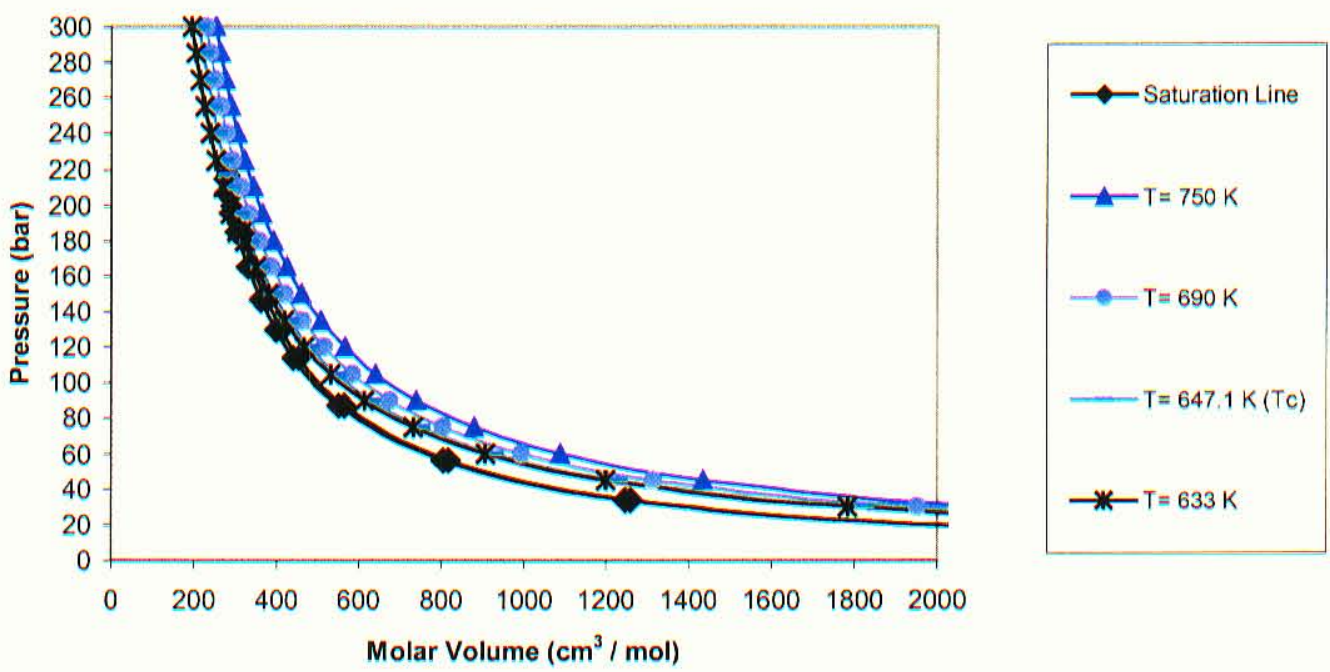

FIGURE 41 - Vapor and Liquid Molar Volumes of Water Using the

Modified van der Waals Equation of State 
APPENDIX II

PRESSURE-MOLAR ENTHALPY GRAPHS 

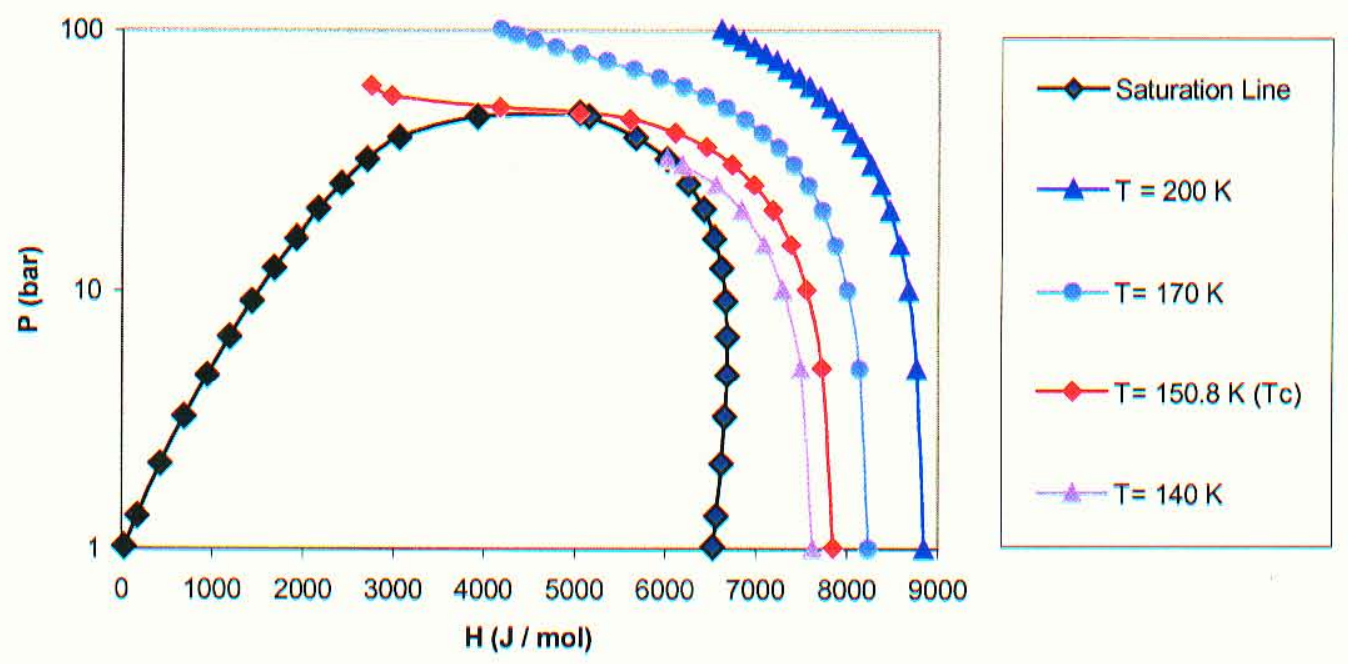

FIGURE 6 - Vapor and Liquid Molar Enthalpies of Argon Using the

Reidlich-Kwong Equation of State
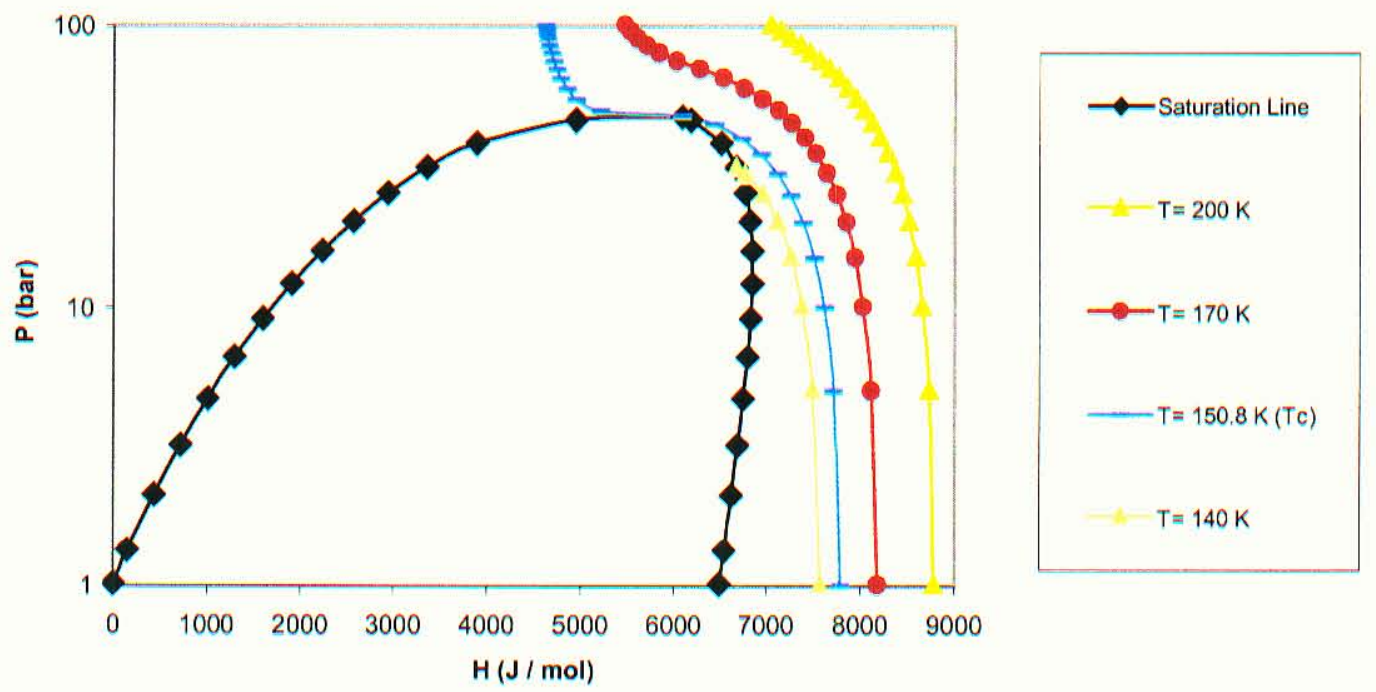

FIGURE 42 - Vapor and Liquid Molar Enthalpies of Argon Using the

Original van der Waals Equation of State 


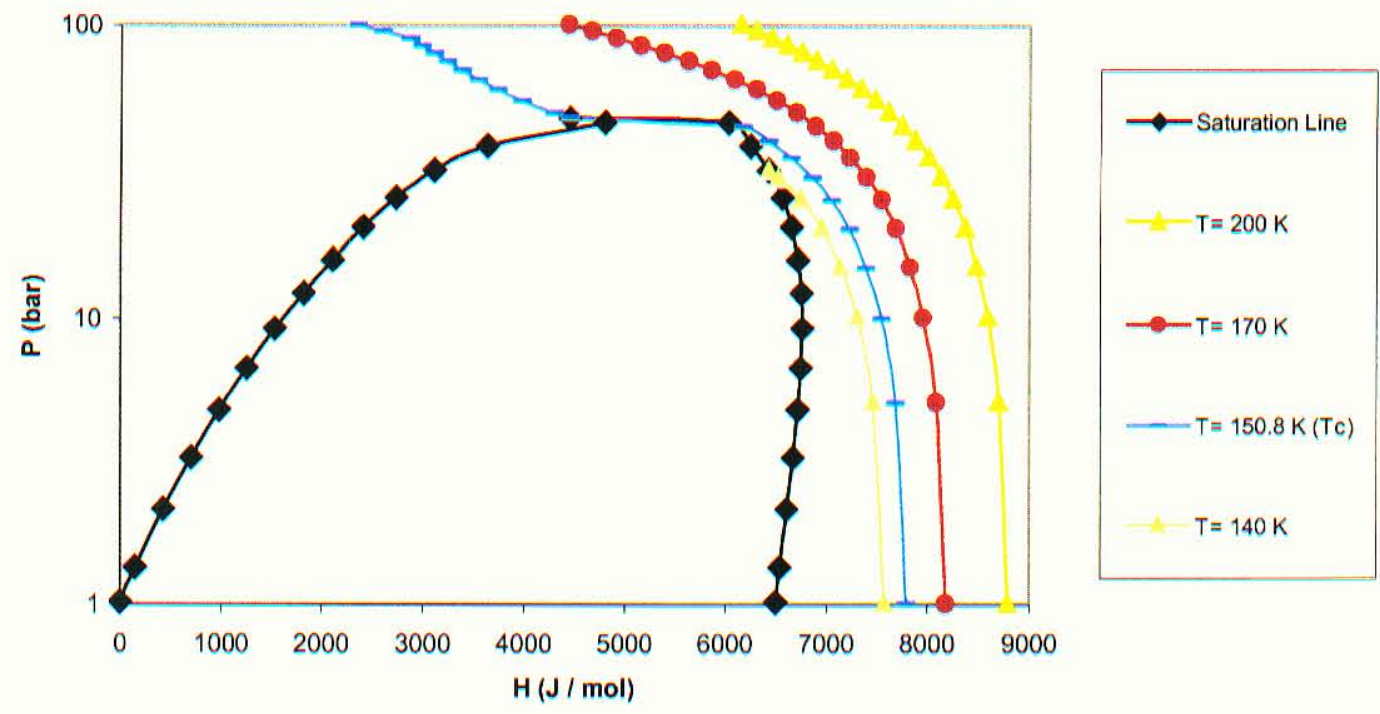

FIGURE 43 - Vapor and Liquid Molar Enthalpies of Argon Using the Modified van der Waals Equation of State

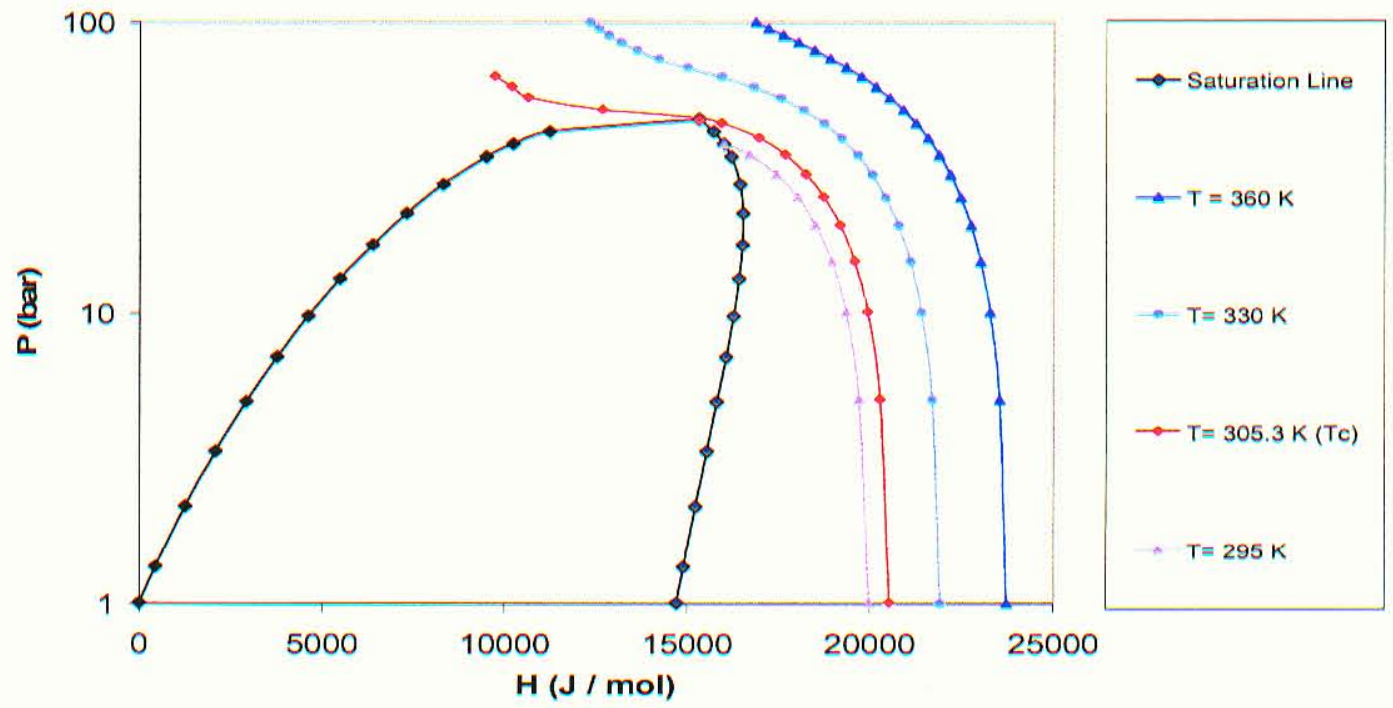

FIGURE 44 - Vapor and Liquid Molar Enthalpies of Ethane Using the Reidlich-Kwong Equation of State 

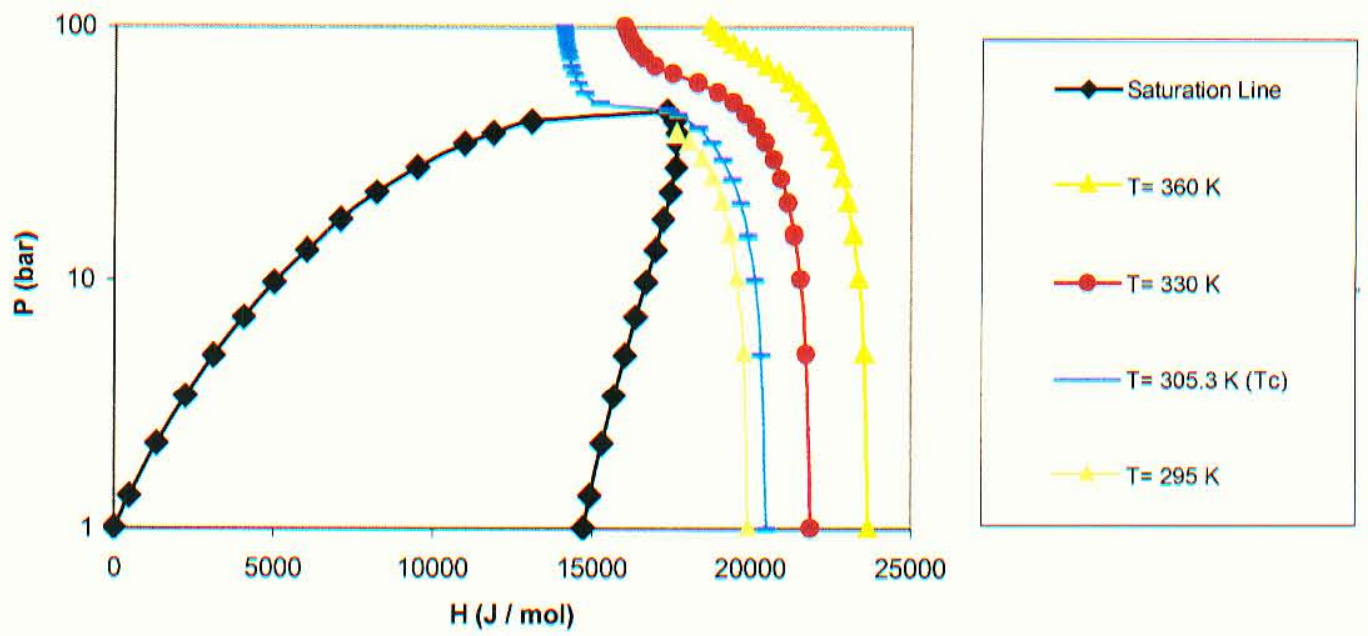

FIGURE 45 - Vapor and Liquid Molar Enthalpies of Ethane Using the Original van der Waals Equation of State
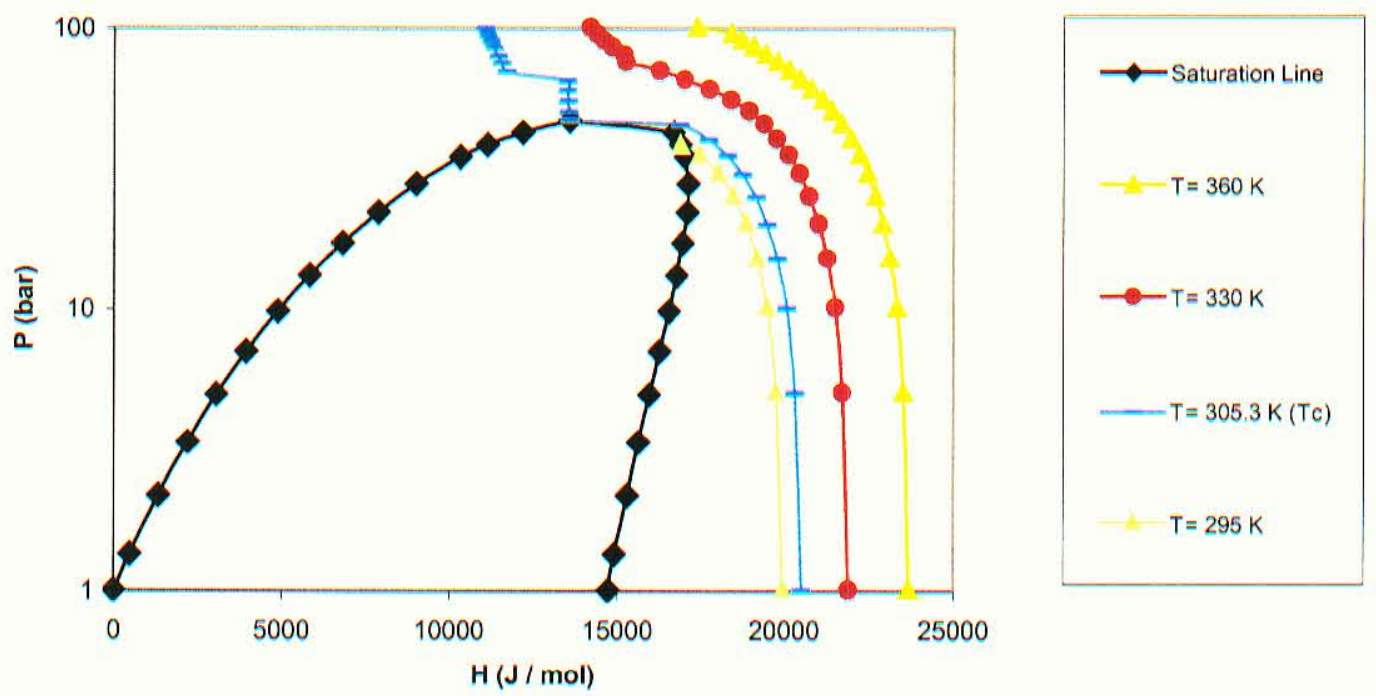

FIGURE 46 - Vapor and Liquid Molar Enthalpies of Ethane Using the Modified van der Waals Equation of State 


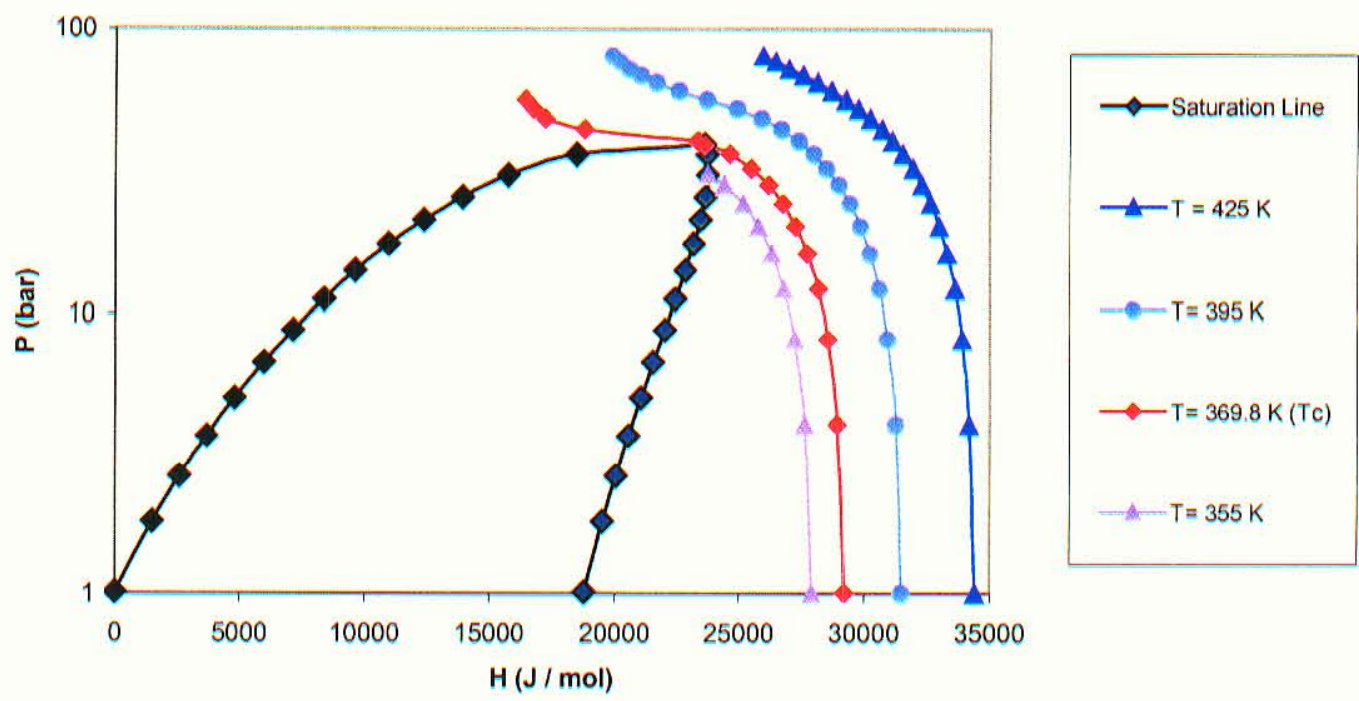

FIGURE 47 - Vapor and Liquid Molar Enthalpies of Propane Using the Reidlich-Kwong Equation of State
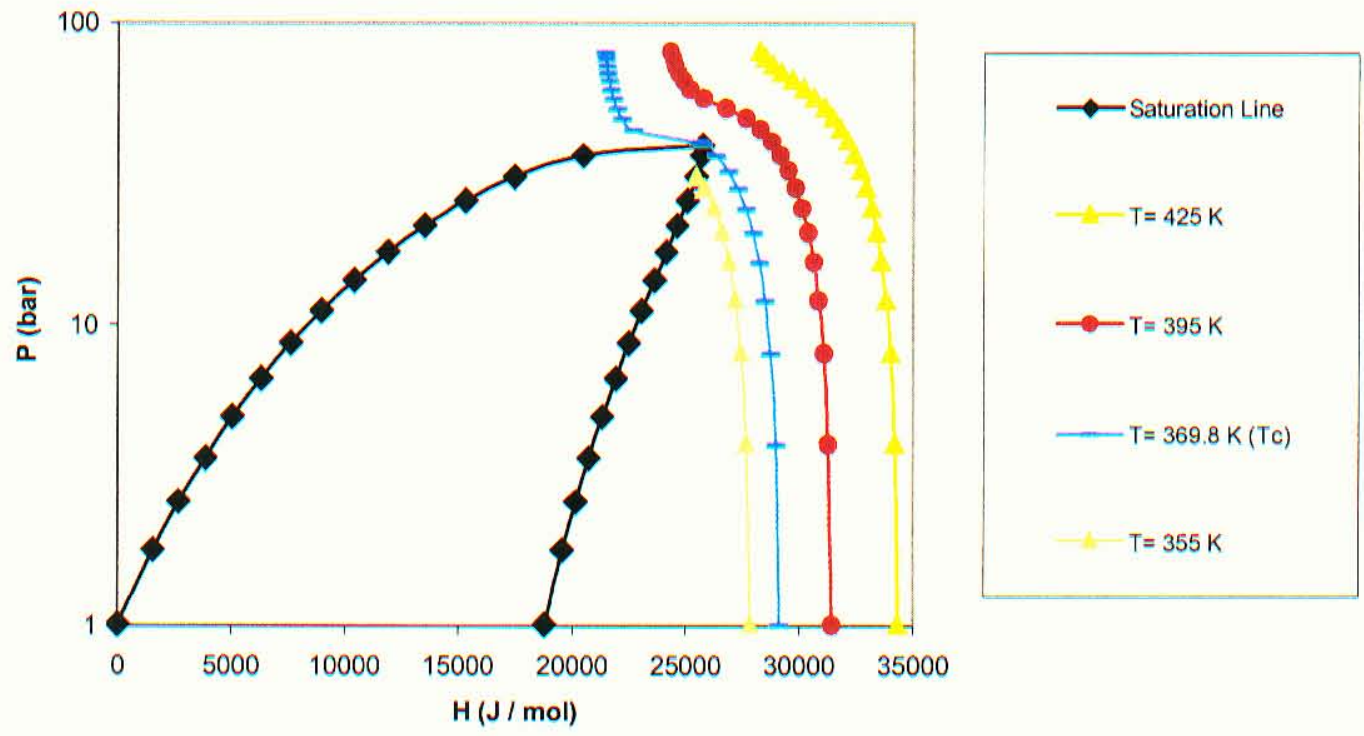

FIGURE 48 - Vapor and Liquid Molar Enthalpies of Propane Using the Original van der Waals Equation of State 

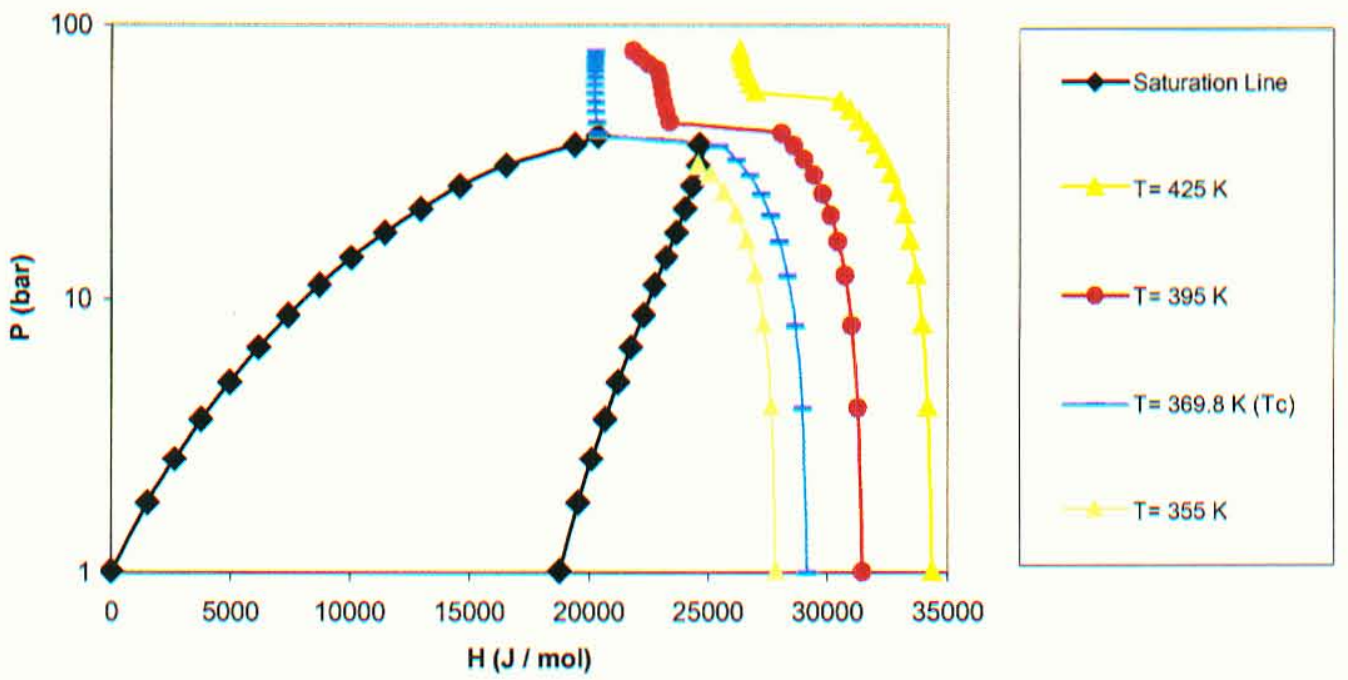

FIGURE 49 - Vapor and Liquid Molar Enthalpies of Propane Using the

Modified van der Waals Equation of State
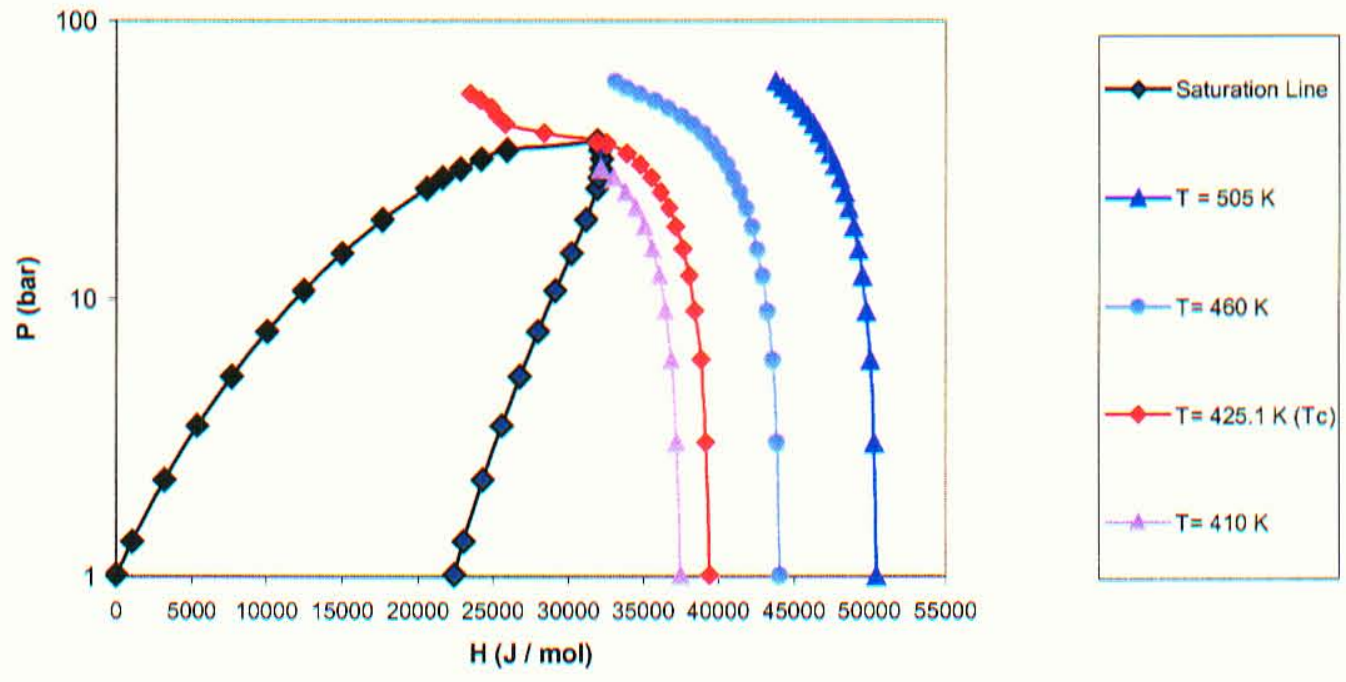

FIGURE 50 - Vapor and Liquid Molar Enthalpies of $n$-Butane Using the

Reidlich-Kwong Equation of State 


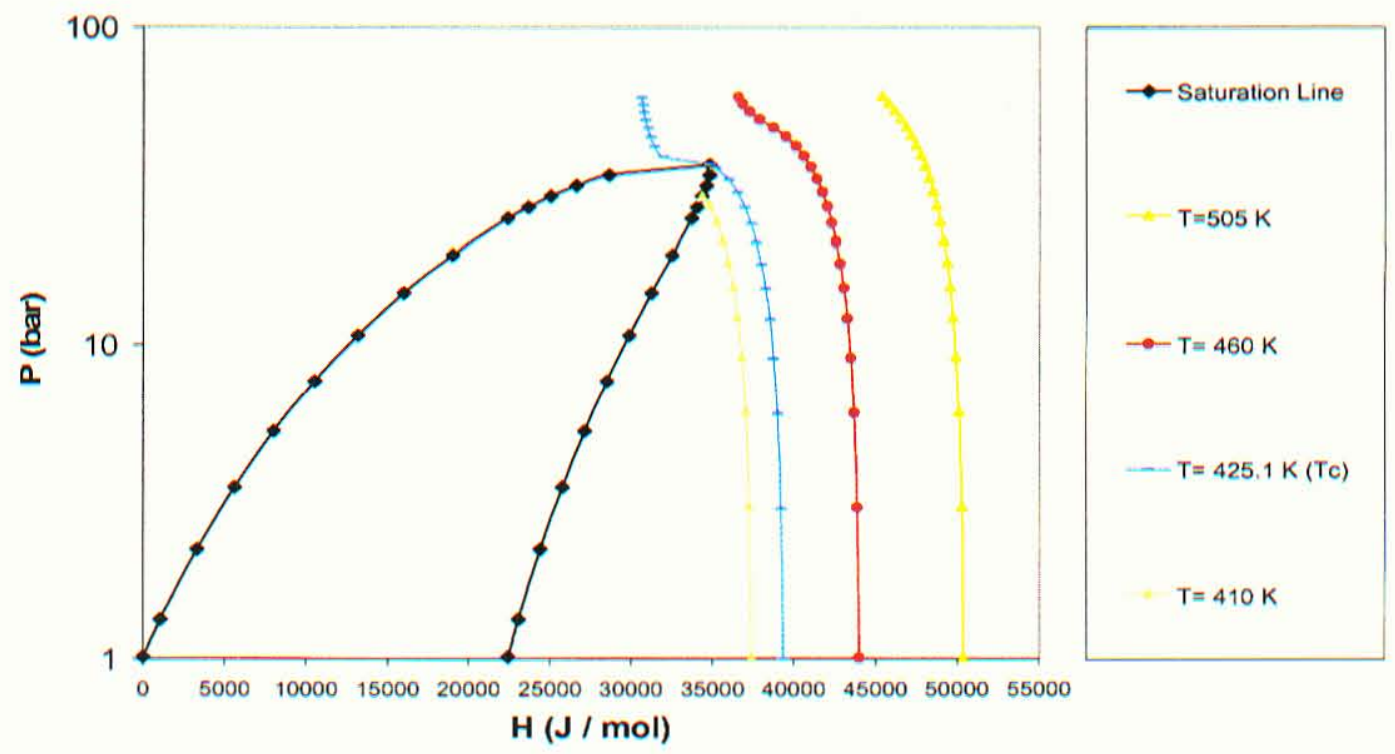

FIGURE 51 - Vapor and Liquid Molar Enthalpies of $n$-Butane Using the Original van der Waals Equation of State
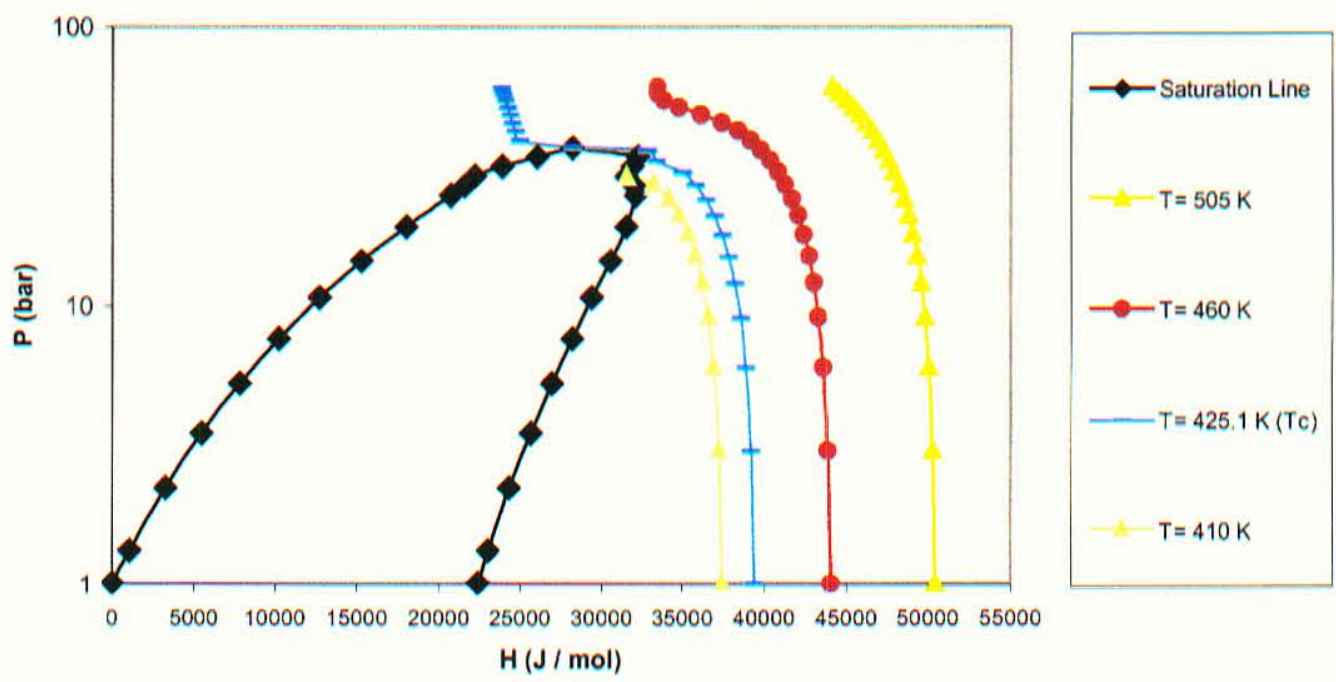

FIGURE 52 - Vapor and Liquid Molar Enthalpies of $n$-Butane Using the Modified van der Waals Equation of State 


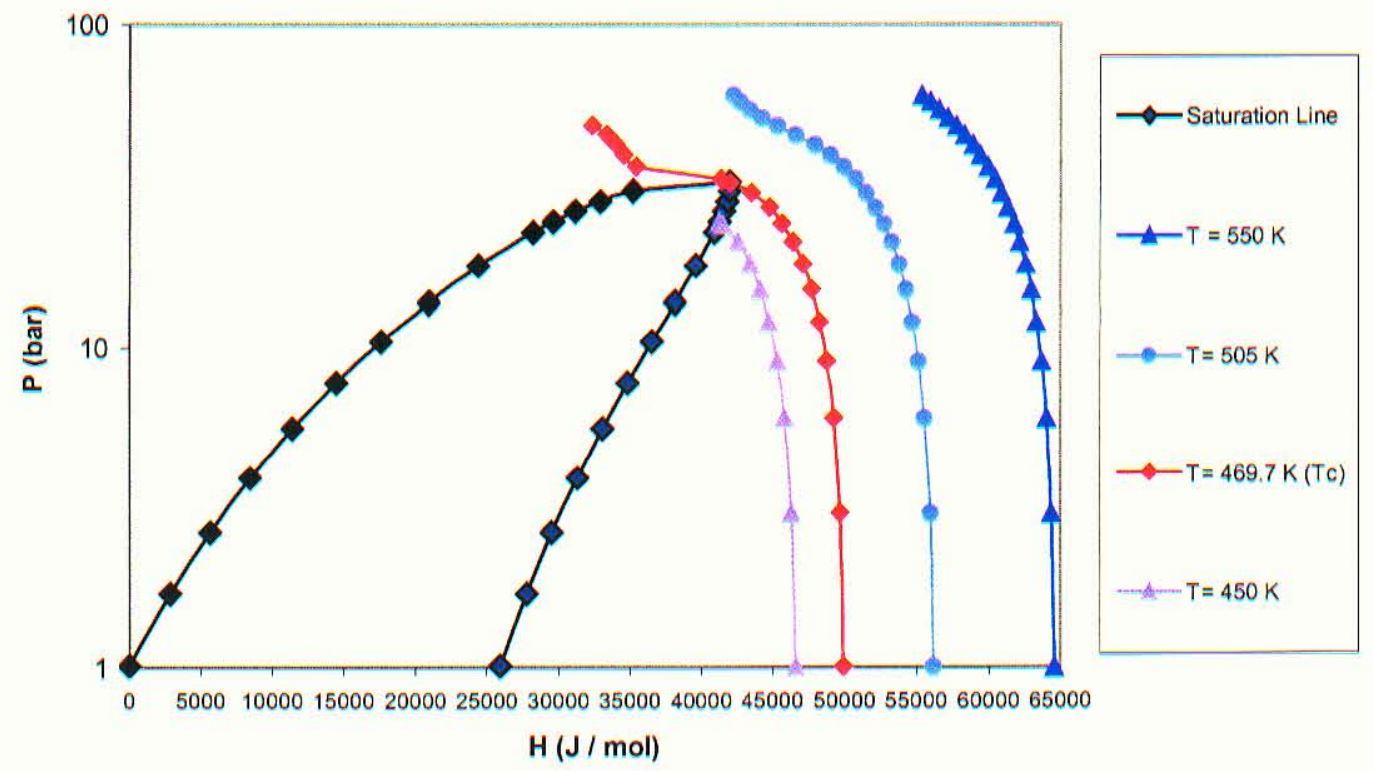

FIGURE 53 - Vapor and Liquid Molar Enthalpies of $n$-Pentane Using the Reidlich-Kwong Equation of State
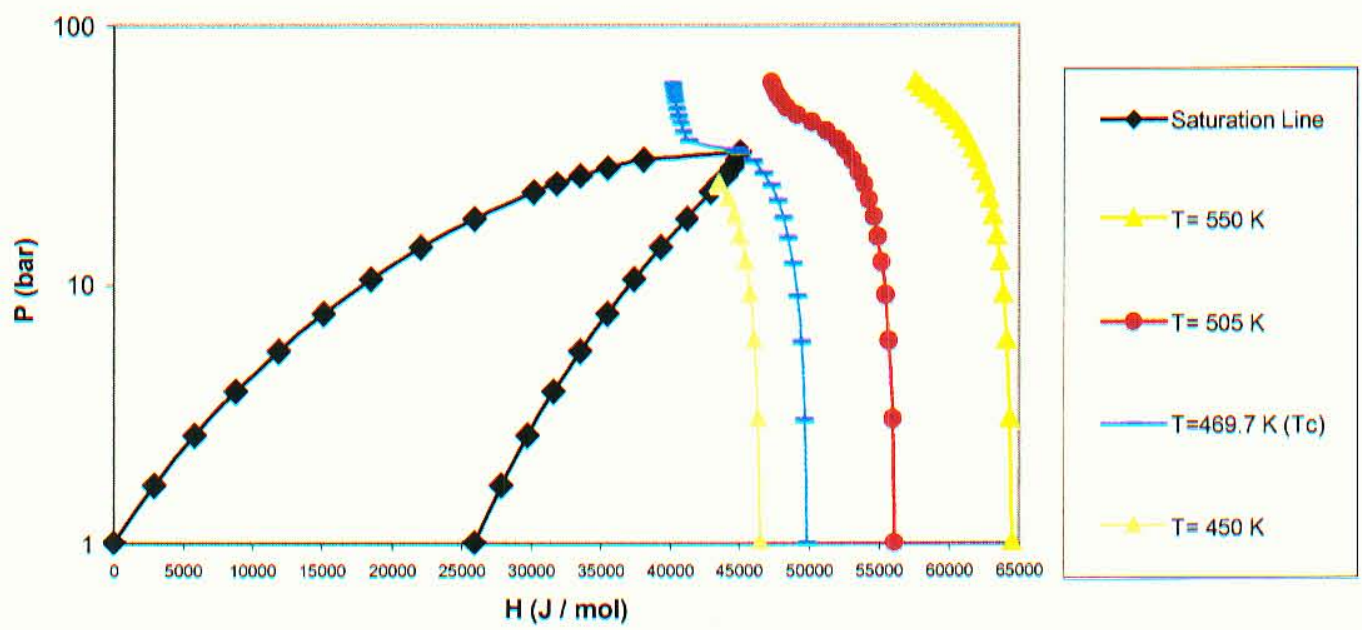

FIGURE 54 - Vapor and Liquid Molar Enthalpies of $n$-Pentane Using the

Original van der Waals Equation of State 


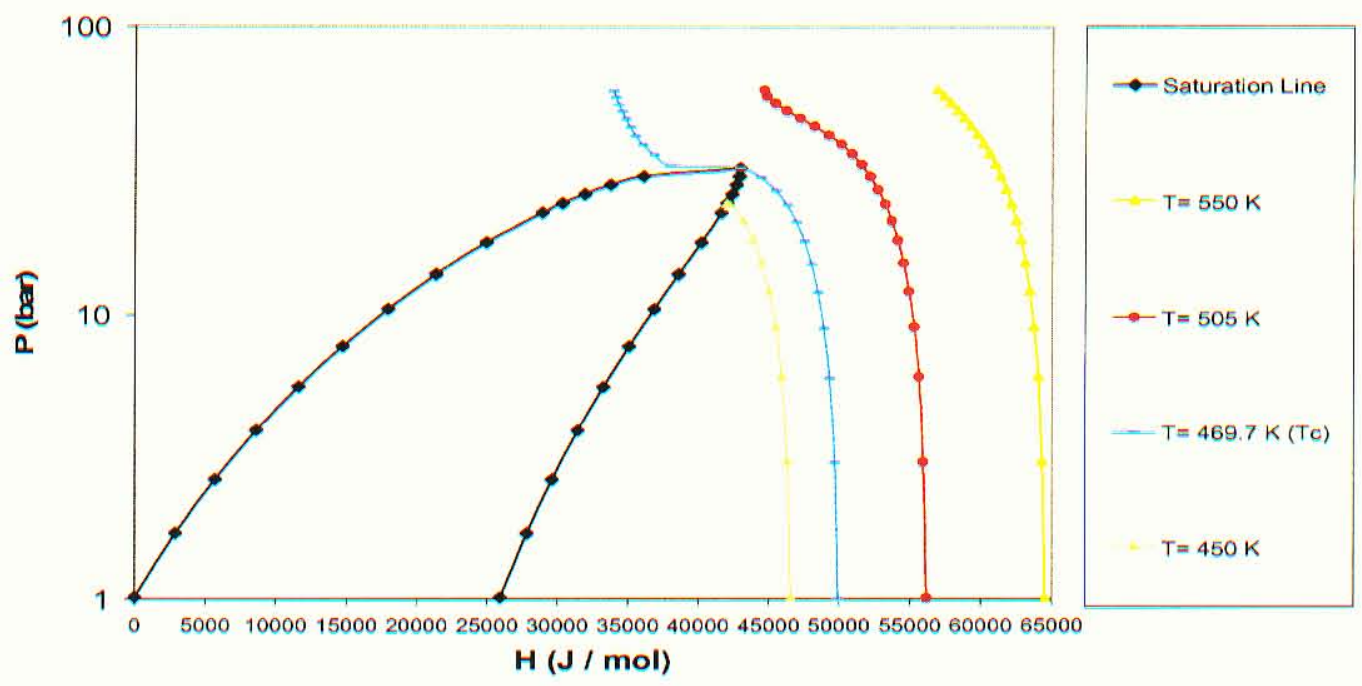

FIGURE 55 - Vapor and Liquid Molar Enthalpies of $n$-Pentane Using the Modified van der Waals Equation of State

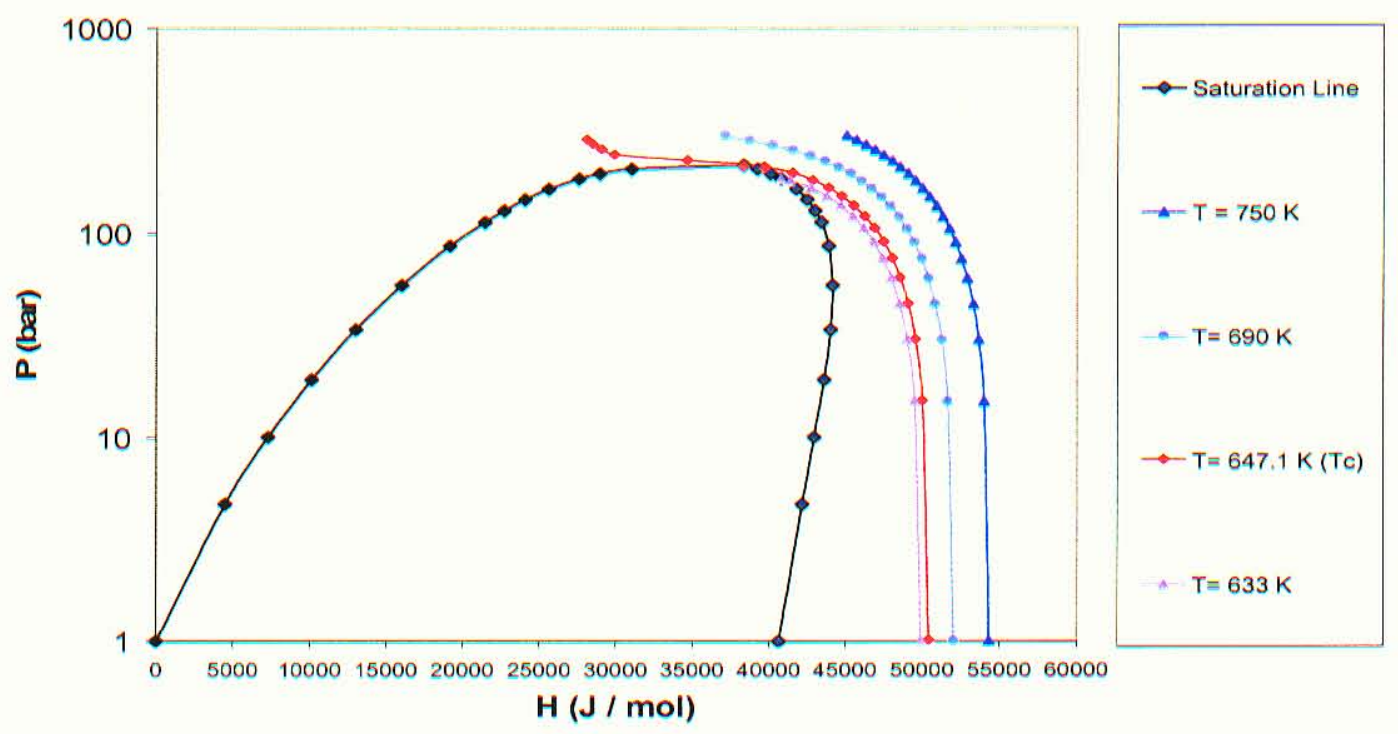

FIGURE 56 - Vapor and Liquid Molar Enthalpies of Water Using the

Reidlich-Kwong Equation of State 


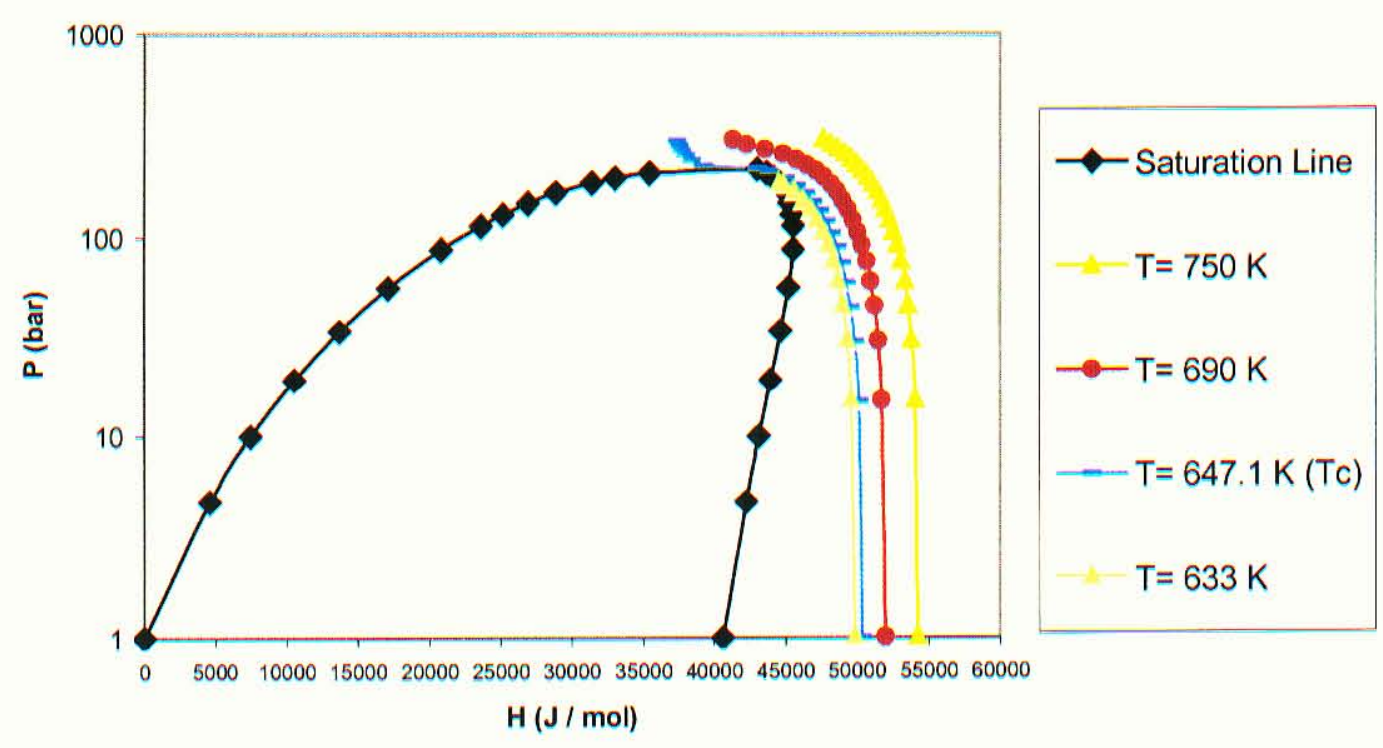

FIGURE 57 - Vapor and Liquid Molar Enthalpies of Water Using the

Original van der Waals Equation of State
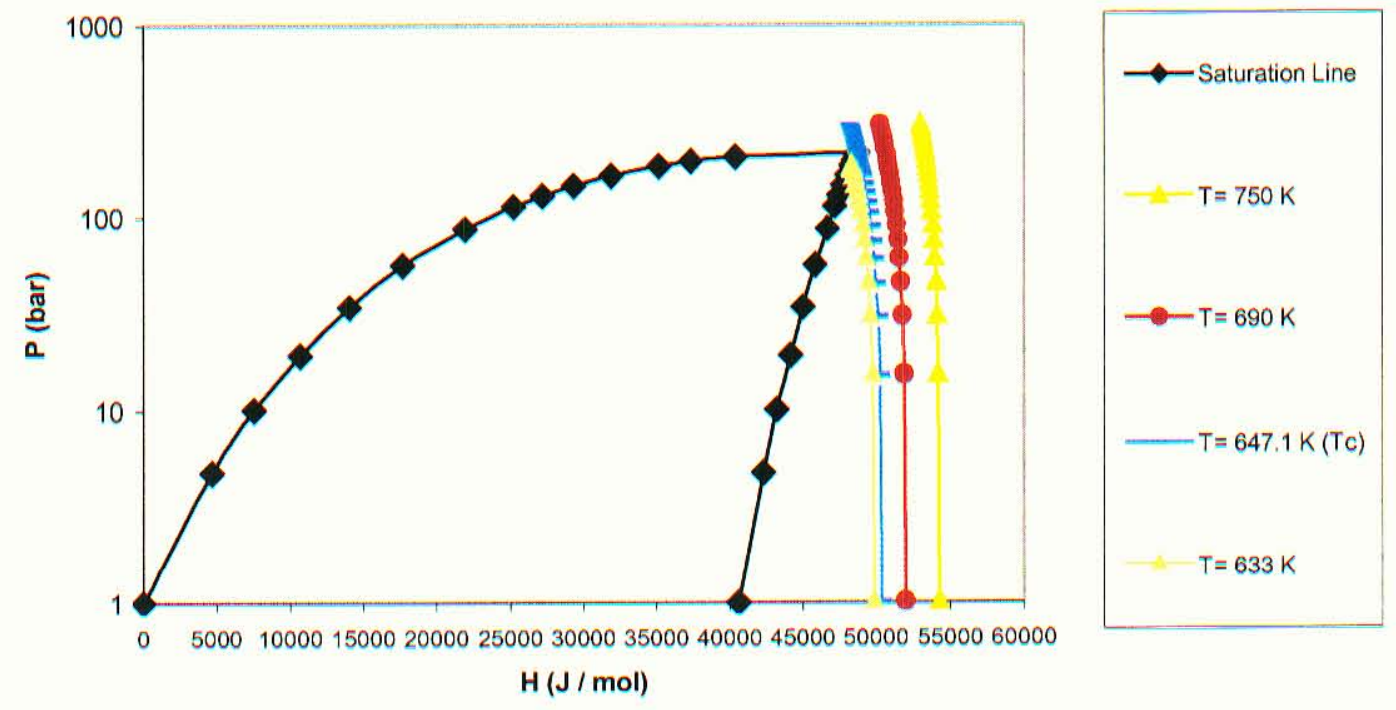

FIGURE 58 - Vapor and Liquid Molar Enthalpies of Water Using the Modified van der Waals Equation of State 


\section{APPENDIX III}

RESIDUAL ENTHALPY CALCULATIONS 


\section{A. Reidlich-Kwong Equation of State}

Equation of State: $P=R T /(v-b)-a / T^{1 / 2} * v *(v+b)$

$\mathrm{Z}=$ Compressibility $=P v / R T=v / R T *\left[R T /(v-b)-a / T^{1 / 2} * v *(v+b)\right]$

$Z=v /(v-b)-a / T^{1 / 2} *(v+b) R T$

$Z=v /(v-b)-a / T^{3 / 2} R^{*}(v+b)$

$Z=v /(v-b)-\left[a / T^{3 / 2} R^{*}(v+b)\right]^{*}(b / b)$

$Z=\left\{v /(v-b)-a / b T^{3 / 2} R *[b /(v+b)]\right\} *[(1 / v) /(1 / v)]$

$Z=1 /(1-b / v)-a / b R T^{3 / 2} *[(b / v) /(1+b / v)]$

$h=b / v$

$Z=1 /(1-h)-1 / b R T^{3 / 2} *(h / 1+h)$

$Z=[(1-h)+h] /(1-h)-a / b R T^{3 / 2} *(h / 1+h)$

$Z=1+(h / 1-h)-a / b R T^{3 / 2} *(h / 1+h)$

$Z-1=(h / 1-h)-a / b R T 3 / 2 *(h / 1+h)$

$H^{R} / R T=(Z-1)+T \int_{\infty}^{v}(\partial Z / \partial T)_{v} * d v / v$

$(\partial Z / \partial T)_{v}=\partial / \partial T\left\{v /(v-b)-a / b R T^{3 / 2} *[b /(v+b)]\right\}$

$(\partial Z / \partial T)_{v}=\partial / \partial T\left\{-a / b R T^{3 / 2} *[b /(v+b)]\right\}=d / d T\left\{-a / b R T^{3 / 2} *[b /(v+b)]\right\}$

$(\partial Z / \partial T)_{v}=\left[-a b / b R^{*}(v+b)\right]^{*} d / d T\left(T^{-3 / 2}\right)$

$(\partial Z / \partial T)_{v}=\left[-a b / b R^{*}(v+b)\right]^{*}\left[(-3 / 2) T^{-5 / 2}\right]$

$(\partial Z / \partial T)_{v}=3 a / 2 b R T^{5 / 2} *[b /(v+b)]$

$\int_{\infty}^{v}(\partial Z / \partial T)_{v} * d v / v=\int_{\infty}^{v} 3 a / 2 b R T^{5 / 2} *(b / v+b) * d v / v$

$\int_{\infty}^{v}(\partial Z / \partial T)_{v} * d v / v=3 a / 2 b R T^{5 / 2} \int_{\infty}^{v}(h / 1+h) * d v /(b / h)$

$\int_{\infty}^{v}(\partial Z / \partial T)_{v} * d v / v=3 a / 2 b R T^{5 / 2} \int_{0}^{h}(h / 1+h) * d(b / h) /(b / h)$

$\int_{\infty}^{v}(\partial Z / \partial T)_{v} * d v / v=3 a / 2 b R T^{5 / 2} \int_{0}^{h}(b h / 1+h) * d(1 / h) /(b / h)$

$\int_{\infty}^{v}(\partial Z / \partial T)_{v} * d v / v=3 a / 2 b R T^{5 / 2} \int_{0}^{h}(b h / 1+h)^{*}\left(-1 / h^{2}\right) * d h /(b / h)$

$\int_{\infty}^{v}(\partial Z / \partial T)_{v} * d v / v=3 a / 2 b R T^{5 / 2} \int_{0}^{h}(d h / 1+h) *(-b / h) /(b / h)$ 


$$
\begin{aligned}
& \int_{\infty}^{v}(\partial Z / \partial T)_{v} * d v / v=-3 a / 2 b R T^{5 / 2} \int_{0}^{h}(d h / 1+h) \\
& \int_{\infty}^{v}(\partial Z / \partial T)_{v} * d v / v=-3 a / 2 b R T^{5 / 2} * \ln (1+h)_{0}^{h} \\
& \int_{\infty}^{v}(\partial Z / \partial T)_{v} * d v / v=-3 a / 2 b R T^{5 / 2} *[\ln (1+h)-\ln 1] \\
& \int_{\infty}^{v}(\partial Z / \partial T)_{v} * d v / v=-3 a / 2 b R T^{5 / 2} * \ln (1+h) \\
& H^{R} / R T=(Z-1)+T \int_{\infty}^{v}(\partial Z / \partial T)_{v}^{*} \partial v / v \\
& H^{R} / R T=(Z-1)+T\left(-3 a / 2 b R T^{5 / 2}\right) * \ln (1+h) \\
& H^{R} / R T=(Z-1)-\left(3 a / 2 b R T^{3 / 2}\right)^{*} \ln (1+h) \\
& H^{R} / R T=[h /(1-h)]-a / b R T^{3 / 2} *\{[h /(1+h)]+1.5 \ln (1+h)\} \\
& h=b / v \\
& a=0.42478 R^{2} T_{c}^{2} / P_{c} \\
& b=0.08664 R T_{c} / P_{c}
\end{aligned}
$$




\section{B. Original van der Waals Equation of State}

$$
\begin{aligned}
& P=R T /(v-b)-a / v^{2} \\
& Z=P v / R T=v / R T^{*}\left[R T /(v-b)-a / v^{2}\right] \\
& Z=v /(v-b)-a / v R T \\
& Z=[(v-b)+b] /(v-b)-a / v R T \\
& Z-1=b /(v-b)-a / v R T \\
& H^{R} / R T=(Z-1)+T \int_{\infty}^{v}(\partial Z / \partial T)_{v} * d v / v \\
& (\partial Z / \partial T)_{v}=\partial / \partial T[v /(v-b)-a / v R T] \\
& (\partial Z / \partial T)_{v}=\partial / \partial T(v / v-b)-(a / v R)^{*} \partial / \partial T(1 / T) \\
& (\partial Z / \partial T)_{v}=(-a / v R)^{*} d / d T(1 / T) \\
& (\partial Z / \partial T)_{v}=(-a / v R)^{*}\left(-1 / T^{2}\right) \\
& (\partial Z / \partial T)_{v}=a / v R T^{2} \\
& \int_{\infty}^{v}(\partial Z / \partial T)_{v} * d v / v=\int_{\infty}^{v}\left(a / v R T^{2}\right)^{*} d v / v \\
& \int_{\infty}^{v}(\partial Z / \partial T)_{v}^{*} d v / v=a / R T^{2} \int_{\infty}^{v} d v / v^{2} \\
& \int_{\infty}^{v}(\partial Z / \partial T)_{v}^{*} d v / v=a / R T^{2} *(-1 / v)_{\infty}^{v} \\
& \int_{\infty}^{v}(\partial Z / \partial T)_{v} * d v / v=a / R T^{2} *(-1 / v) \\
& \int_{\infty}^{v}(\partial Z / \partial T)_{v} * d v / v=-a / v R T^{2} \\
& H^{R} / R T=(Z-1)+T \int_{\infty}^{v}(\partial Z / \partial T)_{v} * d v / v \\
& H^{R} / R T=(Z-1)+T\left(-a / v R T^{2}\right) \\
& H^{R} / R T=[b /(v-b)]-(1 / v)^{*}(a / R T)-a / v R T \\
& H^{R} / R T=[b /(v-b)]-2 a / v R T \\
& a=27 R^{2} T_{c}^{2} / 64 P_{c} \\
& b=R T_{c} / 8 P_{c}
\end{aligned}
$$




\section{Modified van der Waals Equation of State}

$$
\begin{aligned}
& Z-1=b /(v-b)-a / v R T \\
& (\partial Z / \partial T)_{v}=\partial / \partial T[b /(v-b)]-\partial / \partial T(a / v R T) \\
& -\partial / \partial T(a / v R T)=\left(-1 / v R^{2}\right)^{*}(d v / d T)^{*}\left(a_{2} / T_{c}-a_{1} / T\right)+a_{1} / v R T^{2} \\
& \partial / \partial T[b /(v-b)]=\left[(v-b)^{*}(d b / d T)-b^{*}(d v / d T)\right] /(v-b)^{2} \\
& d v / d T=0 \\
& (d b / d T)_{v}=(\partial b / \partial v)^{*}(\partial v / \partial T)=0 \\
& d / d T[b /(v-b)]=0 \Rightarrow(\partial z / \partial T)_{v}=a_{1} / v R T^{2} \\
& H^{R} / R T=(Z-1)+T \int_{\infty}^{v}(\partial Z / \partial T)_{v} * d v / v \\
& H^{R} / R T=(Z-1)+T \int_{\infty}^{v}\left(a_{1} / v R T^{2}\right)^{*} d v / v \\
& H^{R} / R T=(Z-1)+\left(a_{1} / R T\right) \int_{\infty}^{v} v^{-2} d v \\
& H^{R} / R T=(Z-1)+\left(a_{1} / R T\right)(-1 / v)_{\infty}^{v} \\
& H^{R} / R T=(Z-1)+\left(a_{1} / R T\right)^{*}(-1 / v-0) \\
& H^{R} / R T=(Z-1)-a_{1} / v R T \\
& H^{R} / R T=b /(v-b)-a_{1} / v R T-a / v R T \\
& H^{R} / R T=b /(v-b)-\left\{\left[a_{1}-a_{2} *\left(T / T_{c}\right)\right] / v R T\right\}-a_{1} / v R T \\
& H^{R} / R T=b /(v-b)-a_{1} / v R T+a_{2}^{*}\left(T / T_{c}\right) / v R T-a_{1} / v R T \\
& H^{R} / R T=b /(v-b)-2 a_{1} / v R T+a_{2} *\left(T / T_{c}\right) / v R T \\
& H^{R} / R T=b /(v-b)-2 a_{1} / v R T+a_{2} / v R T_{c}
\end{aligned}
$$




\section{VITA}

Andrew James Sexton was born on May 20, 1981, to Joe and Carol Sexton in Somerset, Kentucky. He grew up in nearby Ferguson, Kentucky, along with his sister Megan (21) and brothers Matthew (26) and Samuel (18).

Andrew graduated from Southwestern High School, located in Somerset, in 1999 and joined his brother Matthew at the University of Louisville. After completing a B.S.Ch.E., he pursued the Master of Engineering. Upon completing the M. Eng., Andrew plans to attend the University of Texas at Austin and pursue a Ph.D. 